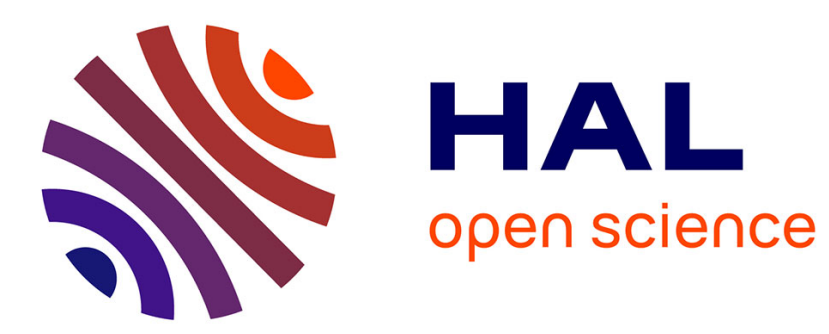

\title{
Introduction à la relaxation électronique dans les solides : mécanismes et techniques de mesure
}

P. Bonville

\section{To cite this version:}

P. Bonville. Introduction à la relaxation électronique dans les solides: mécanismes et techniques de mesure. Revue de Physique Appliquée, 1983, 18 (7), pp.365-411. 10.1051/rphysap:01983001807036500 . jpa-00245104

HAL Id: jpa-00245104

https://hal.science/jpa-00245104

Submitted on 1 Jan 1983

HAL is a multi-disciplinary open access archive for the deposit and dissemination of scientific research documents, whether they are published or not. The documents may come from teaching and research institutions in France or abroad, or from public or private research centers.
L'archive ouverte pluridisciplinaire HAL, est destinée au dépôt et à la diffusion de documents scientifiques de niveau recherche, publiés ou non, émanant des établissements d'enseignement et de recherche français ou étrangers, des laboratoires publics ou privés. 


\title{
Introduction à la relaxation électronique dans les solides : mécanismes et techniques de mesure
}

\author{
P. Bonville \\ SPSRM, CEN-Saclay, 91191 Gif-sur-Yvette Cedex, France
}

(Reçu le 23 février 1983, accepté le 24 mars 1983)

\begin{abstract}
Résumé. - Les fluctuations des moments magnétiques électroniques dans les solides peuvent être étudiées par plusieurs techniques, électroniques ou nucléaires. Cet article est une introduction aux mécanismes de relaxation paramagnétique les plus fréquemment rencontrés (phonons, électrons de conduction, interactions d'échange ou dipolaires) dans la matière condensée, et aux différentes techniques de mesure des fréquences de relaxation : résonance paramagnétique électronique, résonance nucléaire, spectroscopie Mössbauer, diffusion inélastique des neutrons, mesures de susceptibilité alternative longitudinale et corrélations angulaires perturbées $\gamma-\gamma$. Nous portons principalement notre attention dans cet article sur les spectres des fluctuations individuelles des ions, et les exemples sont extraits en majorité de travaux expérimentaux sur les terres rares.
\end{abstract}

\begin{abstract}
The fluctuations of electronic magnetic moments in solids may be investigated by several techniques, either electronic or nuclear. This paper is an introduction to the most frequently encountered paramagnetic relaxation mechanisms (phonons, conduction electrons, exchange or dipolar interactions) in condensed matter, and to the different techniques used for measuring relaxation frequencies : electronic paramagnetic resonance, nuclear magnetic resonance, Mössbauer spectroscopy, inelastic neutron scattering, measurement of longitudinal ac susceptibility and $\gamma-\gamma$ perturbed angular correlations. We mainly focus our attention on individual ionic fluctuation spectra, the majority of the experimental work refered to concerning rare earth systems.
\end{abstract}

\section{Introduction.}

Considérons un solide contenant des impuretés paramagnétiques d'ions de transition ou de terre rare. Lorsque l'ensemble est en équilibre thermique à la température $T$, le couplage existant entre le moment magnétique de l'ion d'une part, et les vibrations du réseau ou les électrons de conduction (dans le cas d'un métal) d'autre part, induit entre ces deux systèmes des échanges d'énergie. Ceux-ci peuvent également être considérés comme des fluctuations temporelles du moment électronique et de son environnement.

Ces échanges d'énergie maintiennent l'équilibre de Boltzmann à la température $T:$ si $|a\rangle$ et $|b\rangle$ sont deux états électroniques de l'impureté, séparés par une énergie : $\hbar \omega_{a b}=E_{b}-E_{a}>0$, les probabilités $W_{a \rightarrow b}$ et $W_{b \rightarrow a}$ de transition $\left({ }^{1}\right)$ entre les deux

( $\left.{ }^{1}\right)$ Note sur les unités : Dans cet article, les probabilités de transition, homogènes à $\left[\mathrm{s}^{-1}\right]$, sont exprimées en unités de fréquence $(\mathrm{Hz})$. Pour obtenir les valeurs en $\mathrm{s}^{-1}$, il convient de multiplier par $2 \pi(\omega=2 \pi v)$. états, accompagnée respectivement d'absorption et d'émission de l'énergie $\hbar \omega_{a b}$, vérifient le principe de "l'équilibre microscopique " :

$$
W_{a \rightarrow b}(T) / W_{b \rightarrow a}(T)=\mathrm{e}^{-\hbar \omega_{a b} / k_{B} T} .
$$

Lorsque la température est élevée par rapport à l'écart en énergie entre les deux niveaux, les transitions d'absorption et d'émission deviennent équiprobables, et nous les désignerons par $W(T)$.

Lorsque la concentration en ions magnétiques augmente, de manière que les interactions d'échange ou dipolaires magnétiques deviennent importantes, des fluctuations ont lieu également au sein du système de moments, induites par ces interactions "spinspin ".

Il est possible de porter artificiellement le système électronique (nous parlerons également de système électronucléaire, en vue des applications aux techniques hyperfines d'observation) hors équilibre, par pompage optique ou de radiofréquence, par renversement rapide d'un champ magnétique polarisant, ou bien à la suite d'une cascade nucléaire radioactive (dans le cas de niveaux hyperfins ou électronucléaires). 
La « thermalisation », ou retour vers l'état d'équilibre, s'effectue alors en un temps de l'ordre de $1 / W(T)$ après l'application de la perturbation $\left({ }^{2}\right)$. Le terme « relaxation » possède usuellement ce sens de retour vers l'équilibre.

Plus généralement, l'étude de la relaxation électronique (ou paramagnétique) est l'étude des transitions entre les niveaux quantiques des ions dans les solides induites par l'interaction entre le moment magnétique électronique et son environnement.

Les probabilités de transition électronique $W_{a \rightarrow b}$ sont couramment appelées «fréquences de relaxation $"$.

Les moments magnétiques des noyaux interagissent également avec leur environnement. On parle alors de relaxation nucléaire.

Les techniques d'observation des phénomènes de relaxation dans les corps solides sont assez diverses, et dans leur ensemble, couvrent un domaine de fréquences de relaxation allant de quelques $\mathrm{Hz}$ à quelque $10^{15} \mathrm{~Hz}$, sur un domaine de température compris entre quelques centièmes de $\mathrm{K}$ et $1000 \mathrm{~K}$. Elles peuvent être classifiées en deux catégories : les techniques réalisant une mesure directe sur le système électronique et celles utilisant un couplage avec une variable nucléaire, le système électronique étant alors « perçu » par l'intermédiaire de l'interaction hyperfine.

Parmi les mesures "directes", on peut ranger la mesure de la susceptibilité alternative longitudinale $\chi_{\|}(\omega)$, la résonance paramagnétique électronique (R.P.E.) (ces deux techniques nécessitant l'application d'un champ magnétique), la spectroscopie optique, et la diffusion inélastique des neutrons (D.I.N.).

Les techniques « hyperfines " comptent la spectroscopie Mössbauer de rayons $\gamma$, les mesures de corrélations angulaires perturbées $\gamma-\gamma$ différentielles ou intégrales (C.A.P.), et la résonance magnétique nucléaire (R.M.N.). Ces deux dernières techniques permettent en fait de mesurer directement les temps de relaxation nucléaire, et donnent accès aux fréquences électroniques dans les alliages dilués en ions magnétiques. En effet, dans ce cas, l'interaction hyperfine constitue le mécanisme dominant de relaxation pour le noyau de l'ion ou pour un noyau proche voisin de l'impureté.

Certaines de ces techniques, comme la R.M.N. [1], les mesures optiques [2], la diffusion des neutrons [3], les C.A.P. [4] ont été largement appliquées à l'étude de la relaxation des molécules dans les liquides et les gaz.

$\left({ }^{2}\right)$ Nous excluons de notre étude le phénomène de retour vers l'équilibre de l'aimantation transversale $\mathbf{M}_{x}$ en R.P.E. ou R.M.N. après une " impulsion à $90^{\circ}$ ", lorsque la décroissance de $M_{x}$ est due à la perte de la cohérence de phase des différents paquets de spins causée par les inhomogénéités du champ dipolaire local (cf. $\S 4.2 .3$ sur la formation des " échos de spins "),
Les méthodes de mesure utilisées peuvent être classifiées selon le mode d'analyse du signal observé : en fonction du temps ou de l'énergie.

Dans les méthodes d'analyse en temps du signal, on observe l'évolution temporelle d'une grandeur caractéristique du système électronique (ou nucléaire) comme l'aimantation ou la fluorescence, après l'application d'une perturbation intense et brève qui amène ce système dans un état hors équilibre thermique. Dans de nombreux cas, cette évolution obéit à une loi exponentielle $\mathrm{e}^{-W t}$, et l'équilibre est atteint au bout d'un temps de l'ordre de $1 / W$.

Les méthodes d'analyse en énergie consistent à observer, en régime continu, la réponse en fréquence du système électronique à une excitation de faible intensité qui perturbe très peu l'équilibre de Boltzmann. Par exemple, les techniques résonnantes électromagnétiques utilisant l'analyse en énergie (effet Mössbauer, R.P.E., R.M.N.) effectuent un «balayage » autour de l'énergie de résonance (qui correspond à une énergie de transition dans le système électronique ou nucléaire). On mesure un «spectre" de raies d'absorption ou d'émission d'énergie en fonction de la fréquence. Les niveaux du système atomique, acquérant un temps de vie $1 / W$ sous l'effet des transitions liées aux fluctuations de fréquence $W$, confèrent à la raie spectroscopique un élargissement $W$, conformément au principe d'incertitude de Heisenberg. En fait, dans certains cas, la présence d'une structure dans le spectre observé rend cette image simple inadéquate, et c'est la forme globale du spectre qui dépend de la fréquence $W$.

La mesure de la constante de temps caractérisant le retour du signal vers sa valeur d'équilibre dans le premier type de méthode, et l'examen de la forme de raie dans le second type donnent directement accès au paramètre dynamique $W(T)$, qui dépend des constantes de couplage du système électronique à son environnement, et qui est en général une fonction croissante de la température.

La dépendance thermique de la fréquence de relaxation constitue en général une "signature " caractéristique d'un mécanisme particulier de relaxation, et sa détermination est la base de toute interprétation ultérieure des mesures pour extraire l'information physique.

La plupart des études de relaxation électroniques sont réalisées à basse température $(T \lesssim 100 \mathrm{~K})$, pour deux raisons principales :

i) Seul un petit nombre de niveaux électroniques est appréciablement peuplé lorsque la température est faible devant l'énergie de champ cristallin ou du couplage spin-orbite, ce qui simplifie grandement l'extraction des paramètres physiques. Un cas fréquemment rencontré est celui d'un doublet de champ cristallin d'un ion de Kramers, caractérisé par un spin effectif $S=1 / 2$ et un tenseur spectroscopique $\mathbf{g}$, tel que la projection du moment total $g_{J} \mu_{\mathrm{B}} J$ de l'ion de terre rare sur ce doublet soit : $\mathbf{g} \mu_{\mathrm{B}} \mathbf{S}$. 
Tableau I. - Caractéristiques expérimentales des mesures de relaxation électronique par diverses techniques.

\begin{tabular}{|c|c|c|c|c|c|}
\hline $\begin{array}{l}\text { Technique de } \\
\text { mesure }\end{array}$ & $\begin{array}{l}\text { Type de } \\
\text { mesure }\end{array}$ & $\begin{array}{l}\text { Méthode } \\
\text { d'analyse } \\
\text { du signal }\end{array}$ & $\begin{array}{c}\text { Domaine } \\
\text { expérimental } \\
\text { de mesure de } W_{\mathbf{R}}\end{array}$ & $\begin{array}{l}\text { Domaine de } \\
\text { température }\end{array}$ & Domaine d'applications \\
\hline$\chi_{\|}(\omega)$ & directe & énergie & $1-10^{8} \mathrm{~Hz}$ & $T<10 \mathrm{~K}$ & $\begin{array}{l}\text { Relaxation spin-spin et spin-réseau } \\
\text { dans les composés isolants. }\end{array}$ \\
\hline \multirow{2}{*}{$\begin{array}{l}\text { Résonance } \\
\text { paramagnétique } \\
\text { électronique }\end{array}$} & \multirow{2}{*}{ directe } & temps & $1-10^{6} \mathrm{~Hz}$ & $T<4 \mathrm{~K}$ & $\begin{array}{l}\text { Analyse en temps : relaxation spin- } \\
\text { réseau d'impuretés dans une } \\
\text { matrice isolante. }\end{array}$ \\
\hline & & énergie & $10^{7}-10^{9} \mathrm{~Hz}$ & \begin{tabular}{|c|}
$T<20 \mathrm{~K}$ \\
sauf pour les ions \\
$\mathrm{S}\left(\mathrm{Gd}^{3+}\right):$ \\
$T \lesssim 300 \mathrm{~K}$
\end{tabular} & $\begin{array}{l}\text { Analyse en énergie : relaxation par } \\
\text { les électrons de conduction d'im- } \\
\text { puretés dans les matrices métal- } \\
\text { liques. }\end{array}$ \\
\hline $\begin{array}{l}\text { Diffusion } \\
\text { inélastique des } \\
\text { neutrons }\end{array}$ & directe & énergie & $10^{10}-10^{14} \mathrm{~Hz}$ & $T<300 \mathrm{~K}$ & $\begin{array}{l}\text { Composés intermétalliques de } \\
\text { terre rare. }\end{array}$ \\
\hline $\begin{array}{l}\text { Spectroscopie } \\
\text { Mössbauer }\end{array}$ & hyperfine & $\begin{array}{l}\text { énergie et } \\
\text { temps }\left({ }^{++}\right)\end{array}$ & $10^{6}-10^{11} \mathrm{~Hz}\left(^{*}\right)$ & $T<300 \mathrm{~K}\left({ }^{*}\right)$ & $\begin{array}{l}\text { Très variés : composés isolants } \\
\text { ou métalliques, dilués ou con- } \\
\text { centrés en ions magnétiques. }\end{array}$ \\
\hline $\begin{array}{l}\text { Corrélations } \\
\text { angulaires } \\
\text { perturbées }\end{array}$ & hyperfine & temps $\left({ }^{* *}\right)$ & & $T>300 \mathrm{~K}$ & $\begin{array}{l}\text { Relaxation par les électrons de } \\
\text { conduction d'impuretés, dans } \\
\text { les matrices métalliques. }\end{array}$ \\
\hline $\begin{array}{l}\text { Résonance } \\
\text { magnétique } \\
\text { nucléaire }\end{array}$ & hyperfine & $\begin{array}{l}\text { énergie } \\
\text { et } \\
\text { temps }\end{array}$ & & & \\
\hline
\end{tabular}

$\left({ }^{*}\right)$ Variable selon l'isotope utilisé.

$\left(^{+}\right)$Il s'agit de la fréquence de relaxation électronique. La fréquence de relaxation du noyau de l'impureté (ou proche voisin de l'impureté) est dans ce cas inférieure à $1 \mathrm{MHz}$.

$\left({ }^{++}\right)$Dans certains cas, en spectrométrie d'émission, avec ou sans coïncidences $\gamma-\gamma$, lorsque $W_{\mathbf{R}} \sim 1 / \tau_{\mathrm{n}}\left(\tau_{\mathrm{n}}\right.$ : temps de vie du niveau nucléaire excité).

$\left({ }^{* *}\right)$ Les corrélations angulaires intégrales (sans coïncidences $\gamma-\gamma$ ) permettent de mesurer $W_{\mathrm{R}}$ lorsque : $W_{\mathrm{R}} \sim 1 / \tau_{\mathrm{n}}$ $\left(\tau_{n}:\right.$ temps de vie du niveau nucléaire intermédiaire).

ii) Suivant les caractéristiques du corps étudié (métal, isolant, ions magnétiques dilués ou concentrés), il y a prédominance à basse température d'un seul mécanisme de relaxation, ce qui rend sa caractérisation par la variation thermique de la fréquence $W(T)$ associée plus aisée.

Pour fixer les idées, on peut dire que les fréquences de relaxation électroniques dans les solides sont inférieures à $10^{11} \mathrm{~Hz}$ dans la région $T \leqslant 50 \mathrm{~K}$.

Certaines techniques (D.I.N., C.A.P., R.M.N.) permettent cependant d'avoir accès à des fréquences de relaxation électronique jusqu'à $10^{14} \mathrm{~Hz}$; leur application à plus haute température (jusqu'à température ambiante et même jusqu'à $1000 \mathrm{~K}$ en C.A.P.) s'est beaucoup développée depuis une dizaine d'années, surtout dans les alliages dilués, en relation avec le problème de l'effet Kondo et des instabilités de valence des ions de terre rare.
Le tableau I résume certaines caractéristiques des techniques de mesure évoquées jusqu'ici, à l'exception des mesures optiques, dont nous parlerons peu. La dernière colonne du tableau concerne le type de composés et de mécanismes de relaxation qui constituent le champ d'application priviligié d'une technique donnée.

Cet exposé, qui veut être une introduction à l'étude des phénomènes de relaxation à l'aide des techniques spectroscopiques utilisées en physique du solide, ne prétend pas constituer une revue exhaustive du sujet concerné. On a tenté plutôt de mettre en évidence les concepts physiques communs sous-jacents à la mesure des temps et fréquences de relaxation électronique par les diverses techniques ; la description des processus d'échange d'énergie du système électronique avec son environnement est illustrée principalement par des exemples empruntés aux travaux 
expérimentaux sur les ions de terre rare, et occasionnellement sur les ions de transition.

L'organisation de cet article est détaillée dans ce qui suit. Le paragraphe 2 introduit une approche perturbative de la relaxation et schématise le calcul de l'évolution temporelle de la matrice densité du système électronique, avant d'examiner si les différents mécanismes de relaxation sont justiciables d'un traitement en perturbation. Le paragraphe 3 contient une description des deux méthodes d'analyse en temps et en énergie et indique le principe du calcul des signaux observés, l'accent étant mis sur l'analyse en énergie du spectre. Dans le paragraphe 4 sont décrites les techniques de mesure, ainsi qu'un effet commun à la spectroscopie Mössbauer, la R.P.E. et la spectroscopie neutronique : «l'effacement» de la structure des spectres en énergie par la relaxation. Enfin les deux derniers paragraphes traitent de l'information physique recueillie à partir des études de relaxation électronique. Le paragraphe 5 concerne les dépendances thermiques de $W(T)$ dans le cas de la relaxation spin-phonons et par les électrons de conduction. Dans le paragraphe 6 , qui traite de la relaxation spin-spin nous avons insisté sur les différences importantes entre les signaux mesurés par R.P.E. et effet Mössbauer dans les corps à forte concentration d'ions magnétiques.

\section{Description perturbative de la relaxation.}

Dans de nombreux cas expérimentaux, le système électronique est «faiblement couplé » à son environnement (appelé encore «bain»), en particulier lorsqu'il consiste en une impureté dans une matrice hôte.

Le hamiltonien du solide que nous considérerons s'écrit :

$$
H=\mathscr{H}_{0}+\mathscr{H}_{1}+\mathscr{H}_{\mathbf{b}},\left[\mathscr{H}_{\mathbf{b}}, \mathscr{H}_{0}\right]=0,
$$

où $\mathscr{H}_{0}$ représente le hamiltonien définissant les états quantiques du système atomique (champ cristallin, énergie Zeeman, énergie hyperfine), $H_{b}$ les degrés de liberté du bain, caractéristique de la matrice (vibrations du réseau, électrons de conduction) et $H_{1}$ l'interaction entre le système électronique et le bain. On considère souvent pour ce dernier la forme :

$$
\mathscr{H}_{1}=A \mathrm{~S} . \mathrm{B} \text {, }
$$

dans laquelle $A$ représente la force de l'interaction, $S$ est un opérateur décrivant l'ion (en général le spin ou le moment orbital) et $B$ est un opérateur représentant la variable du bain couplée au système électronique.

Dans un traitement de perturbation, l'interaction $\mathscr{H}_{1}$ induit des transitions entre les niveaux du système atomique. Nous allons considérer par exemple une transition entre deux états électroniques $|a\rangle$ et $|b\rangle$.
Dans une approche semi-classique du problème, on calcule la probabilité de transition au sein du système $\mathscr{H}_{0}$ induite par l'interaction $\mathscr{H}_{1}(t)=A \mathbf{S} . \mathbf{B}(t)$, où l'on considère que l'opérateur $\mathbf{B}(t)$ dépend explicitement $\mathrm{du}$ temps et fluctue de manière aléatoire. On obtient [1] :

$$
\begin{aligned}
W_{a \rightarrow b}=\frac{A^{2}}{\hbar^{2}}\left|\left\langle b\left|S_{-}\right| a\right\rangle\right|^{2} \times & \\
& \times \int_{-\infty}^{+\infty} \mathrm{d} \tau \mathrm{e}^{i \omega_{a b} \tau}\left\langle B^{+}(\tau) \cdot B^{-}(0)\right\rangle
\end{aligned}
$$

en supposant que $|a\rangle$ et $|b\rangle$ sont états propres de la composante $S_{z}$ du spin de l'ion. La quantité $\left\langle B^{+}(\tau) \cdot B^{-}(0)\right\rangle$ est la fonction d'autocorrélation de l'opérateur $B$, le signe $\langle>$ désignant une moyenne prise sur les fluctuations aléatoires.

Phénoménologiquement, on considère souvent une dépendance exponentielle pour la fonction de corrélation, avec un temps caractéristique $\tau_{\mathbf{c}}$ :

$$
\left\langle B^{+}(\tau) B^{-}(0)\right\rangle=\left\langle B^{2}\right\rangle \mathrm{e}^{-|\tau| / \tau_{c}} .
$$

$\tau_{\mathrm{c}}$ est une mesure de la "mémoire " des fluctuations dans le bain. La probabilité de transition $W_{a \rightarrow b}$ fait intervenir la « densité spectrale » des fluctuations du bain :

$$
J(\omega)=\int_{-\infty}^{+\infty} \mathrm{d} \tau \mathrm{e}^{i \omega \tau}\left\langle B^{+}(\tau) \cdot B^{-}(0)\right\rangle
$$

et :

$$
W_{a \rightarrow b}=\frac{A^{2}}{\hbar^{2}}\left|\left\langle b\left|S^{+}\right| a\right\rangle\right|^{2} J\left(\omega_{a b}\right)
$$

$J\left(\omega_{a b}\right)$ est la composante de Fourier à l'énergie de la transition $\hbar \omega_{a b}$ de la fonction de corrélation de l'opérateur $\mathbf{B}(t)$ du bain. Cette densité spectrale mesure la probabilité que le bain cède ou reçoive l'énergie $\hbar \omega_{a b}$.

Dans l'hypothèse (4) d'une décroissance exponentielle de la fonction de corrélation, la densité spectrale $J(\omega)$ est une lorentzienne de demi-largeur à mihauteur $1 / \tau_{\mathrm{c}}$ :

$$
J(\omega)=\left\langle B^{2}\right\rangle \frac{2 \tau_{c}}{1+\omega^{2} \tau_{c}^{2}} .
$$

Dans le cas où l'énergie de transition $\hbar \omega_{a b}$ est bien inférieure à $\hbar / \tau_{c}$, c'est-à-dire lorsque :

$$
\omega_{a b} \tau_{\mathrm{c}} \ll 1,
$$

la densité spectrale $J\left(\omega_{a b}\right)$ devient indépendante de la fréquence $\omega_{a b}$ :

$$
J\left(\omega_{a b}\right) \simeq 2\left\langle B^{2}\right\rangle \tau_{c} .
$$

Cette situation est appelée « approximation du spectre blanc » (ou W.N.A. : " White Noise Approximation »). Le calcul semi-classique des probabilités de transi- 
tion $W_{a \rightarrow b}$ n'est cependant pas suffisant pour décrire la réalité expérimentale pour deux raisons :

i) la seule considération des probabilités de transition suppose que le système électronique est décrit complètement par les populations des niveaux d'énergie, c'est-à-dire par les seuls éléments diagonaux de sa matrice densité $\sigma$. Or, nous verrons que les mesures par les techniques spectroscopiques mettent souvent en jeu les éléments non diagonaux de $\sigma$ (R.P.E. et R.M.N. après une "impulsion à $90^{\circ}$ ", effet Mössbauer, ...).

ii) Il est facile de se convaincre que l'approche semiclassique n'est valable qu'à haute température $\left(\hbar \omega_{a b} \ll k_{\mathrm{B}} T\right)$. En effet, la relation (5) montre que :

$$
\frac{W_{a \rightarrow b}}{W_{b \rightarrow a}}=\frac{J\left(\omega_{a b}\right)}{J\left(\omega_{b a}\right)},
$$

et l'expression (6) de $J(\omega)$ implique que ce rapport vaut 1 , en contradiction avec le principe de "l'équilibre microscopique " (relation (1)). A haute température, cependant, la relation (1) donne :

$$
W_{a \rightarrow b}=W_{b \rightarrow a} .
$$

Expérimentalement, on rencontre de nombreuses situations "basse température " : en spectroscopie Mössbauer, lorsque la température devient comparable aux énergies hyperfines $(T \sim 0,1 \mathrm{~K})$, ou en spectroscopie de neutrons inélastiques, lorsque : $k_{\mathrm{B}} T \sim \Delta_{\mathrm{cc}}$, où $\Delta_{\mathrm{cc}}$ est une énergie de champ cristallin.

Une approche correcte consiste donc à considérer l'évolution temporelle de la matrice densité totale $\sigma$ $d u$ système atomique, qui, comme nous le verrons (\$3), permet de déterminer les signaux mesurés à l'aide des différentes techniques. De plus, il convient dans la mesure du possible, de tenir compte du caractère quantique du bain dans l'évaluation des densités spectrales.

Rappelons que la matrice densité du système atomique à l'équilibre de Boltzmann à température $T$ vaut :

$$
\sigma_{\mathbf{B}}=\mathrm{e}^{-\mathcal{E}_{0} / k_{\mathbf{B}} T} / \operatorname{Tr}\left(\mathrm{e}^{-\varkappa_{0} / k_{\mathbf{B}} T}\right) .
$$

En présence du hamiltonien du bain $\mathcal{H}_{\mathrm{b}}$ et du couplage de relaxation $\mathcal{H}_{1}$, l'évolution temporelle de la matrice densité $D$ totale du solide s'écrit :

$$
\frac{\mathrm{d} D}{\mathrm{~d} t}=i\left[D, \mathcal{H}_{0}+\mathcal{H}_{1}+\mathcal{H}_{b}\right] .
$$

La matrice densité $\sigma$ du système électronique (ou électronucléaire) étant donnée par :

$$
\sigma=\operatorname{Tr}_{\text {bain }}(D),
$$

son équation d'évolution s'écrit, d'après (10) :

$$
\frac{\mathrm{d} \sigma}{\mathrm{d} t}=i \operatorname{Tr}_{\mathrm{bain}}[D, \mathcal{H}] \text {. }
$$

Une approximation de perturbation consiste alors à intégrer cette équation par itération jusqu'au $2^{\mathrm{e}}$ ordre en $H_{1}$, puis à effectuer les moyennes sur le bain en supposant qu'à chaque instant ce dernier reste en équilibre thermique $[1,5]$ :

$$
D(t)=\sigma(t) \rho_{\mathrm{b}},
$$

où

$$
\rho_{\mathrm{b}}=\mathrm{e}^{-\mathfrak{H}_{\mathrm{b}} / k_{\mathrm{B}} T} / \operatorname{Tr}\left(\mathrm{e}^{-\mathfrak{H}_{\mathrm{b}} / k_{\mathrm{B}} T}\right) .
$$

On obtient alors une équation d'évolution linéaire pour $\sigma(t)$ :

$$
\frac{\mathrm{d} \sigma}{\mathrm{d} t}=-i\left[\mathfrak{H}_{0}, \sigma\right]+S \sigma
$$

La matrice $S$ est une matrice à 4 indices : $\left\{S_{a a^{\prime}, b b^{\prime}}\right\}$. $\mathrm{Si}|a\rangle,\left|a^{\prime}\right\rangle,|b\rangle,\left|b^{\prime}\right\rangle$ sont des états propres de $\mathcal{J}_{0}$ :

$$
\frac{\mathrm{d} \sigma_{a a^{\prime}}}{\mathrm{d} t}=i \omega_{a a^{\prime}} \sigma_{a a^{\prime}}+\sum_{b, b^{\prime}} S_{a a^{\prime}, b b^{\prime}} \sigma_{b b^{\prime}} .
$$

Les éléments de matrice $S_{a a^{\prime}, b b^{\prime}}$ sont des combinaisons linéaires des densités spectrales du bain aux fréquences propres du système atomique, et ils sont de l'ordre de grandeur des fréquences de relaxation $W_{a \rightarrow b}$. Une expression complète de $S_{a a^{\prime}, b b^{\prime}}$ valable à toute température, est donnée dans la référence [6]. Certains éléments ont une interprétation physique simple. Par exemple : $S_{a a, b b}=-2 W_{a \rightarrow b}$.

Les densités spectrales $J(\omega)$ peuvent être calculées quantiquement dans certains cas, par exemple lorsque la relaxation du spin électronique est due au couplage avec les phonons ou les électrons de conduction (cf. § 5). Nous verrons qu'elles s'éloignent considérablement de la forme lorentzienne obtenue dans le cas semi-classique (expression (6)), ce qui montre que l'hypothèse d'une décroissance exponentielle de la fonction de corrélation du bain ne correspond pas à la réalité dans les solides. De plus, les densités spectrales ainsi calculées vérifient le principe du bilan élémentaire, comme on peut le voir en combinant (1) et (5) :

$$
J\left(\omega_{a b}\right) / J\left(\omega_{b a}\right)=\mathrm{e}^{-\eta \omega_{a b} / k_{B} T} .
$$

Lorsque l'interaction de relaxation dominante est l'échange avec les autres moments magnétiques localisés $\left({ }^{3}\right)$, le calcul quantique est en général irréalisable et on utilise en première approximation (cf. § 6)

$\left({ }^{3}\right)$ Signalons que, dans le cas de la R.P.E. dans un système concentré en ions magnétiques identiques, l'interaction d'échange isotrope ne contribue pas à la relaxation « longitudinale ». Par contre, si l'on observe les fluctuations des ions individuels (effet Mössbauer, ou R.P.E. d'une impureté couplée par échange à d'autres moments magnétiques) l'interaction d'échange isotrope est un processus de relaxation efficace (cf. § 6). 
une forme gaussienne pour la fonction de corrélation des fluctuations de la matrice d'ions en interaction :

$$
\left\langle B^{+}(\tau) B(0)\right\rangle=\left\langle B^{2}\right\rangle \mathrm{e}^{-\Delta^{2} \tau^{2}} .
$$

La densité spectrale $J(\omega)$ est alors également une gaussienne, de demi-largeur $\Delta$.

Examinons maintenant quelles sont les hypothèses contenues dans le calcul que nous venons d'esquisser.

La condition pour que le développement de perturbation aboutissant à l'équation (14) converge s'écrit [5] :

$$
S_{a a^{\prime}, b b^{\prime}} \sim W \ll 1 / \tau_{c} \text {. }
$$

En d'autres termes, pour que l'interaction donnant lieu à la relaxation se prête à une description perturbative, il faut que la fréquence des fluctuations induites sur le système électronique soit bien inférieure à la largeur des densités spectrales du bain.

Nous avons également supposé que le bain luimême n'est pas perturbé par la présence du système électronique et qu'il reste à tout instant en équilibre thermique.

Cette condition est en général vérifiée, sauf dans le cas, rencontré dans les expériences de R.P.E., où il se produit un " goulot d'étranglement " (bottleneck) au niveau du transfert d'énergie du bain de phonons ou d'électrons de conduction vers le « bain principal » (en général le liquide réfrigérant). Ce phénomène est dû au couplage du système électronique avec des phonons " résonnants " [7] ou avec les électrons de conduction du métal lorsque le facteur spectroscopique $g$ de l'ion est égal à celui des électrons itinérants $(g=2)[8]$. En présence de "bottleneck », le signal observé correspond à l'évolution temporelle du système couplé ion-bain "résonnant ", et n'est donc plus caractéristique du système électronique seul. Dans la suite, nous ferons abstraction de ce phénomène, et considérerons que le système électronique ne " chauffe " pas le réseau.

Nous allons maintenant examiner si les différents mécanismes de relaxation électronique à l'œuvre dans les solides sont susceptibles d'être traités en perturbation au $2^{e}$ ordre, c'est-à-dire si : $W \ll 1 / \tau_{\mathrm{c}}$

2.1 Relaxation PAR LeS PHONONS. - Le moment orbital électronique de l'ion interagit avec les vibrations du réseau cristallin (cf. $§ 5$ ); les transitions entre niveaux électroniques sont accompagnées d'absorption et/ou d'émission d'un ou plusieurs phonons. Les impuretés magnétiques dans les solides isolants relaxent par ces processus, appelés souvent « relaxation spin-réseau ").

Le temps de corrélation associé aux phonons peut être estimé à l'aide de la largeur de la densité spectrale des phonons acoustiques (cf. $\S 5$ ) :

$$
\frac{1}{\tau_{\mathrm{c}}} \sim \Delta \omega \sim \frac{k_{\mathrm{B}} \theta_{\mathrm{D}}}{\hbar},
$$

où $\theta_{\mathrm{D}}$ est la température de Debye du corps.
Les valeurs usuelles de $\theta_{\mathrm{D}}$ (quelque $100 \mathrm{~K}$ ) conduisent à des temps de corrélation de l'ordre de $\tau_{\mathrm{c}} \simeq 10^{-13} \mathrm{~s}$.

Les fréquences de relaxation associées à ces processus ont été mesurées dans un grand nombre d'expériences, surtout par R.P.E. (voir par exemple la Réf. [9]). A basse température (1 $\mathrm{K}<T<50 \mathrm{~K})$, elles sont comprises approximativement entre $100 \mathrm{~Hz}$ et $10^{9} \mathrm{~Hz}$ et sont donc bien inférieures à :

$$
\frac{1}{\tau_{\mathrm{c}}} \sim 10^{13} \mathrm{~Hz},
$$

ce qui justifie a posteriori le traitement en perturbation usuellement appliqué aux mécanismes de relaxation « spin-réseau ».

2.2 RelaXATION PAR LES ÉLECTRONS DE CONDUCTION. - Pour les impuretés magnétiques dans les substances métalliques, le mécanisme principal de relaxation est l'échange entre le spin du moment localisé $\mathbf{S}$ et le spin des électrons itinérants $\mathbf{s}$ :

$$
H_{1}=-2 J_{\mathrm{ex}} \mathrm{sS},
$$

où $J_{\text {ex }}$ est la constante d'échange.

Nous verrons au paragraphe 5 que la largeur de la densité spectrale associée à la diffusion des électrons de conduction, à température $T$, est de l'ordre de : $\Delta \omega \sim k_{\mathrm{B}} T / \hbar$. La statistique de Fermi, à laquelle obéissent les électrons du métal, impose en effet que seule une bande d'énergie de largeur $k_{\mathrm{B}} T$ de part et d'autre de l'énergie de Fermi soit disponible pour les excitations des électrons de la bande de conduction. A haute température $\left(\hbar \omega_{a b} \ll k_{\mathrm{B}} T\right)$, la densité spectrale à la fréquence $\omega_{a b}$ vaut approximativement (cf. § 5) :

$$
J\left(\omega_{a b}\right) \sim \hbar n^{2}\left(E_{\mathrm{F}}\right) k_{\mathrm{B}} T,
$$

où $n\left(E_{\mathrm{F}}\right)$ est la densité d'états des électrons de conduction au niveau de Fermi. D'après la relation (5), la fréquence de relaxation électronique est alors une fonction linéaire de la température (loi de Korringa) :

$$
W^{\text {E.C. }} \simeq \frac{1}{\hbar} J_{\mathrm{ex}}^{2} n^{2}\left(E_{\mathrm{F}}\right) k_{\mathrm{B}} T \text {. }
$$

La condition (17) s'écrit : $W^{\text {E.c. }} \ll \Delta \omega$, c'est-à-dire :

$$
\left|J_{\text {ex }} n\left(E_{\mathrm{F}}\right)\right| \ll 1 .
$$

De nombreuses expériences [10] ont montré que cette condition est remplie pour des ions de terre rare interagissant par échange atomique avec les électrons de conduction, où typiquement :

$$
J_{\mathrm{ex}}^{\text {at }} \sim 0,05 \mathrm{eV} \text { et } n\left(E_{\mathrm{F}}\right) \sim 0,1-1 \mathrm{eV}^{-1} \mathrm{at}^{-1} .
$$

On peut alors se limiter au second ordre en perturbation. Tel n'est pas le cas en présence d'effet Kondo [11] sur les ions de transition $3 \mathrm{~d}$ ou les terres rares $\mathrm{Yb}^{3+}$ et $\mathrm{Ce}^{3+}$; la constante d'échange effective 
$J_{\text {ex }}^{\text {eff }}$ (négative) vaut alors environ $-0,5 \mathrm{eV}$, et le paramètre $\left|J_{\text {ex }}^{\text {eff }}\right| n\left(E_{\mathrm{F}}\right)$ prend des valeurs $(0,1-0,2)$ telles que le développement en perturbation doit être poussé jusqu'au $3^{\mathrm{e}}$ ordre en $J_{\mathrm{ex}}^{\text {eff }} n\left(E_{\mathrm{F}}\right)$.

Pour les ions de terre rare, les fréquences de relaxation estimées à l'aide de la relation (19), valent $\left(\operatorname{avec}\left|J_{\text {ex }} n\left(E_{\mathrm{F}}\right)\right| \simeq 0,02\right)$ :

$$
W^{\text {E.C. }} \sim 10^{8} T \mathrm{~Hz}(T \text { en } \mathrm{K}) .
$$

2.3 Relaxation SPIN-SPIN. - Dans un composé contenant une forte concentration d'ions magnétiques, l'interaction d'échange joue un rôle prépondérant à basse température (lorsque l'échange est faible, et lorsque les distances interioniques ne sont pas trop grandes, l'interaction dipolaire magnétique est à considérer).

Dans les composés intermétalliques de terre rare par exemple, l'interaction d'échange entre deux sites est transférée par l'intermédiaire de la bande de conduction (interaction RKKY) :

$$
\mathcal{H}_{\mathrm{ech}}=\frac{1}{2} \sum_{i, j} J\left(R_{i j}\right) \mathbf{J}_{i} \cdot \mathbf{J}_{j},
$$

où $J\left(R_{i j}\right)$ est la constante d'échange, fonction de la distance $R_{i j}$ entre les deux sites $\underline{i}$ et $\underline{j}$, qui portent les moments $\mathbf{J}_{i}$ et $\mathbf{J}_{j}$.

Nous allons considérer en fait une impureté, couplée par une constante d'échange $J^{\prime}$ aux $z_{0}$ ions plus proches voisins de la matrice, dans la zone paramagnétique.

Le moment $\mathbf{G}$ de cette impureté relaxe grâce à l'interaction d'échange :

$$
\mathscr{H}_{1}=J^{\prime} \mathbf{G} \sum_{i=1}^{z_{0}} \mathbf{J}_{i} .
$$

Le bain est alors le "réservoir d'échange ", et sa densité spectrale possède une largeur : $\Delta \omega \sim \overline{J\left(R_{i j}\right)} / \hbar$, où $\overline{J\left(R_{i j}\right)}$ représente une moyenne sur les sites [1]. Pour fixer un ordre de grandeur, la constante $J$ est de l'ordre de $10 \mathrm{~K}$ dans les composés de terre rare (sauf pour l'ion $\mathrm{Gd}^{3+}$ ); cela implique :

$$
\Delta \omega \sim J / \hbar \sim 10^{12} \mathrm{~Hz} .
$$

On peut estimer, à l'aide de la formule (3), la fréquence de relaxation par échange, lorsque l'énergie de la transition $\hbar \omega_{a b}$ de l'impureté est faible devant $\Delta \omega$ (approximation du spectre blanc, cf. (7) et (8)) :

$$
W^{\text {ech }} \sim \frac{1}{\hbar} J^{\prime 2} / \Delta \omega \simeq J^{\prime 2} / \bar{J}
$$

La condition (17) pour pouvoir traiter l'interaction d'échange comme une perturbation s'écrit alors :

$$
W \ll \frac{1}{\tau_{\mathrm{c}}}=\Delta \omega \Rightarrow J^{\prime 2} / \bar{J} \ll \bar{J},
$$

ou bien :

$$
J^{\prime} \ll \bar{J}
$$

Pour une impureté couplée par échange aux ions de la matrice moins fortement que ces ions ne sont couplés entre eux, le traitement en perturbation est applicable.

Par contre, si l'on considère le cas où "l'impureté » est identique aux ions de la matrice $\left(\bar{J}=J^{\prime}\right)$, on a : $W^{\text {éch }} \sim \bar{J} \sim 1 / \tau_{c}$. Un ion interagissant ainsi par échange avec ses voisins ne peut plus être considéré comme étant faiblement couplé au « réservoir d'échange ", et le calcul de la forme du signal observé ne peut pas être effectué dans une hypothèse de perturbation.

Ces problèmes seront abordés plus en détail dans le $\S 6$.

\section{Analyse du signal en temps et en énergie.}

Les techniques mesurant la réponse du système à une perturbation statique à l'équilibre thermique, telles que la susceptibilité magnétique $\chi_{0}$, ou bien utilisant un phénomène de diffusion élastique (spectres de diffraction des neutrons par les moments magnétiques ordonnés) ne sont pas sensibles aux effets de relaxation.

Par contre, dans les techniques dites « spectroscopiques ", qui consistent à étudier l'absorption ou l'émission d'énergie par le système électronique ou nucléaire à l'aide d'une « sonde " (rayonnement électromagnétique ou neutrons), l'analyse du spectre permet d'obtenir des informations sur les fluctuations électroniques.

La sonde est couplée à une variable quantique $\tilde{M}$ $\mathrm{du}$ système atomique par une interaction que l'on peut représenter ainsi :

$$
\mathscr{H}_{\mathrm{int}}=-\tilde{M} \cdot \tilde{H} .
$$

La grandeur $\tilde{H}$ représente le champ électromagnétique associé aux photons ou le moment magnétique du neutron. En ce qui concerne les mesures directes sur le système électronique, l'opérateur $\tilde{M}$ couplé à la sonde est une composante du moment magnétique électronique (R.P.E., susceptibilité alternative, diffusion des neutrons) ou du moment dipolaire électrique (spectroscopie optique).

Dans le cas des mesures " hyperfines ", l'opérateur $\tilde{M}$ est une variable nucléaire (effet Mössbauer, C.A.P., R.M.N.). La dynamique du moment magnétique électronique est alors perçue par l'intermédiaire de l'interaction hyperfine.

La complémentarité quantique des variables temps et énergie se manifeste dans l'observation des phénomènes de relaxation. Ceux-ci peuvent en effet être appréhendés soit en tant que fluctuations temporelles, soit en tant qu'échanges énergétiques. On peut donc classifier les méthodes de mesure de la relaxation suivant la nature de l'analyse effectuée : en fonction du temps ou de l'énergie. Les deux types d'expériences 
donnent bien sûr accès aux mêmes constantes microscopiques de couplage ion-réseau. Lorsque, à l'aide d'une même technique, il est possible d'effectuer les deux types d'analyse (R.P.E. et R.M.N.), les dépendances temporelles et énergétiques sont reliées par la transformation de Fourier.

3.1 ANALYSE EN TEMPS. - Ces expériences sont réalisées en deux étapes : on commence par amener le système électronique hors équilibre thermique à l'aide d'une perturbation adéquate. En termes de matrice densité $\sigma$ du système, cela consiste à créer des éléments de matrice hors diagonaux non nuls de $\sigma$, ou bien à écarter les éléments diagonaux de $\sigma$ de leur valeur à l'équilibre de Boltzmann.

Dans la seconde étape, on mesure, après coupure de la perturbation, l'évolution temporelle d'une observable $Q$ du système atomique (par exemple l'aimantation) vers sa valeur à l'équilibre. A chaque instant, la valeur moyenne $\langle Q\rangle$ de l'observable est donnée par :

$$
\langle Q\rangle(t)=\operatorname{Tr}[Q \sigma(t)]=\sum_{m, n}\langle m|Q| n\rangle \sigma_{n m}(t),
$$

où $|m\rangle$ et $|n\rangle$ sont les états propres de l'hamiltonien $\mathscr{H}_{0}$ du système atomique. En dérivant, on obtient :

$$
\frac{\mathrm{d}\langle Q\rangle}{\mathrm{d} t}=\sum_{m, n}\langle m|Q| n\rangle \frac{\mathrm{d} \sigma_{n m}(t)}{\mathrm{d} t} .
$$

L'évolution de la matrice densité est contenue dans les expressions (13) et (14). Celle de $\langle Q\rangle$ s'en déduit donc simplement, en fonction de la matrice $S$ de relaxation.

Par exemple, pour un spin $S=1 / 2$, les composantes de l'aimantation moyenne sont reliées aux 4 éléments de la matrice densité :

$$
\left.\begin{array}{l}
\left\langle S_{\mathrm{z}}\right\rangle=\frac{1}{2}\left(\sigma_{++}-\sigma_{--}\right) \\
\left\langle S_{+}\right\rangle=\sigma_{-+} \\
\left\langle S_{-}\right\rangle=\sigma_{+-}
\end{array}\right\}
$$

En présence de relaxation électronique, décrite par l'interaction fluctuante :

$$
\mathscr{H}_{1}=-g \mathbf{S} \cdot \mathbf{H}_{1}(t),
$$

le calcul semi-classique de l'évolution temporelle de $\sigma$, effectué dans la référence [12], conduit, par exemple pour $\left\langle S_{z}\right\rangle$, à l'équation :

$$
\frac{\mathrm{d}}{\mathrm{d} t}\left\langle S_{\mathrm{z}}\right\rangle=-\frac{1}{T_{1}}\left\langle S_{\mathrm{z}}\right\rangle
$$

où :

$$
\begin{aligned}
\frac{1}{T_{1}}= & -S_{++,+}=\frac{1}{2 \hbar^{2}} \times \\
& \times \int_{-\infty}^{+\infty} \mathrm{d} \tau \mathrm{e}^{i \omega_{0} \tau} g^{2}\left\langle H_{1}^{-}(0) H_{1}^{+}(-\tau)\right\rangle,
\end{aligned}
$$

$\hbar \omega_{0}$ étant la séparation en énergie (Zeeman) entre les deux états de spin $S=1 / 2$.

L'équation (29), dont la solution est :

$$
\left\langle S_{\mathrm{z}}\right\rangle=\left\langle S_{\mathrm{z}}\right\rangle_{0} \mathrm{e}^{-t / T_{1}},
$$

implique que $\left\langle S_{z}\right\rangle$ relaxe vers la valeur 0 aux temps longs, alors qu'en fait la valeur $\left\langle S_{\mathbf{z}}\right\rangle_{\mathrm{B}}$ à l'équilibre thermique n'est en général pas nulle. Ceci vient du fait, qui a déjà été souligné, que le calcul semi-classique équivaut à l'approximation haute température. Un traitement quantique du problème [1] conduit dans la plupart des cas à l'équation d'évolution suivante $\left({ }^{4}\right)$ :

$$
\frac{\mathrm{d}\left\langle S_{\mathrm{z}}\right\rangle}{\mathrm{d} t}=-\frac{1}{T_{1}}\left[\left\langle S_{\mathrm{z}}\right\rangle-\left\langle S_{\mathrm{z}}\right\rangle_{\mathrm{B}}\right] .
$$

L'observation de l'évolution temporelle d'une quantité proportionnelle à $\left\langle S_{\mathrm{z}}\right\rangle$ (comme la puissance de radiofréquence absorbée en R.P.E.) après la perturbation permet de mesurer le «temps de relaxation $" T_{1}$ :

$$
\left\langle S_{z}\right\rangle=\left\langle S_{z}\right\rangle_{B}+\left(\left\langle S_{z}\right\rangle_{0}-\left\langle S_{z}\right\rangle_{B}\right) e^{-t / T_{1}} .
$$

La formule (30) montre que $1 / T_{1}$ est proportionnel à la composante de Fourier à l'énergie $\hbar \omega_{0}$ de la fonction de corrélation du champ fluctuant transverse $\mathrm{H}_{1}^{+}$; en effet, seuls les termes $\mathrm{H}_{1}^{+} \mathrm{S}_{-}$et $\mathrm{H}_{1}^{-} \mathrm{S}_{+}$contribuent à faire varier les populations des deux niveaux, dont la différence est proportionnelle à $\left\langle S_{z}\right\rangle$ (cf. (27)). $T_{1}$ est appelé " temps de relaxation longitudinal ».

Les expériences d'analyse temporelle du signal sont bien entendu limitées par le pouvoir de résolution en temps $\delta t$ de l'appareillage; il est nécessaire que : $\delta t \ll T_{1}$. Actuellement, ce pouvoir de résolution permet de mesurer des temps plus longs que $10^{-7} \mathrm{~s}$ dans le domaine radio-fréquence et optique, et $10^{-8} \mathrm{~s}$ dans les corrélations angulaires $\gamma-\gamma$.

3.2 ANALYSE EN ÉNERGIE. - L'analyse en énergie consiste à mesurer la réponse du système électronique, en équilibre thermique, à la perturbation introduite par la « sonde" (photon ou neutron). On s'arrange donc pour que les transitions induites par l'interaction (24) avec la sonde ne perturbent pas sensiblement l'équilibre de Boltzmann au sein du système électronique.

Nous allons examiner en premier lieu les techniques utilisant la radiation électromagnétique : on se place soit hors résonance (susceptibilité alternative), soit à la résonance (R.P.E., R.M.N., spectroscopie Mössbauer). Dans ce dernier cas, l'énergie du photon

( $\left.{ }^{4}\right)$ Les équations (29) et (31) sont exactes pour un spin $S=1 / 2$. Dans le cas général, elles sont vraies lorsque les écarts aux populations de Boltzmann $p_{i}^{\mathbf{B}}$ sont faibles, c'est-àdire lorsque $: \frac{p_{i}-p_{i}^{\mathrm{B}}}{p_{i}^{\mathrm{B}}}$ est petit, où $p_{i}$ est la population de l'état $i$. 
incident est proche d'une fréquence propre $\omega_{a b} d u$ système, et on observe des "raies " correspondant aux transitions spectroscopiques d'absorption ou d'émission d'énergie.

En spectroscopie résonnante l'analyse du spectre est effectuée au moyen d'un balayage autour de l'énergie $\hbar \omega_{a b}$ d'une transition. Ce balayage est en général périodique (de période $T$ ), et doit être lent par rapport à "l'équivalent en temps" $\hbar / \Gamma$ de la largeur $\Gamma$ de la raie spectroscopique pour obtenir une bonne résolution en énergie $\delta E$. En effet, le principe d'incertitude temps-énergie relie $T$ et $\delta E$ : $T . \delta E \simeq \hbar . \mathrm{Si}: T \gg \hbar / \Gamma$, alors $: \delta E \simeq \hbar / T \ll \Gamma$. La condition de balayage lent assure donc une résolution en énergie bien inférieure à la largeur de raie : $\delta E \ll \Gamma$.

L'interaction (24), avec un photon d'énergie $\hbar \omega$ $(\omega>0)$, se met sous la forme :

$$
\mathcal{H}_{\mathrm{int}}=-\alpha \tilde{M} \cdot \tilde{h} \mathrm{e}^{i \omega t}+\text { C.C. }
$$

Nous supposons que $\tilde{M}$ est un opérateur sans dimension du système atomique, $\alpha$ représentant le couplage avec la sonde. Lorsque $\tilde{M}$ est une observable du système (par exemple le moment magnétique), $\tilde{M}$ est hermitique $: \tilde{M}=\tilde{M}^{+}$.

La probabilité de transition entre niveaux s'obtient alors à partir de la règle d'or de Fermi (dans la suite de ce paragraphe, nous prendrons : $\hbar=1$ ) :

$P_{a \lambda, b \mu}^{\prime}(\omega)=2 \pi \alpha^{2} h^{2}|\langle b, \mu|\tilde{M}| a, \lambda\rangle|^{2} \delta\left(\omega-\omega_{a \lambda, b \mu}\right)$.

Les états $|a\rangle$ et $|b\rangle$ sont les états quantiques du système (ion et/ou noyau), $|\lambda\rangle$ et $|\mu\rangle$ représentent les variables associées au "bain " interagissant avec l'ion, et : $\omega_{a \lambda, b \mu}=\omega_{b \mu}-\omega_{a \lambda}$, où $\omega_{b \mu}$ et $\omega_{a \lambda}$ sont les énergies propres du hamiltonien du solide :

$$
\mathscr{H}=\mathscr{H}_{0}+\mathscr{H}_{b}+\mathscr{H}_{1}, \mathscr{H}|a, \lambda\rangle=\omega_{a \lambda}|a, \lambda\rangle,
$$

défini au $\S 2$. Dans la formule (34), nous supposons : $\omega_{a \lambda, b \mu}>0$.

Convenons de désigner par $\tilde{M}$ (respectivement $\tilde{M}^{+}$) l'opérateur induisant des transitions spectroscopiques d'absorption (resp. émission). L'expression (34) de $P_{a \lambda, b u}^{\prime}(\omega)$ décrit alors la probabilité a priori d'émission ou d'absorption induite d'un photon d'énergie $\hbar \omega$. $\mathrm{Au}$ sein du solide à température $T$, la probabilité d'absorption entre les états $|a, \lambda\rangle$ et $|b, \mu\rangle$, tels que : $\omega_{a \lambda, b \mu}>0$, est :

$$
P^{a}(\omega)=2 \pi \alpha^{2} h^{2} p_{a \lambda}|\langle b, \mu|\tilde{M}| a, \lambda\rangle|^{2} \delta\left(\omega-\omega_{a \lambda, b \mu}\right) .
$$

De même, la probabilité d'émission peut s'écrire

$P^{\mathrm{e}}(\omega)=2 \pi \alpha^{2} h^{2} p_{b \mu}\left|\left\langle a, \lambda\left|\tilde{M}^{+}\right| b, \mu\right\rangle\right|^{2} \delta\left(\omega-\omega_{a \lambda, b \mu}\right)$, où

$$
p_{a \lambda}=\frac{\mathrm{e}^{-\omega_{a \lambda} / k_{B} T}}{Z} .
$$

La condition suivante, analogue au principe de l'équilibre microscopique, est réalisée :

$$
\frac{P^{\mathrm{a}}(\omega)}{P^{\mathrm{e}}(\omega)}=\mathrm{e}^{\omega_{a \lambda, b_{\mu} / k_{B} T}}
$$

L'interaction avec le champ électromagnétique confère en fait aux transitions une largeur "intrinsèque » $\Gamma_{\text {in }}$; plus précisément, la distribution en énergie des photons émis ou absorbés est une lorentzienne de largeur $\Gamma_{\text {in }}$ [13]. Cette largeur varie proportionnellement à $\omega^{2 l+1}$, où $\omega$ est l'énergie de la transition induite, et $l$ le moment angulaire du photon $(l=1$ pour la R.P.E. et la R.M.N., ainsi que pour la spectroscopie optique). La probabilité d'absorption $P^{\mathrm{a}}(\omega)$, par exemple, s'écrit alors :

$$
P^{\mathrm{a}}(\omega)=p_{a \lambda}|\langle b, \mu|\tilde{M}| a, \lambda\rangle|^{2} \frac{\Gamma_{\mathrm{in}} / 2}{\Gamma_{\mathrm{in}}^{2} / 4+\left(\omega-\omega_{a \lambda, b \mu}\right)^{2}}
$$

où nous avons omis la constante de couplage $\alpha$ et l'intensité $h$ du champ de photons $\left({ }^{5}\right)$.

On peut montrer [1] que, pour des énergies $\omega$ faibles, comme celles utilisées en R.P.E. $(\omega \simeq 10 \mathrm{GHz})$ ou en R.M.N. $(\omega \simeq 10 \mathrm{MHz})$, la largeur intrinsèque est négligeable, et qu'on peut la considérer comme nulle. Par contre, pour des énergies de l'ordre des transitions nucléaires $\left(10 \mathrm{keV} \sim 10^{18} \mathrm{~Hz}\right), \Gamma_{\text {in }}$ devient plus importante et vaut entre $1 \mathrm{MHz}$ et $1 \mathrm{GHz}$ pour les isotopes Mössbauer usuels. Comme $\Gamma_{\text {in }}$ est de l'ordre des énergies hyperfines (ou inférieure), elle ne peut pas être considérée comme négligeable en spectroscopie Mössbauer. Pour des photons optiques $\left(\omega \simeq 10^{14} \mathrm{~Hz}\right)$ les largeurs radiatives sont très variables, et sont habituellement bien inférieures aux « élargissements statiques » des raies (voir ci-dessous).

Dans le cas de la diffusion inélastique des neutrons, on mesure la section efficace différentielle de perte ou de gain d'énergie d'un faisceau monochromatique de neutrons, dont l'énergie $\omega_{0}$ est choisie supérieure

(5) Cette formule, ainsi d'ailleurs que l'interaction (24), n'est valable en fait que pour $l=1$ (interaction vectorielle avec la sonde). En spectroscopie Mössbauer, où les transitions peuvent mettre en jeu des photons de moment angulaire supérieur à 1 , il convient de remplacer : $|\langle b \mu|\tilde{M}| a \lambda\rangle|^{2}$ par :

$$
\sum_{M=-l}^{l}\left|\left\langle b \mu\left|T_{l M}\right| a \lambda\right\rangle\right|^{2},
$$

où l'opérateur tensoriel $T_{l M}$ est le moment $2^{l}$-polaire « dynamique " du noyau n'ayant d'élément de matrice qu'entre l'état fondamental du noyau et son état excité. 
à l'énergie des transitions $\omega_{a b}$ que l'on souhaite observer. Le moment magnétique du neutron interagit avec les électrons magnétiques de l'ion, et dans le cas d'un moment localisé, la section efficace de perte (resp. de gain) d'énergie du neutron est décrite par l'expression (37) de $P^{\mathrm{a}}(\omega)$ (resp. $P^{\mathrm{e}}(\omega)$ ), où $\tilde{M}$ est une composante du moment magnétique de l'ion. Cependant, la largeur intrinsèque $\Gamma_{\text {in }}$ dans cette expression est à remplacer par un élargissement d'une autre nature, dont l'origine est discutée ci-dessous.

Expérimentalement, le problème de la largeur de la raie spectroscopique est un problème important. L'effet de la relaxation, comme nous le verrons, se traduit par un élargissement " dynamique » de la raie. Il est donc essentiel de connaître les autres types d'élargissement présents, afin d'évaluer correctement la contribution dynamique. En plus de l'élargissement intrinsèque $\Gamma_{\text {in }}$ dans le cas des techniques électromagnétiques, on peut distinguer deux contributions " parasites" à la largeur de raie :

i) il existe en général dans les solides une distribution des énergies des transitions (de champ cristallin, Zeeman ou hyperfines) due aux inhomogénéités cristallines, aux interactions résiduelles statiques entre les ions, ou à l'inhomogénéité du champ appliqué.

On ne connaît pas en général la forme de cette distribution. Pour simplifier, nous la supposerons dans la suite de forme lorentzienne, de largeur $\Gamma_{\text {ex }}$, conférant aux transitions un élargissement " extrinsèque " $\Gamma_{\mathrm{ex}}$. Dans les alliages dilués, cette approximation décrit assez bien la réalité.

ii) La seconde source d'élargissement vient de la "fonction d'appareil ", et rend compte de l'imprécision d'origine instrumentale : $\Gamma_{\text {instr }}$. Ce type d'élargissement donne une contribution importante par exemple dans les spectres de diffusion des neutrons par les ions paramagnétiques : l'élargissement $\Gamma_{\text {instr }}$ dépend de l'énergie incidente des neutrons et des caractéristiques du spectromètre utilisé.

En première approximation, nous supposerons que la distribution des inhomogénéités des énergies de transitions et la fonction d'appareil sont toutes deux de forme lorentzienne.

Il est commode de définir dans ces conditions une largeur expérimentale totale de la raie spectroscopique, nommée également " élargissement statique » :

$$
\Gamma_{\text {exp }}=\Gamma_{\text {ex }}+\Gamma_{\text {in }}+\Gamma_{\text {instr }} .
$$

A titre d'exemple, la décomposition de la largeur statique d'une raie du spectre Mössbauer d'une impureté de ${ }^{170} \mathrm{Yb}^{3+}$ dans une matrice d'or est la suivante :

avec :

$$
\Gamma_{\exp }=3,4 \mathrm{~mm} / \mathrm{s},
$$

$\Gamma_{\text {in }}=1 \mathrm{~mm} / \mathrm{s} ; \quad \Gamma_{\text {ex }}=0,7 \mathrm{~mm} / \mathrm{s}, \quad \Gamma_{\text {instr }}=1,7 \mathrm{~mm} / \mathrm{s}$

$\left(1 \mathrm{~mm} / \mathrm{s}\right.$ est équivalent à $68 \mathrm{MHz}$ pour $\left.{ }^{170} \mathrm{Yb}\right)$.
Nous prendrons alors pour la probabilité $P^{\mathbf{a}}(\omega)$ l'expression (37), où $\Gamma_{\text {in }}$ est remplacé par $\Gamma_{\text {exp }}$. Cette formule que l'on peut qualifier de semi-empirique, représente souvent la seule possibilité offerte pour interpréter les élargissements de raie tirés de l'expérience.

Si l'on suppose au départ le système quantique découplé du bain, ce qui correspond à une situation "statique" (pas de transitions entre niveaux du système autres que celles induites par la sonde), la probabilité d'absorption du quantum $\omega$ lors de l'une des transitions spectroscopiques s'écrit :

$$
P^{\mathrm{a}}(\omega)=\sum_{a, b}^{\prime} p_{a}|\langle b|\tilde{M}| a\rangle|^{2} \frac{\Gamma_{\exp } / 2}{\Gamma_{\text {exp }}^{2} / 4+\left(\omega-\omega_{a b}\right)^{2}},
$$

où le signe $\sum^{\prime}$ signifie que la somme sur $|a\rangle$ et $|b\rangle$ est limitée aux états tels que : $\omega_{a b}>0$. De même la probabilité que le système atomique cède l'énergie $\omega$ vaut :

$$
P^{\mathrm{e}}(\omega)=\sum_{a, b}^{\prime} p_{b}\left|\left\langle a\left|\tilde{M}^{+}\right| b\right\rangle\right|^{2} \frac{\Gamma_{\exp } / 2}{\Gamma_{\exp }^{2} / 4+\left(\omega-\omega_{a b}\right)^{2}} .
$$

On peut définir le «spectre des probabilités de transition $»$ :

$$
\begin{array}{r}
P(\omega)=P^{\mathrm{a}}(\omega)+P^{\mathrm{e}}(\omega)=\sum_{a, b} p_{a}|\langle b|\tilde{M}| a\rangle|^{2} \times \\
\times \frac{\Gamma_{\text {exp }} / 2}{\Gamma_{\text {exp }}^{2} / 4+\left(\omega-\omega_{a b}\right)^{2}},
\end{array}
$$

$$
\begin{aligned}
P(\omega)=\sum_{a, b}^{\prime}\left(p_{a}+p_{b}\right)|\langle b|\tilde{M}| a\rangle|^{2} \times \\
\times \frac{\Gamma_{\text {exp }} / 2}{\Gamma_{\text {exp }}^{2} / 4+\left(\omega-\omega_{a b}\right)^{2}} .
\end{aligned}
$$

Cette quantité, comme nous le verrons, est reliée simplement aux signaux mesurés; en outre, $\frac{1}{\pi} P(\omega)$ est normé. En effet :

$$
\begin{aligned}
& \frac{1}{\pi} \int_{0}^{\infty} P(\omega) \mathrm{d} \omega=\sum_{a, b} p_{a}|\langle b|\tilde{M}| a\rangle|^{2}= \\
& =\sum_{a} p_{a}\left\langle a\left|\tilde{M}^{+} \tilde{M}\right| a\right\rangle=\operatorname{Tr}\left(\sigma_{\mathrm{B}} \tilde{M}^{+} \tilde{M}\right),
\end{aligned}
$$

où $\sigma_{\mathrm{B}}$ est la matrice densité à l'équilibre de Boltzmann. Comme : $\tilde{M}^{+} \tilde{M}=I$, on a :

$$
\frac{1}{\pi} \int_{0}^{\infty} P(\omega) \mathrm{d} \omega=\operatorname{Tr}\left(\sigma_{\mathrm{B}}\right)=1
$$

Lorsque les phénomènes de relaxation sont négligeables, le "spectre des probabilités de transition" est donc 
formé de raies lorentziennes, centrées en $\omega=\omega_{\mathrm{b}}-\omega_{\mathrm{a}}$, de largeur à mi-hauteur $\Gamma_{\text {exp }}$, et de poids :

$$
\begin{aligned}
F_{a b}=\left(p_{a}+p_{b}\right) & |\langle b|\tilde{M}| a\rangle|^{2}= \\
& =p_{a}\left(1+\mathrm{e}^{-\omega_{a b} / k_{\mathrm{B}} T}\right)|\langle b|\tilde{M}| a\rangle|^{2} .
\end{aligned}
$$

Si maintenant on introduit le couplage de relaxation $\mathscr{H}_{1}$ entre le système atomique et le bain, il est nécessaire, dans l'évaluation de $P(\omega)$, de procéder à une moyenne sur les états du bain, dont les variables ne sont pas mesurées par la sonde spectroscopique. Pour cela, il est utile de transformer quelque peu l'expression du spectre de probabilités $P(\omega)$ [14]. Le formalisme est alors semblable à celui développé au $\S 2$ lors de la description perturbative de la relaxation.

On peut écrire, par exemple, pour une transition d'absorption :

$$
\begin{aligned}
P_{a \lambda, b \mu}^{\mathrm{a}}(\omega) & =p_{a \lambda}|\langle b, \mu|\tilde{M}| a, \lambda\rangle|^{2} \times \\
\times & \operatorname{Re}\left\{\int_{0}^{\infty} \mathrm{d} t \mathrm{e}^{i\left(\omega-\omega_{a \lambda, b \mu}\right) t-\Gamma t / 2}\right\}\left(\Gamma=\Gamma_{\text {exp }}\right) .
\end{aligned}
$$

En introduisant l'opérateur $\tilde{M}(t)$ en représentation de Heisenberg :

$$
\tilde{M}(t)=\mathrm{e}^{i J e t} \tilde{M} \mathrm{e}^{-i J e t},
$$

la probabilité d'absorption entre $|a \lambda\rangle$ et $|b \mu\rangle$ s'écrit :

$$
\begin{aligned}
P_{a \lambda, b \mu}^{\mathrm{a}}(\omega)= & \operatorname{Re}\left\{\int_{0}^{\infty} \mathrm{d} t \mathrm{e}^{i \omega t-\Gamma t / 2} p_{a \lambda} \times\right. \\
& \left.\times\left\langle a \lambda\left|\tilde{M}^{+}(t)\right| b \mu\right\rangle\langle b \mu|\tilde{M}| a, \lambda\rangle\right\} .
\end{aligned}
$$

La probabilité totale d'absorption par le système électronique (ou nucléaire) de l'énergie $\omega$ devient :

$$
\begin{array}{r}
P^{\mathrm{a}}(\omega)=\operatorname{Re} \int_{0}^{\infty} \mathrm{d} t \mathrm{e}^{i \omega t-\Gamma t / 2} \operatorname{Tr}\left[\sigma_{\mathrm{B}} \tilde{M}^{+}(t) \cdot \tilde{M}\right]= \\
=\operatorname{Re} \int_{0}^{\infty} \mathrm{d} t \mathrm{e}^{i \omega t-\Gamma t / 2}\left\langle\tilde{M}^{+}(t) \tilde{M}\right\rangle,
\end{array}
$$

où le signe $\langle>$ désigne une moyenne sur les variables du bain en même temps qu'une somme sur les états initiaux pondérés par leur facteur de Boltzmann.

On obtient de même la probabilité d'émission :

et

$$
P^{\mathrm{e}}(\omega)=\operatorname{Re} \int_{0}^{\infty} \mathrm{d} t \mathrm{e}^{i \omega t-\Gamma t / 2}\left\langle\tilde{M} \cdot \tilde{M}^{+}(t)\right\rangle
$$

avec

$$
P(\omega)=P^{\mathrm{a}}(\omega)+P^{\mathrm{e}}(\omega)=\operatorname{Re}[j(\omega)]
$$

$j(\omega)=\int_{0}^{\infty} \mathrm{d} t \mathrm{e}^{i \omega t-\Gamma t / 2}\left\langle\tilde{M}^{+}(t) \cdot \tilde{M}+\tilde{M} \cdot \tilde{M}^{+}(t)\right\rangle$

Le spectre des probabilités de transition apparaît donc comme la partie réelle de la transformée de Laplace (pour $p=\Gamma / 2-i \omega) j(\omega)$ d'une "fonction de relaxation ", qui est la fonction de corrélation temporelle de l'opérateur électronique (ou nucléaire) couplé à la sonde de mesure. Remarquons que $\operatorname{Re}[j(\omega)]$ est normé (relation (42)).

Le parallélisme entre les traitements perturbatifs de la relaxation et de l'interaction avec la sonde est illustré sur la figure 1 ci-dessous (dans le cas d'une technique résonnante). Pour les densités spectrales du bain, le principe de l'équilibre microscopique impose la condition: $J^{\downarrow}\left(\omega_{a b}\right) / J^{\dagger}\left(\omega_{a b}\right)=\mathrm{e}^{\omega_{a b} / k_{B} T}$. Quant à l'interaction avec la sonde, on peut montrer [34] à partir des relations (43) et (44), que les probabilités totales d'absorption et d'émission induites d'un photon, ou de perte et de gain d'énergie d'un neutron vérifient la relation :

$$
P^{\mathrm{a}}(\omega) / P^{\mathrm{e}}(\omega)=\mathrm{e}^{\omega / k_{\mathbf{B}} T}
$$

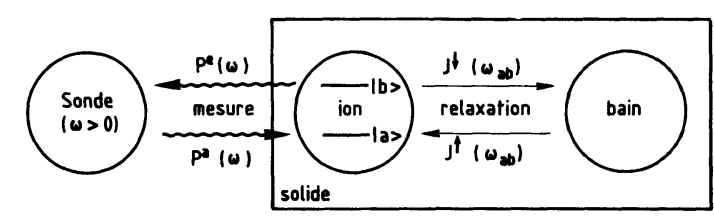

Fig. 1. - Schéma des échanges d'énergie entre l'ion et la sonde (mesure) et entre l'ion et le bain (relaxation).

[Schematic diagram of energy exchanges between the ion and the probe (measurement) and between the ion and the bath (relaxation).]

La densité spectrale complexe $j(\omega)$ joue un rôle important dans la théorie de la réponse linéaire [30]. Nous verrons que toutes les «formes de raie " mesurées par les diverses techniques considérées ici s'expriment simplement en fonction de $j(\omega)$.

La susceptibilité complexe $\chi(\omega)$, en particulier, est définie par $[30,34]$ :

$$
\begin{aligned}
\chi(\omega)= & \chi^{\prime}(\omega)-i \chi^{\prime \prime}(\omega)= \\
& =-i \int_{0}^{\infty} \mathrm{d} t \mathrm{e}^{i \omega t-\Gamma t / 2}\left\langle\left[\tilde{M}^{+}(t), \tilde{M}\right]\right\rangle .
\end{aligned}
$$

La composante $\chi^{\prime \prime}(\omega)$ reliée à l'absorption d'énergie, vaut donc :

$$
\begin{aligned}
\chi^{\prime \prime}(\omega)=-\operatorname{Im}[\chi(\omega)]=P^{\mathrm{a}}(\omega)-P^{\mathrm{e}}(\omega) & = \\
& =P^{\mathrm{a}}(\omega)\left(1-\mathrm{e}^{-\omega / k_{\mathbf{B}} T}\right) .
\end{aligned}
$$

En utilisant (46) et (47) $: \operatorname{Re}[j(\omega)]=P^{\mathrm{a}}(\omega)\left(1+\mathrm{e}^{\omega / k_{\mathbf{B}} T}\right)$, et

$$
\chi^{\prime \prime}(\omega)=\text { th } \frac{\omega}{2 k_{\mathrm{B}} T} \operatorname{Re}[j(\omega)]
$$


On reconnaît ici le théorème de fluctuation-dissipation $[15 b, 34]$ reliant l'absorption d'énergie par le système en réponse à une perturbation et la densité spectrale des fluctuations à l'équilibre thermique.

Pour effectuer le calcul de la forme de raie, ou, ce qui revient au même, de $j(\omega)$ à l'aide de l'expression (46), il faut déterminer la fonction de relaxation (par exemple d'absorption) $\left(^{6}\right)$ :

$$
G(t)=\left\langle M^{+}(t) M\right\rangle=\operatorname{Tr}_{\mathrm{at}} \overline{\left\{M^{+}(t) M \sigma_{\mathrm{B}}\right\}} .
$$

La quantité $\sigma_{\mathrm{B}}$ représente la matrice densité du système électronique (ou électronucléaire) à l'équilibre de Boltzmann $\left({ }^{7}\right)$, et le trait une moyenne sur les seules variables du bain. Dans le cadre de l'approche perturbative de la relaxation, on peut utiliser l'approximation décrite au $\S 2$, et déduire la variation temporelle de $\overline{M^{+}(t)}$ à partir de celle de la matrice densité $\sigma$ [15]. En effet, à l'aide de l'opérateur d'évolution : $U(t)=$ $\mathrm{e}^{-i \text { set }}$, on peut exprimer $\mathrm{M}^{+}(t)$ et la matrice densité $D(t)$ totale du solide :

$\frac{\mathrm{d} M^{+}(t)}{\mathrm{d} t}=i\left[\mathcal{H}, M^{+}(t)\right] \Leftrightarrow M^{+}(t)=U^{+}(t) M^{+} U(t)$ $\frac{\mathrm{d} D(t)}{\mathrm{d} t}=-i[\mathscr{H}, D(t)] \Leftrightarrow D(t)=U(t) D U^{+}(t)$

Les éléments de matrice de $\overline{M^{+}(t)}$ s'écrivent alors :

$\langle a|\overline{M(t)}| b\rangle=$

$=\sum_{c, d} \overline{\left\langle a\left|U^{+}(t)\right| c\right\rangle\langle d|U(t)| b\rangle}\left\langle c\left|M^{+}\right| d\right\rangle$

$=\sum_{c, d} \bar{U}_{a b, c d}(t)\left\langle c\left|M^{+}\right| d\right\rangle$,

où la matrice $\bar{U}(t)$ à quatre indices est une « supermatrice » d'évolution. La matrice densité $\sigma$ étant définie par :

$$
\sigma(t)=\operatorname{Tr}_{\text {bain }}(D(t))=\operatorname{Tr}_{\text {bain }}\left[U(t) \sigma \rho_{\mathrm{b}} U^{+}(t)\right],
$$

on a

$$
\begin{aligned}
\sigma_{a b}(t) & =\sum_{c, d} \overline{\langle a|U(t)| c\rangle\left\langle d\left|U^{+}(t)\right| b\right\rangle} \sigma_{c d}(0) \\
& =\sum_{c, d} \bar{V}_{a b, c d}(t) \sigma_{c d}(0) .
\end{aligned}
$$

(6) Nous écrirons désormais $M$ au lieu de $\tilde{M}$.

$\left(^{7}\right)$ Dans le cas particulier de la spectroscopie Mössbauer d'émission, où l'on observe une émission spontanée, la matrice densité figurant dans l'expression $\left\langle M \cdot M^{+}(t)\right\rangle$, analogue à (50), est en fait la matrice densité du système excité à l'instant de l'émission du photon $\gamma$ par le niveau nucléaire métastable. Ce fait peut être exploité pour mesurer les fréquences de relaxation électronique (cf. article de P. Imbert [38]).
En comparant les expressions (51) et (53), on voit que les opérateurs $\bar{U}(t)$ et $\bar{V}(t)$, régissant les évolutions temporelles de $\overline{M^{+}(t)}$ et $\sigma(t)$ respectivement sont conjugués et transposés l'un de l'autre [15]. Il en résulte que les éléments de matrice $\overline{M_{a a^{\prime}}^{+}}(t)$ obéissent à une équation d'évolution semblable à (14) :

$\frac{\overline{\mathrm{d} M_{a a^{\prime}}^{+}}(t)}{\mathrm{d} t}=-i \omega_{a a^{\prime}} M_{a a^{\prime}}^{+}(t)+\sum_{b, b^{\prime}} R_{a a^{\prime}, b b^{\prime}} \overline{M_{b b^{\prime}}^{+}}(t)$,

où la matrice $R$ est transposée de la matrice de relaxation $S$, définie dans (15) et (14) $\left({ }^{8}\right)$.

Nous allons donner une application simple du calcul du spectre de probabilités $P(\omega)$ en présence de relaxation dans le cas d'une impureté de spin $S=1 / 2$, soumise à un champ magnétique extérieur $\mathbf{H}_{0}$, et à un champ de radiofréquence alternatif $\mathbf{h} \cos \omega t$ appliqué perpendiculairement à $\mathbf{H}_{0}$ (R.P.E.). L'écart Zeeman entre les deux états vaut : $\omega_{0}=g \mu_{\mathrm{B}} H_{0}$.

L'axe de quantification étant choisi suivant $\mathbf{H}_{0}$, le champ électromagnétique (sonde) $\mathbf{h}(t)$ est couplé aux composantes transversales du moment magnétique; l'opérateur induisant les transitions est $S^{+}$ou $S^{-}$. Nous nous plaçons dans le cas " haute température » $\left(k_{\mathrm{B}} T \gg \omega_{0}\right)$, réalisé dans la majorité des expériences en R.P.E. pour des champs inférieurs à $10 \mathrm{kG}$ pour calculer : $P^{\mathrm{a}}(\omega) \simeq P^{\mathrm{e}}(\omega) \simeq P(\omega) / 2$.

Nous supposons qu'il existe une certaine distribution des énergies de la transition, due aux inhomogénéités du champ appliqué et du champ cristallin, que nous prendrons de forme lorentzienne de largeur $a$. La probabilité $P^{\mathrm{a}}(\omega)$, donnée par l'expression $(4 \overline{3})$ où l'on prend : $\Gamma=a$, s'obtient en calculant $: \frac{\frac{d}{\mathrm{~d} S^{+}(t)}}{\mathrm{d} t}$, c'est-à-dire $\frac{\mathrm{d}}{\mathrm{d} t} \overline{\left\langle+\left|S^{+}(t)\right|->\right.}$ en présence de relaxation du spin $S$, induite par un champ fluctuant $\mathbf{H}_{1}(t)$. Les équations (36) de [12] donnent l'équation d'évolution de la matrice densité d'un spin $1 / 2$; en particulier :

$$
\frac{\mathrm{d} \sigma_{+-}}{\mathrm{d} t}=\left(+i \omega_{0}-\frac{1}{T_{2}}\right) \sigma_{+-}
$$

où :

$$
\begin{aligned}
\frac{1}{T_{2}}= & -S_{+-,+-} \\
= & \frac{1}{4} \int_{-\infty}^{+\infty} \mathrm{e}^{i \omega_{0} \tau} \mathrm{d} \tau g^{2}\left\langle H_{1}^{-}(0) H_{1}^{+}(-\tau)\right\rangle+ \\
& +\frac{1}{2} \int_{-\infty}^{+\infty} \mathrm{d} \tau g^{2}\left\langle H_{1}^{\mathrm{z}}(0) H_{1}^{\mathrm{z}}(-\tau)\right\rangle .
\end{aligned}
$$

$\left({ }^{8}\right)$ Les matrices $R$ et $S$ sont réelles lorsqu'on néglige les déplacements de raie du second ordre dus à l'interaction de relaxation $\mathscr{H}_{1}$, ce qui est en général le cas. 
Donc d'après (54) :

$$
\begin{aligned}
\frac{\mathrm{d}}{\mathrm{d} t} \overline{\left\langle+\left|S^{+}(t)\right|-\right\rangle}=\left(-i \omega_{0}-\frac{1}{T_{2}}\right) \overline{\left\langle+\left|S^{+}(t)\right|-\right\rangle} \Rightarrow \overline{\left\langle+\left|S^{+}(t)\right|-\right\rangle}= \\
=\mathrm{e}^{\left(-i \omega_{0}-1 / T_{2}\right) t}\left\langle+\left|S^{+}\right|-\right\rangle .
\end{aligned}
$$

La probabilité $P(\omega)$ donnée par (45) vaut donc

$$
\begin{aligned}
& P(\omega)=\operatorname{Re} \int_{0}^{\infty} \mathrm{d} t \exp \left[\left(i \omega-\frac{a}{2}\right) t\right] \exp \left[\left(-i \omega_{0}-\frac{1}{T_{2}}\right) t\right]\left\langle+\left|S^{+}\right|-\right\rangle\left\langle-\left|S^{-}\right|+\right\rangle \\
& P(\omega)=\left|\left\langle+\left|S^{+}\right|-\right\rangle\right|^{2} \frac{a / 2+1 / T_{2}}{\left(\omega-\omega_{0}\right)^{2}+\left(\frac{a}{2}+\frac{1}{T_{2}}\right)^{2}} .
\end{aligned}
$$

La raie R.P.E. est une lorentzienne centrée sur la fréquence de résonance $\omega_{0}$, et de demi-largeur : $\frac{a}{2}+\frac{1}{T_{2}}$.

L'effet de la relaxation entre les deux niveaux d'une transition unique est donc d'élargir la raie, le supplément de demi-largeur "dynamique " étant $1 / T_{2}$, donné par l'expression (54). Dans cette expression, on distingue traditionnellement l'élargissement «adiabatique ", défini par le terme contenant la fonction de corrélation de $H_{1}^{2}$, qui représente la modulation de l'écart Zeeman due à la relaxation, et l'élargissement de "temps de vie", égal à $1 / 2 T_{1}$, lié aux fluctuations transversales du champ fluctuant. $T_{2}$ est appelé " temps de relaxation transverse".

Dans le cas de fluctuations isotropes, en symétrie cubique, et à haute température $\left(k_{\mathrm{B}} T \gg \hbar \omega_{0}\right)$, on voit que :

$$
\left\langle H_{1}^{\mathrm{z}}(0) H_{1}^{\mathrm{z}}(-\tau)\right\rangle=\frac{1}{2}\left\langle H_{1}^{-}(0) H_{1}^{+}(-\tau)\right\rangle .
$$

$T_{2}$ est alors égal à $T_{1}$, donné par la formule (30), qui représente la constante de temps liée au retour à l'équilibre de $\left\langle S_{z}\right\rangle$ mesurée par la méthode d'analyse en temps.

D'une manière générale, les paramètres mesurés à l'aide des 2 types de méthodes que nous venons d'évoquer dépendent de densités spectrales des fluctuations du bain. Ces quantités contiennent l'information sur le couplage ion-réseau, et leur calcul dans différentes hypothèses fera l'objet des $\S 5$ et 6 de cet article. Le paragraphe 4 suivant est consacré à une description plus détaillée des diverses techniques sensibles à la relaxation électronique, et à l'effet de cette dernière sur les spectres en énergie.

\section{Techniques de mesure et formes de raie.}

Les techniques spectroscopiques sensibles à la relaxation paramagnétique sont en général appliquées dans des conditions expérimentales assez diverses : présence ou absence de champ magnétique, température faible ou élevée devant l'énergie du champ cristallin (pour les terres rares)... Les niveaux atomiques concernés par la relaxation sont donc variés : niveaux Zeeman du doublet fondamental d'un ion de Kramers en R.P.E., niveaux hyperfins en effet Mössbauer à basse température, plusieurs doublets de champ cristallin en D.I.N., niveaux excités de spin-orbite (pour les terres rares) en spectroscopie optique...

Chaque technique mesure donc une forme particulière de temps ou fréquence de relaxation, et il convient d'être très prudent lorsqu'on veut comparer, par exemple, les fréquences de relaxation obtenues sur un même système à l'aide de deux ou plusieurs techniques différentes. Il est en général plus intéressant de comparer entre elles les constantes de couplage microscopiques tirées des variations thermiques des fréquences de relaxation mesurées. Pour cela, il est essentiel d'avoir à l'esprit les conditions expérimentales précises dans lesquelles chaque technique particulière est mise en œuvre.

Dans les deux premières sections de ce paragraphe, nous allons détailler quelque peu les techniques spectroscopiques énumérées dans l'introduction; dans la troisième section, nous nous intéresserons à l'effet d'effacement par la relaxation de la structure fine d'un spectre en énergie, qui affecte fortement les formes de raie en R.P.E., spectroscopie Mössbauer et de neutrons inélastiques.

4.1 LeS MESURES "DIRECTES " SUR LE SYSTÈME ÉLECTRONIQUE. - a) Les observations de la dépendance en fréquence de la susceptibilité magnétique alternative longitudinale hors résonance $\chi_{\|}(\omega)$ ont représenté les premières mesures des temps de relaxation paramagnétique dans les isolants $[16,17]$.

Cette technique consiste à polariser le système de spins par un champ magnétique $\mathbf{H}_{0}$, et à observer sa réponse $\chi_{\|}(\omega)$ lorsqu'un petit champ alternatif $h \cos \omega t$ parallèle à $H_{0}$ est appliqué sur l'échantillon. Le domaine de fréquence que l'on peut explorer par cette technique va de $1 \mathrm{~Hz}$ à $10^{8} \mathrm{~Hz}$. 
Le couplage entre la sonde et une impureté de spin $S=1 / 2$ s'écrit :

$$
\mathscr{H}_{\mathrm{int}}=-g \mu_{\mathrm{B}} S_{\mathrm{z}} h \cos \omega t,
$$

si l'on choisit l'axe $\mathrm{O} z$ de quantification suivant $\mathbf{H}_{0}$.

Ce couplage est non résonnant, en ce sens qu'il ne peut pas induire de transitions entre $|+\rangle$ et $|-\rangle$, et donc ne crée pas d'éléments de matrice hors dia- gonaux $\sigma_{+-}$ou $\sigma_{-+}$du système électronique. La susceptibilité $\chi_{\|}(\omega)$ se calcule d'après l'expression (48), où : $M=S_{\mathrm{z}}$ et $\Gamma=0$, et la partie dissipative $\chi^{\prime \prime}(\omega)$ est donnée par le théorème de fluctuation-dissipation (49). Dans les conditions expérimentales usuelles : $\omega<10^{8} \mathrm{~Hz}$ et $k_{\mathrm{B}} T \gtrsim 1 \mathrm{~K}$; l'approximation haute température est valable : th $\frac{\omega}{2 k_{\mathrm{B}} T} \simeq \frac{\omega}{2 k_{\mathrm{B}} T}$. Donc :

$$
\begin{aligned}
& \chi_{\|}^{\prime \prime}(\omega)=g^{2} \mu_{\mathrm{B}}^{2} \frac{\omega}{4 k_{\mathrm{B}} T} \operatorname{Re} \int_{0}^{\infty} \mathrm{d} t \mathrm{e}^{i \omega t}\left[\left\langle S_{\mathrm{z}}(t) S_{\mathrm{z}}+S_{\mathrm{z}} S_{\mathrm{z}}(t)\right\rangle\right] \\
& \chi_{\|}^{\prime \prime}(\omega)=g^{2} \mu_{\mathrm{B}}^{2} \frac{\omega}{2 k_{\mathrm{B}} T} \operatorname{Re} \int_{0}^{\infty} \mathrm{d} t \mathrm{e}^{i \omega t}\left[\left\langle+\left|\overline{S_{\mathrm{z}}(t)} S_{\mathrm{z}}\right|+\right\rangle+\left\langle-\left|\overline{S_{\mathrm{z}}(t)} S_{\mathrm{z}}\right|-\right\rangle\right] .
\end{aligned}
$$

La variation temporelle de $\overline{S_{\mathrm{z}}(t)}$ s'obtient à partir des équations (54), formellement identiques aux équations d'évolution de $\sigma_{++}$et $\sigma_{--}$données par les expressions (36) de [12] (puisque $\sigma_{+-}=\sigma_{-+}=0$ ) :

$$
\left\{\begin{array}{l}
\frac{\mathrm{d} \sigma_{++}}{\mathrm{d} t}=-\frac{1}{2 T_{1}}\left(\sigma_{++}-\sigma_{--}\right) \\
\frac{\mathrm{d} \sigma_{--}}{\mathrm{d} t}=-\frac{1}{2 T_{1}}\left(\sigma_{--}-\sigma_{++}\right) .
\end{array}\right.
$$

On obtient simplement :

$$
\begin{aligned}
\left\langle+\left|\overline{S_{\mathbf{z}}(t)}\right|+\right\rangle-\left\langle-\left|\overline{S_{\mathbf{z}}(t)}\right|-\right\rangle= & \\
& =2 \mathrm{e}^{-t / T_{1}}\left\langle+\left|S_{\mathbf{z}}\right|+\right\rangle
\end{aligned}
$$

et :

$$
\chi_{\|}^{\prime \prime}(\omega)=g^{2} \mu_{\mathrm{B}}^{2} \frac{\omega}{k_{\mathrm{B}} T}\left|\left\langle+\left|S_{\mathrm{z}}\right|+\right\rangle\right|^{2} \operatorname{Re}\left(-\frac{1}{i \omega-1 / T_{1}}\right)
$$$$
\chi^{\prime \prime}(\omega)=\chi_{0} \frac{\omega / T_{1}}{\omega^{2}+1 / T_{1}^{2}},
$$

où $\chi_{0}$ est la susceptibilité statique de l'impureté.

Cette expression est valable tant que l'on ne tient pas compte des interactions entre les spins. $T_{1}$ désigne le temps de relaxation "spin-réseau " de l'ion considéré comme isolé.

Ĺ'absorption d'énergie non résonnante par l'impureté isolée, décrite par $\chi^{\prime \prime}(\omega)$ et liée aux fluctuations électroniques, est donc maximale lorsque la fréquence de la sonde est accordée avec la fréquence de relaxation $1 / T_{1}$.

En présence d'interactions d'échange et dipolaires entre les spins, il faut tenir compte du fait qu'il existe des fluctuations « internes " au système électronique, induites par la relaxation "spin-spin", possédant un temps caractéristique $T_{\mathrm{ss}} . T_{\mathrm{ss}}\left(\sim 10^{-6}-10^{-9} \mathrm{~s}\right)$ est en général beaucoup plus court que $T_{1}$ à basse température.

Si l'on augmente alors la fréquence d'observation de manière que : $\omega \gg 1 / T_{1}$, les échanges d'énergie spin-réseau ne contribuent plus au signal de susceptibilité, et le système électronique peut être considéré comme "découplé " du réseau. Il est alors possible d'étudier la relaxation "spin-spin " dans le domaine de fréquences : $\omega \sim 1 / T_{\text {ss }}$.

La figure 2 [18] donne l'allure schématique des courbes de dispersion $\chi^{\prime}(\omega)$ et d'absorption $\chi^{\prime \prime}(\omega)$ en fonction de la fréquence. La figure 3 [19] montre la partie basse fréquence de la susceptibilité alternative mesuree dans un sel ferrique. Le maximum de $\chi^{\prime \prime}(\omega)$ se trouvant aux alentours de $\omega \simeq 10 \mathrm{~Hz}$, le temps de relaxation spin-phonons des ions $\mathrm{Fe}^{3+}$ dans ce composé à $T=2,8 \mathrm{~K}$ est environ : $T_{1}=0,1 \mathrm{~s}$.

Cette technique a été appliquée à la mesure des temps de relaxation spin-réseau [20], mais surtout

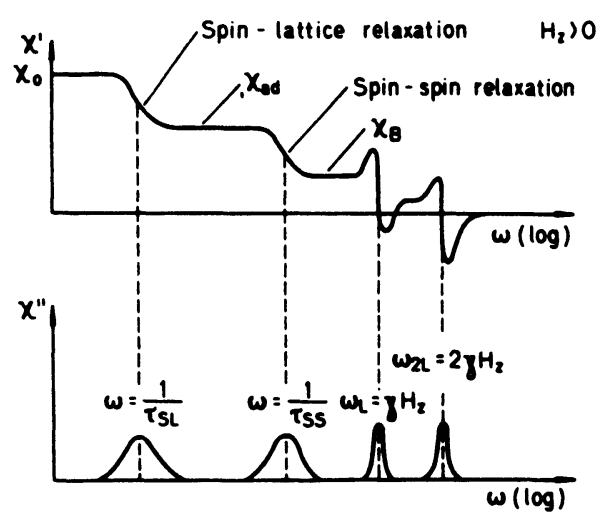

Fig. 2. - (D'après [18]) Représentation schématique des courbes de dispersion $\chi_{\|}^{\prime}(\omega)$ et d'absorption $\chi_{\|}^{\prime \prime}(\omega)$. Les parties résonnantes (en $\omega_{\mathrm{L}}$ et $\omega_{2 \mathrm{~L}}$ ) sont présentes, car l'interaction spin-spin mélange quelque peu les états propres Zeeman; le champ de radio-fréquence parallèle à $H_{0}$ peut alors induire des transitions résonnantes (de faible intensité).

[(After [18]) Schematic representation of dispersion curves $\chi_{\|}^{\prime}(\omega)$ and absorption curves $\chi_{\|}^{\prime \prime}(\omega)$. The resonant parts (at $\omega_{\mathrm{L}}$ and $\left.\omega_{2 \mathrm{~L}}\right)$ are present, because the spin-spin interaction somehow mixes the Zeeman eigen-states; the radio-frequency field parallel to $H_{0}$ may then induce resonant transitions (of weak intensity).] 


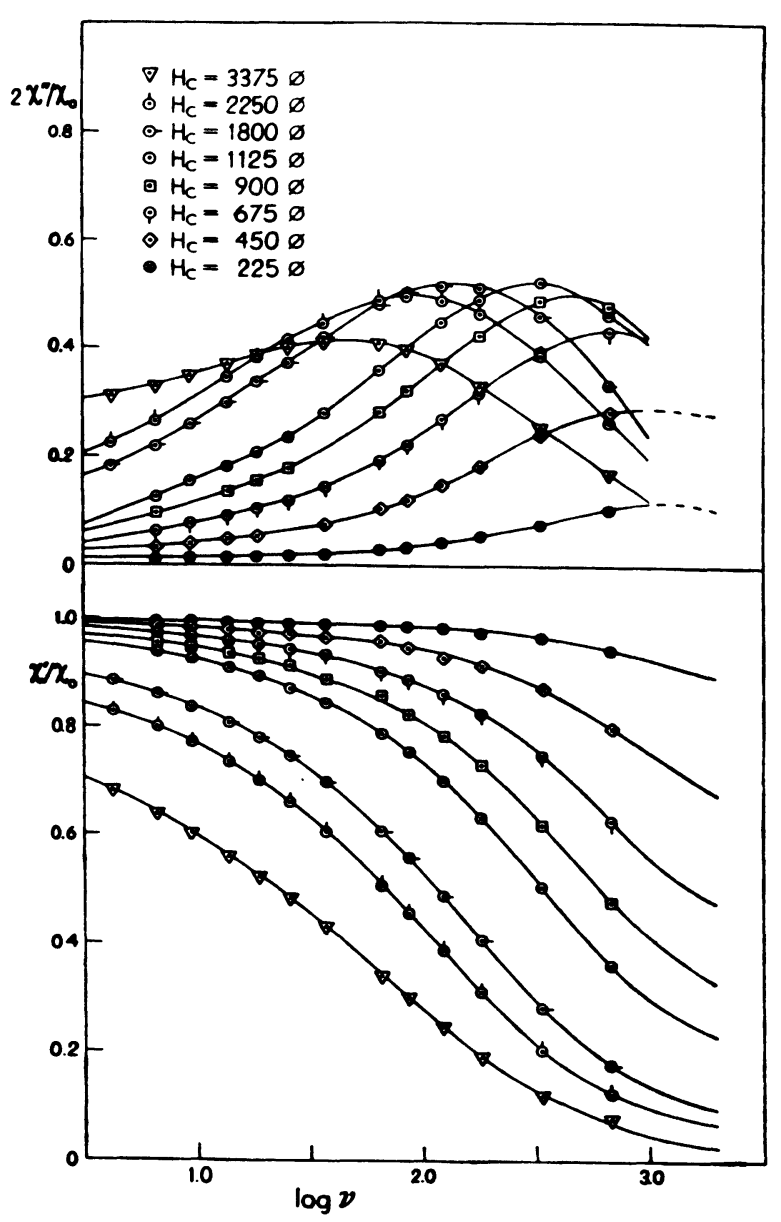

Fig. 3. - (D'après [19]) Courbes expérimentales de dispersion $\left(\chi^{\prime} \mid \chi_{0}\right)$ et d'absorption $\left(2 \chi^{\prime \prime} \mid \chi_{0}\right)$ en fonction de $\log v=$ $\log \omega / 2 \pi$ dans $\mathrm{FeNH}_{4}\left(\mathrm{SO}_{4}\right)_{2} .12 \mathrm{H}_{2} \mathrm{O}$ à $T=2,883 \mathrm{~K}$ pour différentes valeurs du champ magnétique.

[(After [19]) Experimental dispersion curves $\left(\chi^{\prime} / \chi_{0}\right)$ and absorption curves $\left(2 \chi^{\prime \prime} / \chi_{0}\right)$ as a function of : $\log v=$ $\log \omega / 2 \pi$ in $\mathrm{FeNH}_{4}\left(\mathrm{SO}_{4}\right)_{2} .12 \mathrm{H}_{2} \mathrm{O}$ at $T=2.883 \mathrm{~K}$ for different values of the magnetic field.]

à l'étude de la dépendance en champ magnétique des fréquences de relaxation spin-spin [21] dans le domaine : $\omega \sim 10^{7}-10^{8} \mathrm{~Hz}$. Cependant, à l'heure actuelle, cette méthode n'est pratiquement plus employée dans l'étude de la relaxation paramagnétique. Elle a été supplantée par les méthodes résonnantes (R.P.E., effet Mössbauer), plus sensibles et plus précises.

L'intérêt des mesures de susceptibilité alternative s'est reporté sur les "verres de spin ", dont les propriétés dynamiques dans la zone de « gel » des moments magnétiques sont sujettes à controverse. La dépendance en température de la susceptibilité alternative $\chi(\omega T)$ autour de la "température de gel » $T_{\mathrm{g}}$ a été particulièrement étudiée dans un grand nombre de ces composés (voir par exemple les Réfs. [22, 23]).

b) La grande majorité des études de relaxation électronique a toutefois été l'apanage de la résonance paramagnétique électronique. Cette technique, dont il a déjà été question, au $\S 3.2$, consiste à faire absorber de l'énergie électromagnétique par un système de moments magnétiques polarisés par un champ extérieur $\mathbf{H}_{0}$. On applique pour cela un petit champ oscillant h cos $\omega$ t perpendiculairement à $\mathbf{H}_{0}$. L'absorption résonnante se produit pour une fréquence $\omega \simeq \omega_{0}=g \mu_{\mathrm{B}} H_{0}$; pour des champs appliqués de quelques $\mathrm{kG}, \omega_{0}$ est de l'ordre de $10 \mathrm{GHz}$ (pour $g=2$ ). Dans les conditions expérimentales habituelles $(T>1 \mathrm{~K})$, l'approximation "haute température " $\left(k_{\mathrm{B}} T \gg \omega_{0}\right)$ est valable.

On a recours, en R.P.E., aux deux types de méthodes d'analyse en énergie et en temps pour mesurer les fréquences de relaxation. Nous allons les décrire successivement en nous restreignant à des cas simples, car, particulièrement dans les méthodes d'analyse temporelle, il existe une grande diversité de modes opératoires. Nous évoquerons ensuite une méthode utilisant le phénomène de saturation radiofréquence pour mesurer le temps de relaxation.

L'interaction entre la sonde et l'impureté de spin $S=1 / 2$ s'écrit dans ce cas :

$$
\mathcal{H}_{\mathrm{int}}(\omega)=-\mathrm{mh} \cos \omega t=-g \mu_{\mathrm{B}} S_{\mathrm{x}} h \cos \omega t,
$$

en choisissant l'axe $O z$ suivant $\mathbf{H}_{0}$ et l'axe $O x$, suivant $h$ (on suppose le champ de radiation incident linéairement polarisé).

La méthode d'analyse en énergie est mise en œuvre à l'aide d'un faible niveau de puissance radiofréquence. Le signal détecté est l'absorption de puissance dans l'échantillon, qui s'écrit :

$$
\begin{array}{r}
S(\omega)=2 \pi h^{2} g^{2} \mu_{\mathrm{B}}^{2} \omega\left[P^{\mathrm{a}}(\omega)-P^{\mathrm{e}}(\omega)\right]= \\
=2 \pi h^{2} g^{2} \mu_{\mathrm{B}}^{2} \omega \chi^{\prime \prime}(\omega),
\end{array}
$$

en rétablissant la constante dépendant de la sonde (expression (34)). Les probabilités $P^{\mathrm{a}}(\omega)$ et $P^{\mathrm{e}}(\omega)$ ont été définies au $\S 3.2$, ainsi que la partie dissipative de la susceptibilité $\chi^{\prime \prime}(\omega)$.

Dans la situation "haute température" de la R.P.E., $P^{\mathrm{a}}(\omega)$ et $P^{\mathbf{e}}(\omega)$ sont pratiquement égales, et l'absorption d'énergie est due à la faible différence des populations $p_{+}$et $p_{-}$des niveaux $|+\rangle$et $|-\rangle$ à l'équilibre de Boltzmann :

$$
p_{+}-p_{-}=\text {th } \frac{\omega}{2 k_{\mathrm{B}} T} \simeq \frac{\omega}{2 k_{\mathrm{B}} T} .
$$

Ceci est exprimé par la relation (47), et permet d'écrire $S(\omega)$ sous une forme plus "physique " :

$$
S(\omega)=\omega W_{\mathrm{rf}}\left(p_{+}-p_{-}\right)
$$

où :

$$
W_{\mathrm{rf}}=2 \pi h^{2} g^{2} \mu_{\mathrm{B}}^{2} \operatorname{Re}[j(\omega)] .
$$

$W_{\text {rf }}$ est la probabilité d'absorption ou d'émission induite à la fréquence $\omega$.

Lorsque les effets de la relaxation sont négligeables, 
$\operatorname{Re}[j(\omega)]$ est une lorentzienne de largeur $\Gamma_{\text {exp }}$, reflétant les inhomogénéités des énergies de transition au sein du solide, et centrée sur la fréquence de résonance $\omega_{0}$. Comme en général : $\Gamma_{\text {exp }} \ll \omega_{0}$, le signal de puissance absorbée peut s'écrire :

$$
S(\omega) \simeq 2 \pi h^{2} g^{2} \mu_{\mathrm{B}}^{2} \frac{\omega_{0}^{2}}{2 k_{\mathrm{B}} T} \operatorname{Re}[j(\omega)] .
$$

Le plus souvent le signal est observé à fréquence $\omega_{0}$ fixe, et le balayage en énergie est effectué par une modulation du champ magnétique autour de la valeur : $H_{0}=\omega_{0} / g \mu_{\mathrm{B}}$. On détecte en fait la dérivée $\mathrm{d} S(H) / \mathrm{d} H$ du signal d'absorption, ce qui permet une meilleure détermination de la position et de la largeur de la raie.

En présence de relaxation électronique, la forme de $\operatorname{Re}[j(\omega)]=P(\omega)$ a été calculée au $\S 3.2$ à partir de l'équation d'évolution de l'opérateur $\overline{S^{+}(t)}$ (expression (56)). Si $a$ est la largeur statique $\left(a=\Gamma_{\text {exp }}\right)$ de la distribution, supposée lorentzienne, des fréquences de transition, la raie est élargie d'une quantité « dynamique $" 1 / T_{2}$, et la mesure de la variation thermique de la demi-largeur à mi-hauteur :

$$
\Delta H(T)=\frac{a}{2}+\frac{1}{g \mu_{\mathrm{B}}} \frac{1}{T_{2}},
$$

permet d'obtenir celle de $1 / T_{2}$, une fois évaluée la largeur statique $a$. Le temps de relaxation transversal $T_{2}$ est défini par (55). La largeur statique $a$ peut être mesurée directement sur un spectre à très basse température, lorsque : $1 / T_{2} \ll a$. Lorsqu'on augmente la température, la fréquence de relaxation croît, et la raie s'élargit progressivement jusqu'à devenir indécelable lorsque $\Delta H \sim H_{0}$.

La figure 4 montre deux spectres obtenus, à une fréquence : $\omega_{0}=3,22 \mathrm{GHz}$, dans l'alliage dilué $\mathrm{AuYb}^{3+}$, à deux températures différentes [26]. La largeur de la raie à $T=0,16 \mathrm{~K}$ reflète principalement les élargissements statiques : $a \simeq 20 \mathrm{G} \simeq 0,6 \times 10^{8} \mathrm{~Hz}$. A $T=0,81 \mathrm{~K}$, la largeur totale est environ $50 \mathrm{G}$, ce qui correspond à un élargissement dynamique de $30 \mathrm{G}$, c'est-à-dire à : $1 / T_{2} \simeq 10^{8} \mathrm{~Hz}$ pour l'impureté $\mathrm{Yb}^{3+}$.

D'une manière générale, la "fenêtre " de mesure des fréquences de relaxation par cette méthode d'analyse est en gros :

$$
\frac{a}{10} \lesssim \frac{1}{T_{2}} \lesssim \frac{\omega_{0}}{5}, \quad \text { où } \quad \omega_{0} \sim 10 \mathrm{GHz}
$$

L'étude de la dynamique d'impuretés de terre rare dans les matrices métalliques, sous l'influence du couplage d'échange entre les électrons 4 fet les électrons de conduction, est effectuée à l'aide de cette méthode à très basse température $(T<20 \mathrm{~K})[10,27]$.

En présence de structure de la raie, c'est-à-dire de transitions spectroscopiques d'énergies légèrement différentes, les effets de la relaxation sur le spectre

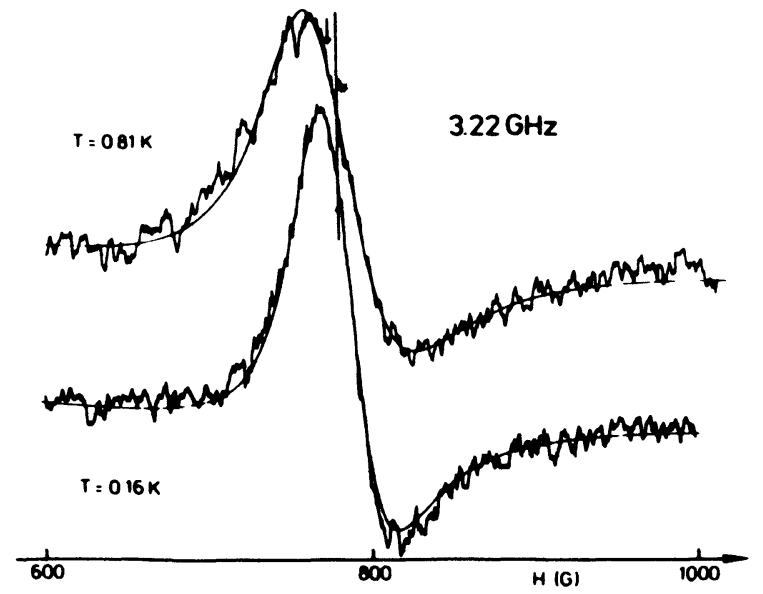

Fig. 4. - (D’après [26]) Spectres R.P.E. de $\mathrm{Yb}^{3+}$ dilué à $1200 \mathrm{ppm}$ dans Au (voir texte).

[(After [26]) E.P.R. spectra of the $\mathrm{Yb}^{3+}$ ion diluted at a level of $1200 \mathrm{ppm}$ in gold (see text).]

sont plus complexes. Ce phénomène, qui se manifeste également en spectroscopie Mössbauer et de neutrons inélastiques, sera abordé dans le $\S$ 4.3.

Les méthodes d'analyse en temps du signal sont assez diverses, et sont décrites de manière complète dans la référence [24]. Nous allons décrire brièvement la méthode de "saturation par impulsion".

Une impulsion de radiofréquence intense et brève sature la transition, c'est-à-dire égalise les populations $p_{+}$et $p_{-}$des deux niveaux. Après coupure de la perturbation, la relaxation électronique restaure l'équilibre de Boltzmann, le " rétablissement » de l'aimantation : $\left\langle S_{\mathrm{z}}\right\rangle=\frac{p_{+}-p_{-}}{2 g}$ étant régi par l'équation (32) :

$$
\frac{\mathrm{d}\left\langle S_{\mathrm{z}}\right\rangle}{\mathrm{d} t}=-\frac{1}{T_{1}}\left[\left\langle S_{\mathrm{z}}\right\rangle-\left\langle S_{\mathrm{z}}\right\rangle_{\mathrm{B}}\right] .
$$

Le retour vers la valeur d'équilibre $\left\langle S_{\mathbf{z}}\right\rangle_{\text {B }}$ s'effectue donc suivant une loi exponentielle :

$$
\left\langle S_{\mathrm{z}}\right\rangle=\left\langle S_{\mathrm{z}}\right\rangle_{\mathrm{B}}\left(1-\mathrm{e}^{t / T_{1}}\right)
$$

$\left(\operatorname{car}\left\langle S_{\mathrm{z}}\right\rangle_{0}=0\right)$.

Le temps de relaxation $T_{1}$ est alors mesuré en observant l'évolution, après l'impulsion saturante, d'une quantité proportionnelle à $\left\langle S_{z}\right\rangle$, c'est-à-dire à $p_{+}-p_{-}$. Dans la majorité des cas, on étudie l'état fondamental du système électronique, et on a souvent recours à la mesure de la puissance de faible niveau absorbée en fonction du temps (expressions (64) et 65)) à la fréquence de résonance $\omega_{0}$ :

$$
S\left(\omega_{0}, t\right)=\omega_{0} W_{\mathrm{rf}}\left(p_{+}-p_{-}\right)=2 g \omega_{0} W_{\mathrm{rf}}\left(\omega_{0}\right)\left\langle S_{\mathrm{z}}\right\rangle .
$$


A la résonance, $W_{\text {rf }}$ est maximal et vaut :

$$
W_{\mathrm{rf}}\left(\omega_{0}\right)=2 \pi h^{2} g^{2} \mu_{\mathrm{B}}^{2} \frac{1}{\frac{a}{2}+\frac{1}{T_{2}}}=2 \pi \hbar^{2} g^{2} \mu_{\mathrm{B}}^{2} T_{2}^{\prime},
$$

où :

$$
\frac{1}{T_{2}^{\prime}}=\frac{a}{2}+\frac{1}{T_{2}} .
$$

Un exemple de " rétablissement » du signal après une impulsion saturante est montré sur la figure 5 . La pente du graphe :

$$
\ln \left\{\frac{S\left(\omega_{0}, \infty\right)-S\left(\omega_{0}, t\right)}{S\left(\omega_{0}, \infty\right)}\right\}=f(t)
$$

donne le temps de relaxation $T_{1}$.

Dans certaines expériences, on observe la résonance sur un état électronique excité (de spin-orbite ou de champ cristallin), peuplé par une irradiation laser convenable. La méthode de saturation par impulsion peut alors également être utilisée pour mesurer $T_{1}$

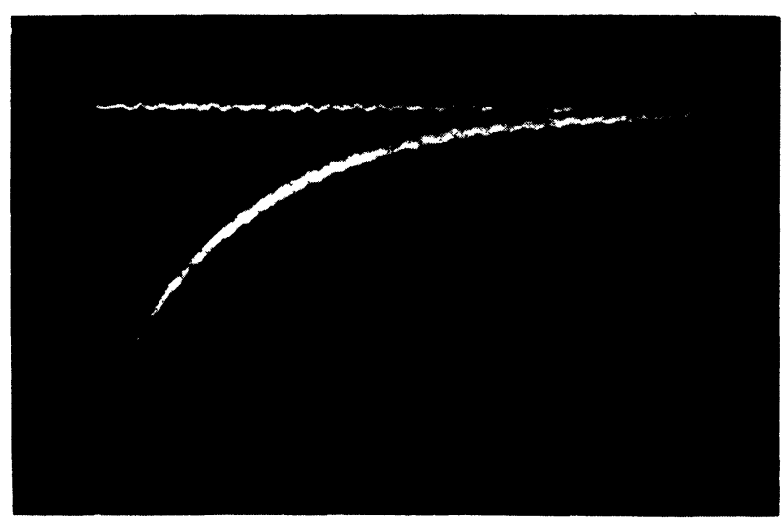

a)

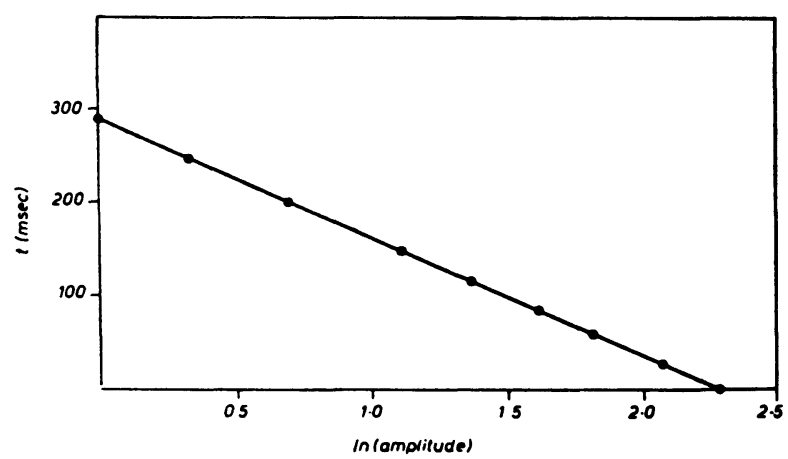

b)

Fig. 5. - (D'après [24]) La courbe expérimentale de " rétablissement " de l'aimantation, représentée en $\underline{a}$, est tracée sur une échelle semi-logarithmique en $b$.

[(After [24]) The experimental curve of magnetization « recovery ", presented in $\underline{a}$, is plotted on a semi-logarithmic scale in $b$.] dans l'état excité en suivant l'évolution temporelle de la fluorescence issue de l'un des niveaux excités [25].

Un second type de mesure en temps des fréquences de relaxation, la méthode des "échos de spins", surtout employée en R.M.N., sera décrit brièvement dans le paragraphe $4.2 c$.

A côté de ces deux méthodes "impulsionnelles", on utilise également une méthode dite de l'« onde continue " (Continuous Wave), qui n'est pas une méthode d'analyse en temps à proprement parler, mais dont l'intérêt dans cet exposé est de montrer les rôles opposés de la relaxation et de la saturation radiofréquence. La condition de non-saturation de la transition à la résonance est donnée par :

$$
W_{\text {rf }}\left(\omega_{0}\right) \ll \frac{1}{T_{1}},
$$

ou, en utilisant (70) :

$$
h^{2} g^{2} \mu_{\mathrm{B}}^{2} T_{2}^{\prime} T_{1} \ll 1 .
$$

Dans ce cas, le système électronique reste pratiquement en équilibre thermique; cette situation est celle de l'analyse en énergie. Au contraire, lorsque : $W_{\mathrm{rf}}\left(\omega_{0}\right) \gg 1 / T_{1}$, la saturation est complète, le système électronique est hors équilibre thermique pendant l'impulsion saturante.

La situation intermédiaire : $W_{\mathrm{rf}} \sim 1 / T_{1}$, peut être mise à profit pour " équilibrer " l'effet de la relaxation et créer un état stationnaire lors d'une irradiation continue de l'échantillon à l'aide d'une puissance faiblement saturante. Nous allons calculer la puissance absorbée à la résonance en fonction de $W_{\mathrm{rf}}$, en supposant qu'elle peut être exprimée par la relation (66), valable en principe en l'absence de saturation. Les populations $p_{+}$et $p_{-}$des états $|+\rangle$et $|-\rangle$ varient dans le temps sous l'influence des transitions de relaxation et de radiofréquence (cf. Fig. 6) :

$$
\left.\begin{array}{l}
\frac{\mathrm{d} p_{+}}{\mathrm{d} t}=-W_{\uparrow} p_{+}+W_{\downarrow} p_{-}-W_{\mathrm{rf}}\left(p_{+}-p_{-}\right) \\
\frac{\mathrm{d} p_{-}}{\mathrm{d} t}=-W_{\downarrow} p_{-}+W_{\uparrow} p_{+}-W_{\mathrm{rf}}\left(p_{-}-p_{+}\right) .
\end{array}\right\}
$$

Les probabilités de transition $W_{\uparrow}$ et $W_{\downarrow}$ sont dans le rapport de Boltzmann ( $\S 1$ ) et sont reliées simplement au temps de relaxation $T_{1}$. En effet, si l'on définit :

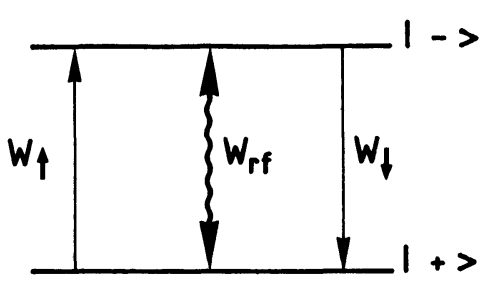

Fig. 6. - Transitions de relaxation et de radio-fréquence induites entre deux niveaux électroniques.

[Relaxation and radio-frequency transitions induced between two electronic levels.] 
$\Delta p=p_{+}-p_{-}$et $\Delta p^{\mathrm{B}}=p_{+}^{\mathrm{B}}-p_{-}^{\mathrm{B}}$, on montre facilement que l'ensemble des équations (72) est équivalent à :

$$
\left.\begin{array}{rl}
p_{+}+p_{-} & =p_{+}^{\mathrm{B}}+p_{-}^{\mathrm{B}} \\
\frac{\mathrm{d} \Delta p}{\mathrm{~d} t} & =-\left(W_{\uparrow}+W_{\downarrow}\right)\left(\Delta p-\Delta p^{\mathrm{B}}\right)- \\
- & 2 W_{\mathrm{rf}} \Delta p=-\frac{\Delta p-\Delta p^{\mathrm{B}}}{T_{1}}-2 W_{\mathrm{rf}} \Delta p
\end{array}\right\}
$$

avec :

$$
\frac{1}{T_{1}}=W_{\uparrow}+W_{\downarrow},
$$

puisque (73) se réduit à l'équation (31) en l'absence de radiofréquence $\left(W_{\mathrm{rf}}=0\right)$.

En régime stationnaire, $\mathrm{d} \Delta p / \mathrm{d} t=0$ et la résolution de (73) conduit à :

$$
\Delta p=\Delta p_{\mathrm{B}} \frac{1}{1+2 T_{1} W_{\mathrm{rf}}} .
$$

La puissance absorbée à la résonance vaut alors :

$$
S\left(\omega_{0}, W_{\mathrm{rf}}\right)=2 \pi h^{2} g^{2} \mu_{\mathrm{B}}^{2} T_{2}^{\prime} \omega_{0} \Delta p_{\mathrm{B}} \frac{1}{1+2 T_{1} W_{\mathrm{rf}}},
$$

et la mesure de la variation de $S$ en fonction de $W_{\mathrm{rf}}$ donne accès à $T_{1}$.

Par les méthodes d'analyse en temps ou de l'onde continue, il est possible de mesurer des temps de relaxation supérieurs à $1 \mu \mathrm{s}$, c'est-à-dire de l'ordre de grandeur des temps de relaxation spin-phonons à la température de l'hélium liquide. Ces méthodes ont donc été largement utilisées pour étudier la dynamique des impuretés magnétiques dans les isolants à très basse température. Une bonne revue sur le sujet est constituée par la référence [9].

Les temps de relaxation $T_{1}$ et $T_{2}$, associés respectivement à la variation temporelle de $\left\langle S_{\mathrm{x}}\right\rangle$ et $\left\langle S_{+}\right\rangle$ (ou $\left\langle S_{-}\right\rangle$), ont été introduits initialement de manière phénoménologique par Bloch [28] pour décrire l'évolution de l'aimantation $M$ en R.M.N. ou en R.P.E. :

$$
\left\{\begin{array}{l}
\frac{\mathrm{d} M_{\mathrm{z}}}{\mathrm{d} t}=-\frac{M_{\mathrm{z}}-M_{\mathrm{z}}^{\mathrm{B}}}{T_{1}} \\
\frac{\mathrm{d} M_{x, y}}{\mathrm{~d} t}=-\frac{M_{x, y}}{T_{2}}
\end{array}\right.
$$

(équations de Bloch).

Dans les systèmes concentrés en ions magnétiques identiques, le rayonnement électromagnétique est couplé en fait au moment magnétique électronique total : $\mathbf{M}=\sum_{r_{i}} \mathbf{m}\left(\mathbf{r}_{i}\right)$, où $\mathbf{r}_{i}$ est le rayon vecteur de la position d'un ion individuel, et le signal observé en R.P.E. (resp. R.M.N.) est lié à l'aimantation totale électronique (resp. nucléaire). La situation en spectroscopie Mössbauer est différente de ce point de vue, car la mesure est « locale », le signal observé étant la somme des signaux individuels des noyaux.

En R.P.E., le temps de relaxation $T_{1}$ décrit donc le retour vers l'équilibre de l'aimantation totale $M_{\mathbf{z}}$.
Les mécanismes de relaxation qui conservent $M_{z}$, tel le renversement simultané de deux spins identiques ("flip-flop ") induit par le terme $S_{i}^{+} S_{j}^{-}$de l'échange isotrope, ne contribuent pas à $T_{1}$, qui est déterminé uniquement par la relaxation "spin-réseau ». Par contre, ces processus "flip-flop " contribuent à la décroissance des composantes transverses $M_{x}$ et $M_{y}$, en un temps $T_{\mathrm{ss}}$ qui est en général beaucoup plus court que $T_{1}\left(T_{\mathrm{ss}} \sim 10^{-8} \mathrm{~s}-10^{-9} \mathrm{~s}\right)$. Le temps $T_{2}$ est alors souvent appelé " temps de relaxation spin-spin ", car $T_{2} \simeq T_{\mathrm{ss}}$. Donc, dans les composés concentrés en ions magnétiques : $T_{2} \ll T_{1}$. En spectroscopie Mössbauer, sans champ magnétique appliqué, la distinction entre $T_{1}$ et $T_{2}$ n'a plus lieu d'être : on peut alors étudier la relaxation " spin-spin " à champ nul, ce qui implique un traitement assez différent de celui utilisé en R.P.E. (cf. § 6).

Dans les cas où le système étudié est une impureté paramagnétique diluée, les deux temps $T_{1}$ et $T_{2}$ relèvent du même mécanisme de relaxation (phonons dans les isolants, électrons de conduction dans les métaux) et sont du même ordre de grandeur, comme le montrent les expressions (30) et (47). Lorsque l'interaction de relaxation est isotrope, comme par exemple dans les alliages dilués en site cubique, et à haute température $\left(k_{\mathrm{B}} T \gg \omega_{0}\right)$, les deux temps $T_{1}$ et $T_{2}$ sont égaux [29], comme il a déjà été signalé au § 3.2.

c) Dans les solides, les neutrons interagissent avec les noyaux, les moments magnétiques, les vibrations $\mathrm{du}$ réseau, etc... La spectroscopie de diffusion inélastique des neutrons permet d'obtenir soit les courbes de dispersion des énergies d'excitation du solide en fonction du transfert d'impulsion $\mathbf{q}$, soit, à $q$ fixe, la section efficace de diffusion en fonction de la fréquence $\omega$.

Par comparaison avec une substance isostructurale, mais diamagnétique, il est en général possible d'isoler la contribution à la section efficace due à la diffusion inélastique des neutrons par les moments électroniques du corps étudié. On peut montrer [31] que la section efficace différentielle $\mathrm{d}^{2} \sigma / \mathrm{d} \omega \mathrm{d} \Omega$ de diffusion avec gain ou perte d'énergie $\omega$ par le neutron s'exprime par :

$$
\frac{\mathrm{d}^{2} \sigma}{\mathrm{d} \omega \mathrm{d} \Omega} \propto \operatorname{Re} \int_{0}^{\infty} \mathrm{d} t \mathrm{e}^{i \omega t}\left\langle m_{\mathrm{z}}(\mathbf{q}, t) m_{\mathrm{z}}(-\mathbf{q})\right\rangle,
$$

dans un système à symétrie cubique et en choisissant l'axe $\mathrm{O} z$ perpendiculaire au transfert d'impulsion $\mathbf{q}$, qui est fixé dans l'expérience. L'opérateur $m_{z}(q)$ est la composante de Fourier spatiale de la densité d'aimantation $m_{\mathrm{z}}(\mathbf{r})$ associée à un ion paramagnétique diffuseur :

$$
m_{z}(\mathbf{q})=\int \mathrm{d}^{3} r \mathrm{e}^{-i q r} m_{z}(\mathbf{r}) .
$$

Lorsque les électrons du centre diffuseur sont localisés, comme dans le cas des terres rares (rayon ionique : 
$r_{\mathrm{f}} \leqslant 0,5 \AA$ ), l'opérateur $m_{\mathrm{z}}(\mathbf{q})$ peut s'écrire, pour les grandes valeurs de $q\left(q>1 / r_{\mathrm{f}}\right)$ :

$$
m_{\mathrm{z}}(\mathbf{q}) \simeq g_{J} \mu_{\mathrm{B}} J_{\mathrm{z}} F(\mathbf{q}),
$$

où $F(q)$ est le facteur de forme magnétique de l'ion, et $\mathbf{J}$ le moment angulaire de la terre rare. La section efficace de diffusion avec perte d'énergie du neutron devient alors simplement, en introduisant la largeur expérimentale $\Gamma$ :

$$
\begin{array}{r}
\frac{\mathrm{d}^{2} \sigma}{\mathrm{d} \Omega \mathrm{d} \omega} \propto|F(q)|^{2} \operatorname{Re} \int_{0}^{\infty} \mathrm{d} t \mathrm{e}^{i \omega t-\Gamma t / 2}\left\langle J_{\mathrm{z}}(t) J_{\mathrm{z}}\right\rangle= \\
=|F(q)|^{2} P^{\mathrm{a}}(\omega),
\end{array}
$$

où $P^{\mathrm{a}}(\omega)$ est la probabilité d'absorption définie par (43). Sous une autre forme :

$$
\frac{\mathrm{d}^{2} \sigma}{\mathrm{d} \omega \mathrm{d} \Omega} \propto \frac{\chi^{\prime \prime}(\omega)}{1-\mathrm{e}^{-\omega / k_{B} T}}=\frac{\operatorname{Re}[j(\omega)]}{1+\mathrm{e}^{-\omega / k_{B} T}} .
$$

Dans les composés intermétalliques de terre rare, les niveaux de champ cristallin sont séparés par des énergies de l'ordre de $100 \mathrm{~K}$. A l'aide de faisceaux monochromatiques de neutrons de quelques dizaines de $\mathrm{meV}(1 \mathrm{meV} \simeq 11,6 \mathrm{~K})$, la détermination de la structure de champ cristallin de ces composés a fait l'objet de nombreux travaux [39]. Lorsque les effets de relaxation sont négligeables, la section efficace inélastique «normalisée » :

$$
\left(1+\mathrm{e}^{-\omega / k_{\mathbf{B}} T}\right) \frac{\mathrm{d}^{2} \sigma}{\mathrm{d} \omega \mathrm{d} \Omega}=\operatorname{Re}[j(\omega)]
$$

se compose de raies lorentziennes, de largeur $\Gamma$, situées aux diverses énergies de transition entre les niveaux de champ cristallin (les expériences sont réalisées en l'absence de champ magnétique appliqué, en zone paramagnétique).

Si l'on considère le doublet fondamental en symétrie cubique d'un ion de Kramers seul peuplé à basse température $\left(k_{\mathrm{B}} T \ll \Delta_{\mathrm{cc}}\right.$ : énergie du premier doublet excité), la forme de raie, lorsque l'énergie $E_{0}$ des neutrons incidents est inférieure à $\Delta_{\mathrm{cc}}$, se réduit à la seule raie "quasi élastique" centrée en $\omega=0$, correspondant aux transitions quasi élastiques induites entre les deux états dégénérés du doublet. En présence de relaxation, le calcul de $\operatorname{Re}[j(\omega)]$ est analogue à celui effectué dans le $\S 4.1 a$, et on obtient :

$$
\begin{aligned}
& \operatorname{Re}[j(\omega)]= \\
& =\frac{4}{\pi g^{2}}\left|\left\langle+\left|J_{z}\right|+\right\rangle\right|^{2} \frac{\Gamma / 2+1 / T_{2}}{\left(\Gamma / 2+\frac{1}{T_{2}}\right)^{2}+\omega^{2}},
\end{aligned}
$$

car pour un doublet dégénéré en symétrie cubique : $1 / T_{1}=1 / T_{2}=2 W, W$ étant la fréquence de relaxation entre les deux états du doublet.

Si l'on augmente l'énergie incidente des neutrons de manière que : $E_{0}>\Delta_{\text {cc }}$, les raies inélastiques appa- raissent, leur élargissement dynamique dépendant cette fois des fréquences de relaxation entre le doublet fondamental et le niveau excité. Cependant, lorsque la température augmente, la relaxation s'accélère, et les raies individuelles se fondent progressivement en une seule raie quasi élastique. Cet " effacement" de la structure du spectre sera discuté dans le $\$ 4.3$.

Dans la plupart des expériences, la résolution des spectromètres en « temps de vol " atteint $E_{0} / 20$ pour $E_{0}<5 \mathrm{meV}$, et $E_{0} / 10$ pour $E_{0} \simeq 80 \mathrm{meV}$. Si l'on prend une résolution moyenne de $1 \mathrm{meV}$, la largeur statique $\Gamma$ des raies vaudra : $\Gamma \sim 10^{11} \mathrm{~Hz}$. A basse température, les effets d'élargissement dynamique, plus faibles $\left(W \sim 10^{9}-10^{10} \mathrm{~Hz}\right.$ pour la relaxation spin-spin) sont donc en fait inobservables. Cependant, actuellement, avec la résolution accrue des spectromètres, il devient possible d'étudier la relaxation paramagnétique dans certains cas favorables à basse température, dans les composés intermétalliques de terre rare [33]. Sur la figure 7, on peut voir les spectres de perte d'énergie de neutrons incidents de $50,4 \mathrm{meV}$ dans les composés $\mathrm{CeAg}$ et $\mathrm{LaAg}$. $\mathrm{La}$ raie de champ cristallin dans $\mathrm{CeAg}$, située en $E_{0} \simeq-24 \mathrm{meV}$, s'élargit progressivement, lorsque la température augmente, sous l'effet de la relaxation par interaction d'échange avec les électrons itinérants.

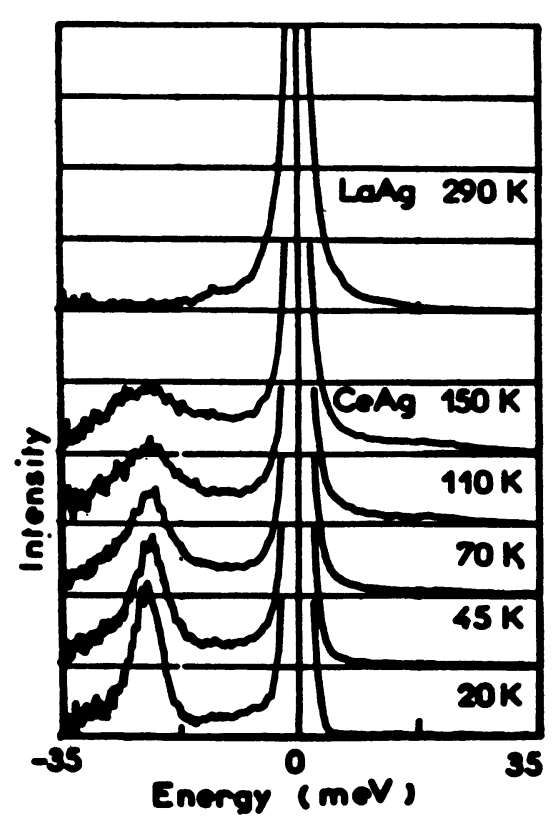

Fig. 7. - (D'après [33b]) Sections efficaces normalisées de diffusion des neutrons à différentes températures dans $\mathrm{CeAg}$ et le composé isostructural non magnétique $\mathrm{LaAg}$ (ces données sont la somme de détecteurs placés à des angles de diffusion compris entre $2,25^{\circ}$ et $20,25^{\circ}$ ).

[(After [33b]) Normalized cross-sections of neutron scattering at different temperatures in $\mathrm{CeAg}$ and in the isostructural non-magnetic compound $\mathrm{LaAg}$ (these data are the sum of detectors placed at diffusion angles between $2.25^{\circ}$ and $\left.20.25^{\circ}\right)$.] 
Cette technique, pour l'instant peu employée dans l'étude de la relaxation paramagnétique, est certainement appelée à jouer un rôle plus important dans le domaine de température $T \gtrsim 100 \mathrm{~K}$, inaccessible à l'aide, par exemple, de la R.P.E. (sauf pour l'ion $\mathrm{S} \mathrm{Gd}^{3+}$ ).

Signalons enfin que dans certains composés intermétalliques de cérium ou d'ytterbium on n'observe qu'une seule raie "quasi élastique", très large $(\Gamma \simeq 10 \mathrm{meV})$ [32], et pas de structure de champ cristallin. Dans ces composés, dits "à valence intermédiaire ", existe un couplage anormalement fort entre les électrons $4 \mathrm{f}$ de la terre rare et les électrons de conduction, qui induit des fluctuations très rapides du moment électronique et "efface" l'interaction de champ cristallin. La spectroscopie inélastique de neutrons a permis de mesurer ces fréquences de fluctuation, la largeur dynamique de $10 \mathrm{meV}$ de la raie quasi élastique correspondant à une fréquence d'environ $10^{13} \mathrm{~Hz}$.

d) La spectroscopie optique concerne les transitions entre l'état fondamental de l'ion et un étát excité de champ cristallin (pour les ions 3d) ou de spin orbite (pour les ions terre rare). Les photons optiques ayant une énergie de l'ordre de $10^{14} \mathrm{~Hz}$, la largeur intrinsèque $\Gamma^{\text {in }}$ est importante, et donne lieu à des " temps de vie radiatifs " des niveaux excités qui, pour les terres rares, sont de l'ordre de la milliseconde [37]. Sans nous étendre sur le sujet, faute de place et de compétence de l'auteur, signalons que la spectroscopie optique a été utilisée pour mesurer les fréquences de relaxation par les phonons d'impuretés de transition ou de terres rares dans des matrices isolantes. Les deux types de méthodes de mesure sont employés : analyse en temps de la fluorescence issue de niveaux excités [35], ou analyse des largeurs de raie homogènes des transitions d'absorption ou d'émission [36].

Ce type d'études s'est beaucoup développé avec l'apparition des lasers, et avec l'amélioration progressive de leurs performances (bande d'excitation étroite, impulsions très courtes, etc...). Pour plus de détails, le lecteur est renvoyé à la référence [37].

4. 2 LES MESURES " HYPERFINES ». - Les transitions entre les niveaux nucléaires peuvent être utilisées en spectroscopie : l'effet Mössbauer et les mesures de corrélations angulaires mettent en jeu les transitions entre l'état fondamental du noyau et les états excités séparés par quelques dizaines de $\mathrm{keV}\left(10^{18} \mathrm{~Hz}\right)$. La résonance magnétique nucléaire observe les transitions entre les niveaux Zeeman, séparés par un champ magnétique, de l'état fondamental nucléaire, à l'aide de radiation de radiofréquence de quelques $\mathrm{MHz}$.

Les interactions hyperfines dans les solides sont de deux types principaux : les interactions magnétiques entre le spin $I$ du noyau et les moments magnétiques des électrons, et les interactions quadrupolaires électriques entre le moment quadrupolaire du noyau (pour $I>1 / 2$ ) et le gradient de champ électrique créé par les couches électroniques de l'ion et les autres charges ioniques présentes dans le réseau.
Les niveaux nucléaires sont perturbés par le couplage hyperfin, et les fluctuations électroniques se reflètent sur les spectres observés en spectroscopie Mössbauer et en corrélations angulaires, où l'on mesure en principe directement la fréquence de relaxation électronique, et sur la fréquence de relaxation nucléaire mesurée par R.M.N.

a) La spectroscopie Mössbauer met à profit l'absorption ou l'émission " sans recul » de photons $\gamma$ par une fraction des noyaux dans un solide, qui transitent entre le premier état excité, de temps de vie $\tau_{n}$, et l'état fondamental. La raie Mössbauer possède une largeur " naturelle " $\Gamma_{\text {in }}=\hbar / \tau_{\mathrm{n}}$, qui est de l'ordre de $10 \mathrm{MHz}$ $\left(1,16 \mathrm{MHz}\right.$ pour ${ }^{57} \mathrm{Fe}$ et environ $50 \mathrm{MHz}$ pour les terres rares). Les énergies hyperfines $\omega_{\text {hpf }}$ valant entre $50 \mathrm{MHz}$ et $1 \mathrm{GHz}$, les «bons " isotopes Mössbauer $\left(\Gamma_{\text {in }}<\omega_{\text {hpf }}\right)$ permettent l'observation de spectres hyperfins résolus, sans qu'il soit nécessaire d'appliquer un champ magnétique.

Dans l'immense majorité des expériences, on a recours à l'analyse spectrale en énergie dans une géométrie de transmission. En spectrométrie d'absorption, la source, contenant le parent radioactif de l'isotope étudié, est choisie de telle manière que son spectre d'émission soit monochromatique et le plus fin possible. Le balayage est effectué par effet Doppler, la source étant montée sur un vibreur électromagnétique. Il est également possible d'observer des spectres d'émission, auquel cas c'est un absorbeur monochromatique animé de mouvement qui analyse la structure hyperfine du spectre de la source. La spectrométrie d'émission doit être utilisée dans l'étude d'impuretés, lorsque le spectre d'absorption correspondant serait trop faible pour être détecté.

En spectrométrie d'absorption, on observe le faisceau $\gamma$ transmis à travers un absorbeur d'épaisseur $\underline{d}$. La probabilité d'absorption résonnante d'un photon d'énergie $\omega$ par un noyau vaut :

$$
\sigma(\omega)=f_{\mathrm{a}} \sigma_{0} \frac{\Gamma_{\mathrm{in}}}{2} P^{\mathrm{a}}(\omega),
$$

où $f_{\mathrm{a}}$ est la probabilité d'absorption sans recul, $\sigma_{0}$ la constante nucléaire de section efficace, et $P^{\mathbf{a}}(\omega)$ est défini par l'expression (43). Après la traversée de l'absorbeur, l'intensité du faisceau incident $I(\omega)$ est réduite d'un facteur : $\mathrm{e}^{-n \sigma(\omega) d}$, où $n$ est le nombre de noyaux résonnants par unité de volume de l'absorbeur. Le signal observé, qui est l'absorption résonnante, est donc proportionnel à :

$$
S(\omega)=1-\mathrm{e}^{-n \sigma(\omega) d}=1-\mathrm{e}^{-\Gamma_{\text {in }} T_{A} P^{a}(\omega)}
$$

où l'on a défini l'épaisseur effective : $T_{A}=n f_{\mathrm{a}} \sigma_{0} d$.

Dans la limite d'un absorbeur "mince " $\left(T_{A} \ll 1\right)$, le signal d'absorption $S(\omega)$ est donc directement proportionnel à $P^{\mathrm{a}}(\omega)$, c'est-à-dire comme :

$$
\begin{aligned}
P^{\mathrm{e}}(\omega) & =\mathrm{e}^{-\omega / k_{\mathrm{B}} T} P^{\mathrm{a}}(\omega) \simeq 0 \quad\left(\omega / k_{\mathrm{B}} \sim 10^{8} \mathrm{~K}\right) \\
S(\omega) & =\frac{\Gamma_{\text {in }}}{2} T_{\mathrm{a}} \operatorname{Re}[j(\omega)] .
\end{aligned}
$$


En l'absence de relaxation, le spectre est formé de raies lorentziennes, centrées sur les énergies de transition entre niveaux hyperfins, et de largeur statique $\Gamma_{\text {exp }}$. Une situation couramment rencontrée est celle où les niveaux électroniques sont quantifiés par une interaction (champ cristallin ou énergie Zeeman) bien supérieure à l'énergie hyperfine. Dans ce cas, chaque fonction d'onde électronique correspond à une valeur du champ hyperfin ou du gradient de champ électrique vu par le noyau. Considérons par exemple un doublet de Kramers sur un site à symétrie cubique, en présence d'un champ appliqué ou moléculaire $H_{0}$, tel que :

$$
g \mu_{\mathrm{B}} H_{0} \gg A_{\mathrm{hpf}},
$$

où $A_{\text {hpf }}$ est la constante de l'interaction hyperfine :

$$
\mathscr{H}_{\mathrm{hpf}}=A_{\mathrm{hpf}} \mathrm{I} . \mathrm{S} \quad \text { (I est le spin du noyau). }
$$

Cette interaction peut prendre les deux valeurs : $A_{\text {hpf }} I_{z}\left\langle+\left|S_{z}\right|+\right\rangle$ et $A_{\text {hpf }} I_{z}\left\langle-\left|S_{z}\right|-\right\rangle$, correspondant à deux champs hyperfins opposés :

$$
\left\langle\mathscr{H}_{\mathrm{hpf}}\right\rangle=-g_{\mathrm{n}} \mu_{\mathrm{n}} I_{\mathrm{z}} H_{\mathrm{hpf}},
$$

avec :

$$
H_{\mathrm{hpf}}= \pm \frac{A_{\mathrm{hpf}}}{2 g_{\mathrm{n}} \mu_{\mathrm{n}}}
$$

$g_{\mathrm{n}}$ et $\mu_{\mathrm{n}}$ étant respectivement la constante gyromagnétique du noyau (dans l'état fondamental ou excité) et le magnéton de Bohr nucléaire.

L'effet de la relaxation paramagnétique, en induisant des transitions entre $|+\rangle$ et $|-\rangle$, revient à faire varier le champ hyperfin vu par le noyau entre deux valeurs opposées, c'est-à-dire en fait à moduler la valeur de l'énergie d'une transition donnée, comme il est facile de s'en rendre compte sur la figure 8. Dans l'exemple donné, l'énergie de la transition d'absorp-

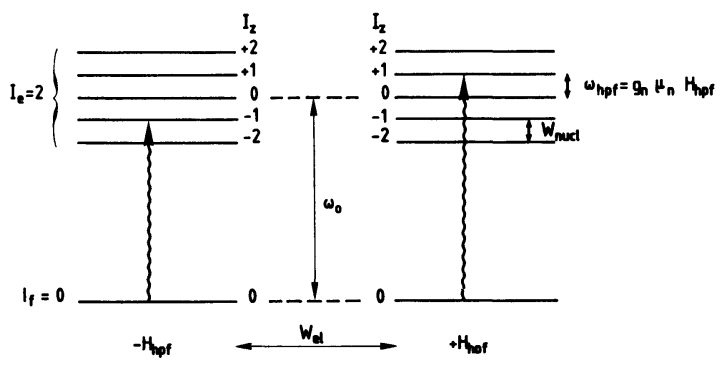

Fig. 8. - Schéma de niveaux hyperfins de ${ }^{170} \mathrm{Yb}\left(g_{\mathrm{n}}>0\right)$ en présence de relaxation électronique (fréquence $W_{\mathrm{et} 1}$ ) entre deux états correspondant à des champs hyperfins opposés et de même grandeur. Les transitions Mössbauer d'absorption $\Delta I_{z}=-1$ sont représentées par : $\hat{\text {. }}$.

[Hyperfine level scheme of ${ }^{170} \mathrm{Yb}\left(g_{\mathrm{n}}>0\right)$ in the presence of electronic relaxation (rate $W_{\mathrm{el}}$ ) between two states corresponding to equal and opposite hyperfine fields. The absorption Mössbauer transitions with $\Delta I_{z}=-1$ are represented by $: \hat{\xi}$.] tion $\Delta I_{\mathrm{z}}=-1$ fluctue entre les valeurs $E_{0}-\omega_{\mathrm{hpf}}$ et $E_{0}+\omega_{\mathrm{hpf}}$, à la fréquence de relaxation électronique $W_{\text {el }}$. L'effet de la relaxation sur le spectre ne peut pas se réduire à un simple élargissement dynamique de la raie, proportionnel à la fréquence de relaxation mais relève du phénomène de "rétrécissement par le mouvement ", dont nous parlerons au $\S$ 4.3. Dans l'exemple de la figure 8 , le spectre hyperfin magnétique à cinq raies $\left(2 I_{\mathrm{e}}+1=5\right)$ se brouille progressivement lorsque la fréquence de relaxation $W_{\mathrm{el}}$ augmente, la structure étant "effacée " lorsque : $W_{\mathrm{el}} \sim \omega_{\mathrm{hpf}}$, où $\omega_{\text {hpf }}$ est l'écart en fréquence entre deux raies. A ce moment, la forme générale du spectre est très sensible aux variations de $W_{\mathrm{el}}$; le domaine de fréquences de relaxation que l'on peut mesurer par l'analyse en énergie est donc en gros :

$$
\frac{\omega_{\mathrm{hpf}}}{10} \lesssim W_{\mathrm{el}} \lesssim 10 \omega_{\mathrm{hpf}} .
$$

Cette "fenêtre " est centrée sur $50 \mathrm{MHz}$ pour l'isotope ${ }^{57} \mathrm{Fe}$, et sur $1 \mathrm{GHz}$ pour les isctopes de terre rare $\left({ }^{166} \mathrm{Er},{ }^{170} \mathrm{Yb}\right.$, etc...).

Lorsque la relaxation devient très " rapide", nous avons vu que la raie en R.P.E. s'élargit proportionnellement à $1 / T_{2}$, sous l'influence des transitions de relaxation induites entre les deux niveaux Zeeman. $\mathrm{Au}$ contraire, en spectroscopie Mössbauer, la relaxation électronique ne peut pas coupler les deux états (ici nucléaires) entre lesquels ont lieu les transitions spectroscopiques. Il en résulte que le rétrécissement par le mouvement est complet, le spectre s'affinant de plus en plus lorsque la fréquence de relaxation augmente (cf. § 4.3).

Remarquons que les mécanismes de relaxation nucléaire (par les phonons ou les électrons de conduction) peuvent également contribuer à la modulation des énergies de transition. Sur la figure 8 , on a indiqué une transition de relaxation nucléaire possible $\left(W_{\text {nucl }}\right)$ entre deux états $\left(\left|\Delta I_{z}\right|=1\right)$. Cependant, ces fréquences $W_{\text {nucl }}$ sont très faibles $\left(W_{\text {nucl }} \lesssim 1 \mathrm{kHz}\right.$ à température ambiante), et donc : $W_{\text {nucl }} \ll \omega_{\text {hpf }}$. La relaxation nucléaire n'a donc aucune influence sur les spectres Mössbauer.

La plupart des études de relaxation ont été réalisées à l'aide des isotopes ${ }^{57} \mathrm{Fe}$ (ion $\mathrm{Fe}^{3+}$ ), ${ }^{161} \mathrm{Dy}$ (ion $\mathrm{Dy}^{3+}$ ) et ${ }^{170} \mathrm{Yb}$ (ion $\mathrm{Yb}^{3+}$ ). L'effet Mössbauer, ne nécessitant pas l'application de champ magnétique, permet l'observation des phénomènes de relaxation à champ nul, contrairement à la R.P.E.

La référence [40] constitue la première revue sur la relaxation spin-spin et spin-réseau mesurée par effet Mössbauer. Plus récemment, une revue consacrée exclusivement aux phénomènes de relaxation de $\mathrm{Fe}^{3+}$ est parue [41b]. Quant aux calculs théoriques de forme de raie, ils ont donné lieu à une abondante littérature, dont on trouvera une liste dans la référence [42].

La figure $9[41 a]$ illustre les effets spectaculaires de la relaxation dans un composé isolant contenant des ions $\mathrm{Fe}^{3+}$ : la fréquence de relaxation spin-phonons 


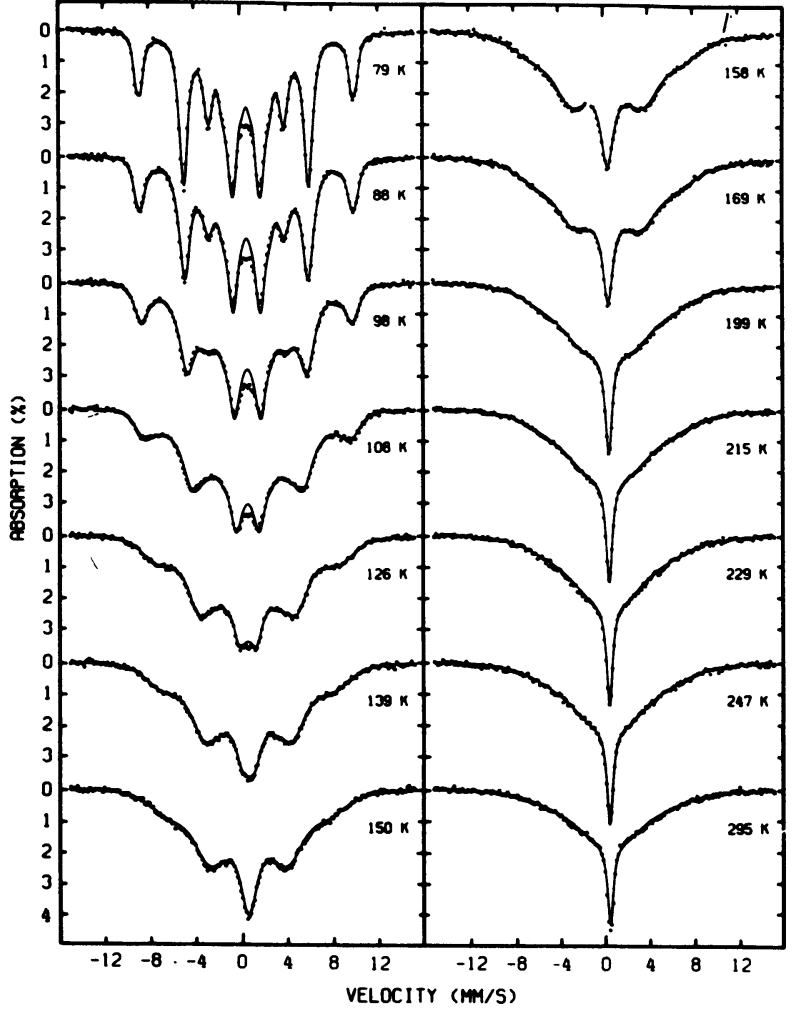

Fig. 9. - (D'après [41a]) Spectres Mössbauer des ions ${ }^{57} \mathrm{Fe}^{3+}$ substitués dans $\mathrm{NH}_{4} \mathrm{Al}\left(\mathrm{SO}_{4}\right)_{2} .12 \mathrm{H}_{2} \mathrm{O}$ à diverses températures sous un champ magnétique de $12,3 \mathrm{kG}$ appliqué perpendiculairement à la direction de propagation des $\gamma$.

[(After [41a]) Mössbauer spectra of ${ }^{57} \mathrm{Fe}^{3+}$ ions substituted in $\mathrm{NH}_{4} \mathrm{Al}\left(\mathrm{SO}_{4}\right)_{2} .12 \mathrm{H}_{2} \mathrm{O}$ at various temperatures in a $12.3 \mathrm{kG}$ magnetic field applied perpendicularly to the direction of propagation of the $\gamma$-rays.]

vaut environ $1 \mathrm{MHz}$ à $T=79 \mathrm{~K}$, et augmente jusqu'à $100 \mathrm{MHz}$ vers $T=250 \mathrm{~K}$. Ces valeurs relativement basses viennent du fait que l'ion $\mathrm{Fe}^{3+}$ est un ion sans moment orbital $(L=0$, ion $S)$, et que le couplage spin-phonons n'apparaît qu'au second ordre en perturbation.

La spectrométrie Mössbauer d'émission consiste à observer l'émission spontanée d'un photon $\gamma$ à partir du niveau nucléaire excité ; ce dernier est peuplé à la suite d'une cascade radioactive issue du noyau parent de l'isotope considéré. On peut alors analyser en énergie le rayonnement émis, ce qui a été mis à profit pour mesurer les fréquences de relaxation d'impuretés de terre rare $\left(\mathrm{Yb}^{3+}, \mathrm{Er}^{3+}\right)$ dans quelques alliages dilués [43].

La figure 10, tirée de la référence [43b], montre l'évolution du spectre de ${ }^{170} \mathrm{Yb}^{3+}$ dans l'or sous l'influence de la relaxation par l'interaction d'échange avec les électrons de conduction. La fréquence de relaxation du moment magnétique de $\mathrm{Yb}^{3+}$, «lente » à $T=1,27 \mathrm{~K}(W \simeq 200 \mathrm{MHz})$, atteint $2 \mathrm{GHz}$ vers $T=20 \mathrm{~K}$.

Signalons enfin que l'apparition d'états métastables (de charge, de champ cristallin, etc...) peuplés hors

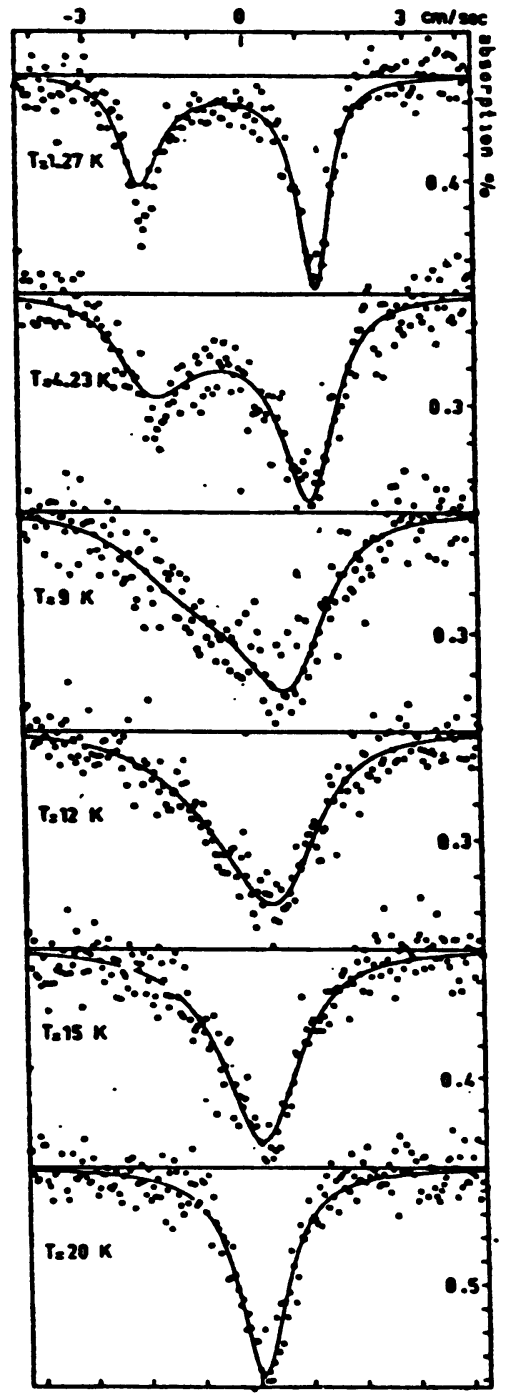

Fig. 10. - (D'après [43]) Spectres Mössbauer d'émission de ${ }^{170} \mathrm{Yb}^{3+}$ dilué dans $\mathrm{Au}$.

[(After [43]) Mössbauer emission spectra of ${ }^{170} \mathrm{Yb}^{3+}$ diluted in $\mathrm{Au}$.]

équilibre thermique à l'issue de la cascade nucléaire dans les sources Mössbauer, peut être observée en spectrométrie d'émission, lorsque le temps de vie $\tau$ de ces états est comparable au temps de vie $\tau_{\mathrm{n}}$ du niveau nucléaire excité. En particulier, le temps de relaxation paramagnétique $T_{1}$ de l'impureté peut être mesuré, lorsque les états métastables peuplés sont des niveaux hyperfins ou de champ cristallin, par des méthodes analogues à l'analyse en temps de la R.P.E. ou de la R.M.N. Ces effets sont décrits en détail dans l'article de P. Imbert [38].

b) Dans les mesures des corrélations angulaires perturbées $\gamma-\gamma$, on observe les deux rayonnements $\gamma$ successifs émis lors d'une cascade radioactive à partir d'un niveau nucléaire initial vers le niveau fondamental. La décroissance $\gamma_{1}$ à partir de ce niveau initial peuple sélectivement les sous-niveaux nucléaires du niveau intermédiaire, les laissant hors équilibre ther- 
mique à cet instant choisi comme instant $t=0$. Dans ces expériences, réalisées à haute température, l'équilibre de Boltzmann correspond à l'équipopulation des sous-niveaux nucléaires.

L'instant d'émission du second photon $\gamma_{2}$ (émis à partir du niveau intermédiaire) est déterminé par des méthodes de coïncidence par rapport à l'émission du photon initial $\gamma_{1}$.

En choisissant la direction d'émission de $\gamma_{1}$ comme axe origine, la distribution angulaire de $\gamma_{2}$ peut s'écrire [4], si l'on suppose que les états nucléaires intermédiaires sont stationnaires (absence de perturbation statique ou dépendant du temps) :

$$
w(\theta) \simeq 1+A_{2} P_{2}(\cos \theta),
$$

où $A_{2}$ est un coefficient dépendant des spins nucléaires des niveaux en jeu et : $P_{2}(\cos \theta)=1+3 \cos 2 \theta$.

En présence d'une perturbation statique des niveaux nucléaires, la corrélation angulaire est perturbée et devient dépendante du temps. Par exemple, l'application d'un champ magnétique perpendiculairement au plan de l'expérience "fait tourner" la dépendance angulaire, sans détruire la corrélation :

$$
w(\theta, t)=1+A_{2} P_{2}\left[\cos \left(\theta-\omega_{\mathrm{L}} t\right)\right],
$$

où $\omega_{\mathbf{L}}$ est la fréquence de Larmor nucléaire.

Par contre, dans le cas d'une perturbation induisant des transitions entre les sous-niveaux nucléaires de l'état intermédiaire, la corrélation est atténuée d'un facteur $G(t)$, qui s'annule aux temps très longs :

$$
w(\theta, t)=1+A_{2} G(t) P_{2}(\cos \theta) .
$$

En effet, lorsque la relaxation au sein des sous-niveaux nucléaires a rétabli l'équipopulation, la distribution des photons émis est isotrope $(w(\theta, \infty)=1)$ et la corrélation est détruite.

L'observation de l'évolution temporelle de $w(\theta, t)$ à $\theta$ fixe (corrélations angulaires différentielles ou " TDPAC ») peut alors permettre d'obtenir des informations sur la relaxation paramagnétique d'une impureté, lorsque la relaxation nucléaire est induite par l'interaction hyperfine. Cette possibilité a été discutée de manière complète dans la référence $[15 b]$, où il est montré que le facteur $G(t)$ décrit en fait le retour à l'équilibre thermique des composantes d'un tenseur "quadrupolaire " nucléaire $Q_{2}$; en particulier, lorsque le temps de relaxation électronique est très rapide, la variation temporelle de $: Q_{\mathrm{z}}^{2}=\frac{1}{\sqrt{6}} \times$ $\left\langle 3 I_{z}^{2}-I(I+1)\right\rangle$ est décrite par :

$$
G(t)=\mathrm{e}^{-3 t / T_{1 n}}
$$

où $T_{1 \mathrm{n}}$ est le temps de relaxation longitudinal nucléaire donné par [4] :

$$
\frac{1}{T_{1 \mathrm{n}}}=\frac{2}{3} A^{2} S(S+1) T_{1}
$$

$T_{1}$ étant le temps de relaxation électronique longitudinal et $\boldsymbol{A}$ la constante de l'interaction hyperfine.

Si l'on ne fait pas d'analyse en temps, on mesure la corrélation :

$$
w_{i}(\theta)=\frac{1}{\tau_{\mathrm{n}}} \int_{0}^{\infty} \mathrm{e}^{-t / \tau_{\mathrm{n}}} w(\theta, t) \mathrm{d} t,
$$

où $\tau_{\mathfrak{n}}$ est le temps de vie de l'état nucléaire intermédiaire. On parle alors de corrélations angulaires intégrales ou "TIPAC ». Dans le cas d'une décroissance exponentielle de $G(t)$ (expression (89)) on a :

$$
w_{i}(\theta)=1+A_{2} P_{2}(\cos \theta) \frac{1}{1+\frac{3 \tau_{\mathrm{n}}}{T_{1 \mathrm{n}}}} .
$$

Les corrélations angulaires intégrales permettent donc de mesurer $T_{1 \mathrm{n}}$ si celui-ci est du même ordre de grandeur que $\tau_{\mathbf{n}}$ (cf. également la référence [38]).

Récemment, la technique de TDPAC a été l'objet d'un regain d'intérêt, à l'aide d'un dispositif expérimental comportant un champ magnétique pulsé [44] et permettant de mesurer la quantité :

$$
R(t)=A_{2} \sin \left(2 \omega_{\mathrm{hpf}} t\right) \cdot \mathrm{e}^{-3 t / T_{\mathrm{ln}}},
$$

où $\omega_{\text {hpf }}$ est la fréquence hyperfine. Les mesures [45], effectuées sur des isotopes de $\mathrm{Eu}$ et de $\mathrm{Ce}$ à longue durée de vie dans des matrices métalliques simples, permettent d'obtenir le champ hyperfin (période de $R(t))$ et $T_{1 \mathrm{n}}$ (enveloppe exponentielle), comme le montre la figure $11[45 b]$. On peut alors en déduire le

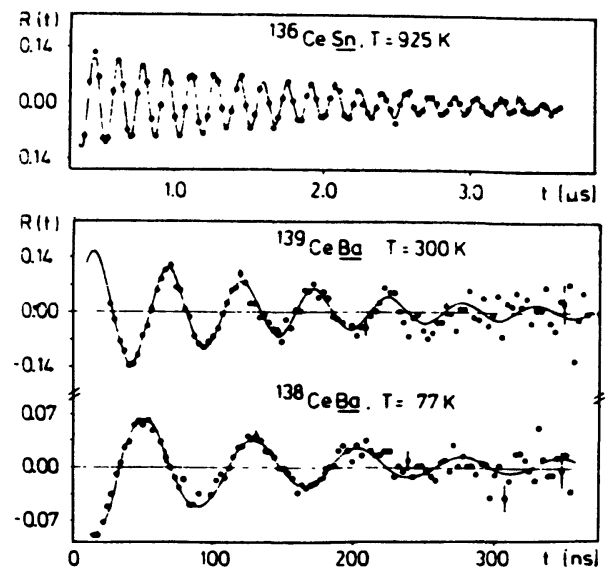

Fig. 11. - (D'après [45b]) Exemples du signal de « rotation de spin " $R(t)$ (expression (91)) de ${ }^{136} \mathrm{CeSn}(552 \mathrm{keV}$; $19,8 \mathrm{kG}),{ }^{139} \mathrm{CeBa}(1607 \mathrm{keV} ; 21,8 \mathrm{kG})$ et ${ }^{138} \overline{\mathrm{CeB}}(430 \mathrm{keV}$; $21,8 \mathrm{kG})$. Les valeurs entre parenthèses correspondent aux énergies des transitions $\gamma$ et aux champs magnétiques utilisés.

[(After [45b]) Examples of the "spin rotation " signal $R(t)$ (expression (91)) of ${ }^{136} \mathrm{CeSn}(552 \mathrm{keV} ; 19,8 \mathrm{kG}),{ }^{139} \mathrm{CeBa}$ $1607 \mathrm{leV} ; 21,8 \mathrm{kG})$ and ${ }^{1 \overline{38}} \mathrm{CeBa}(430 \mathrm{keV} ; 21.8 \mathrm{kG})$. The values in brackets correspond to the $\gamma$ transition energies and to the applied magnetic fields.] 
temps de relaxation électronique $T_{1}$ par une formule analogue à (90), les mesures étant effectuées à haute température et les valeurs de $T_{1}$ de l'ordre de $10^{-12} \mathrm{~s}$ $10^{-13} \mathrm{~s}$.

c) Depuis une dizaine d'années, la dynamique des moments électroniques localisés dans les alliages dilués a été étudiée à l'aide de la résonance magnétique nucléaire [46]. Cette technique est l'analogue de la R.P.E., la puissance de radio-fréquence étant absorbée entre les niveaux Zeeman de l'état fondamental nucléaire. La R.M.N. est donc une mesure directe sur le système nucléaire, et les fréquences résonnantes sont de l'ordre du $\mathrm{MHz}$ pour un champ magnétique appliqué de $10 \mathrm{kG}$.

Dans les alliages dilués contenant des impuretés paramagnétiques, la relaxation du moment nucléaire du noyau de l'impureté ou d'un noyau voisin est dominée par l'interaction hyperfine $\left(A_{\mathrm{hpf}}\right)$ respectivement directe ou transférée par l'intermédiaire des électrons de conduction. Le moment électronique lui-même fluctue sous l'influence du couplage d'échange $\left(J_{\mathrm{ex}}\right)$ avec les électrons de conduction (Fig. 12).

Nous avons aussi représenté sur la figure 12 le couplage direct entre spins nucléaires et électrons de conduction (interaction de contact $A_{\mathrm{c}}^{\prime}$ ) qui conduit à des fréquences de relaxation nucléaires (mécanisme de Korringa) inférieures à $100 \mathrm{~Hz}$ [1] à la température ambiante.

Considérons un doublet de Kramers électronique $(S=1 / 2)$ couplé à un spin nucléaire $I=1 / 2$. Dans les expériences effectuées sous un champ $H_{0}$ de $10 \mathrm{~kg}$ ou plus, on a : $g \mu_{\mathrm{B}} H_{0} \gg A_{\mathrm{hpf}}$, et le système possède un hamiltonien diagonal :

$$
\begin{aligned}
& \mathscr{H}_{0}=-g \mu_{\mathrm{B}} H_{0} S_{\mathrm{z}}+A_{\mathrm{hpf}} I_{\mathrm{z}} S_{\mathrm{z}}-g_{\mathrm{n}} \mu_{\mathrm{n}} H_{0} I_{\mathrm{z}} \\
& \mathscr{H}_{0}=-g \mu_{\mathrm{B}} H_{0} S_{\mathrm{z}}+\omega_{\mathrm{n}} I_{\mathrm{z}}\left(1+\frac{A_{\mathrm{hpf}}}{\omega_{\mathrm{n}}} S_{\mathrm{z}}\right)
\end{aligned}
$$

où $A_{\text {hpf }}$ est la constante hyperfine supposée isotrope, et : $\omega_{\mathrm{n}}=-g_{\mathrm{n}} \mu_{\mathrm{n}} H_{0}$. Le schéma de niveaux est donné sur la figure 13.

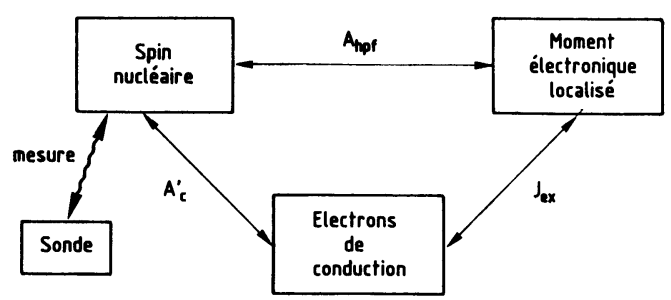

Fig. 12. - Représentation schématique des interactions entre spins nucléaires et spins électroniques dans les alliages dilués (voir texte).

[Schematic representation of the interactions between nuclear spins and electronic spins in dilute alloys (see text).]

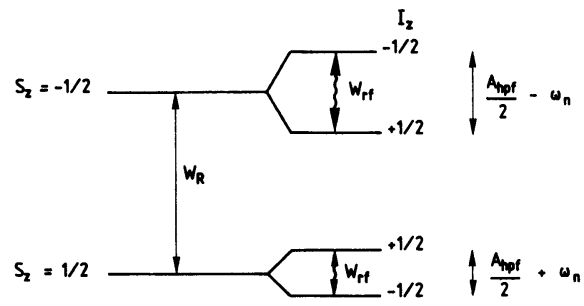

Fig. 13. - Schéma de niveaux électronucléaires pour $S=1 / 2$ et $I=1 / 2$ lorsque $: g \mu_{\mathrm{B}} H_{0} \gg A_{\mathrm{hpf}}$. Transitions R.M.N. : $\$$; transitions de relaxation : $\downarrow$.

[Electronuclear level scheme for $S=1 / 2$ and $I=1 / 2$ when : $g \mu_{\mathrm{B}} H_{0} \gg A_{\mathrm{hf}}$. NMR transitions : $;$ relaxation transitions : $\uparrow$.

Les champs hyperfins étant de l'ordre de $100 \mathrm{kG}$ (pour les ions de transition) ou même $1000 \mathrm{kG}$ (pour les terres rares), on a en général : $A_{\mathrm{hpf}} \gg \omega_{\mathrm{n}}$, et les deux transitions nucléaires ont des énergies $\frac{A_{\mathrm{hpf}}}{2}+\omega_{\mathrm{n}}$ et $\frac{A_{\mathrm{hpf}}}{2}-\omega_{\mathrm{n}}$, séparées par : $2 \omega_{\mathrm{n}}$. Dans les alliages dilués, la fréquence de relaxation électronique $W_{\mathrm{R}}$ de l'impureté est, pour $T>1 \mathrm{~K}$, supérieure à $10^{8} \mathrm{~Hz}$ (cf. relations (19) et $\left(19^{\prime}\right)$ du $\S 2$ ). Donc : $W_{R} \gg 2 \omega_{n}$. Cette condition (cf. $\S 4.3$ ) est celle du « rétrécissement extrême ", c'est-à-dire que la structure du spectre est effacée par la relaxation. En effet, expérimentalement, on observe dans les alliages dilués une seule raie centrée en :

$$
\omega_{0}=\omega_{\mathrm{n}}+A_{\mathrm{hpf}}\left\langle S_{\mathrm{z}}\right\rangle=\omega_{\mathrm{n}}(1+K),
$$

où $K$ est le «Knight-shift » de la raie, donné par :

$$
\begin{aligned}
K & =+\frac{A_{\mathrm{hpf}}}{\omega_{\mathrm{n}}}\left\langle S_{\mathrm{z}}\right\rangle=+\frac{A_{\mathrm{hpf}}}{\omega_{\mathrm{n}}} \frac{\chi_{0} H_{0}}{g \mu_{\mathrm{B}}} \\
& =-\frac{A_{\mathrm{hpf}}}{g_{\mathrm{n}} g \mu_{\mathrm{n}} \mu_{\mathrm{B}}} \chi_{0},
\end{aligned}
$$

$\chi_{0}$ étant la susceptibilité statique du système électronique.

Dans cette limite du rétrécissement extrême, les temps de relaxation nucléaires induits par l'interaction hyperfine : $A_{\mathrm{hpf}}$ I.S sont donnés par les expressions (30) et (54), valables pour une transition unique, où l'on remplace : $\mathbf{H}_{1}(\mathrm{t})$ par : $A_{\mathrm{hpf}} \mathrm{S}(\mathrm{t})$.

Ces temps de relaxation $T_{1 \mathrm{n}}$ et $T_{2 n}$, qui dépendent des fréquences de relaxation électroniques $1 / T_{1 \mathrm{e}}$ et $1 / T_{2 \mathrm{e}}$ (cf. [46] et [12]), sont de l'ordre de $10^{-4} \mathrm{~s}-10^{-5} \mathrm{~s}$, et sont beaucoup plus courts que les temps de relaxation spin-réseau (Korringa ou phonons) des noyaux. Dans la majorité des expériences, ils sont mesurés par la méthode d'analyse temporelle dite des " échos de spin " [47], qui donne accès au temps de relaxation transverse $T_{2 n}$, et qui permet d'éliminer les mécanismes statiques d'élargissement des raies nucléaires 
(distribution d'interactions quadrupolaires par exemple). L'élargissement "dynamique " $1 / T_{2 n}$ dans les alliages dilués est en effet bien inférieur à la largeur statique $\Gamma_{\text {exp }}$. La méthode des " échos de spin ", dans ce cas, remplace avantageusement l'étude de la largeur de raie pour mesurer $T_{2 n}$. On fait subir à l'aimantation nucléaire $M$, dirigée suivant le champ appliqué à l'équilibre thermique (a sur figure 14), une rotation de $90^{\circ}$ qui l'amène suivant $\mathrm{O} x(\mathrm{~b})$, à l'aide d'une impulsion radio-fréquence convenable (si $h_{1} \cos \omega t$ représente le champ de radio-fréquence, la durée $\delta t$ de l'impulsion est telle que : $g_{\mathrm{n}} \mu_{\mathrm{n}} h_{1} \delta t=\pi / 2$ ). L'aimantation se met alors à tourner autour de $H_{0}$ avec une dispersion des fréquences de Larmor $\Gamma_{\mathrm{ex}}$, due aux inhomogénéités microscopiques (c). L'aimantation totale transverse s'annule donc environ en un temps $T_{2}^{*}$, tel que $\Gamma_{\text {ex }} T_{2}^{*} \sim 1$, car la dispersion angulaire sera alors d'environ 1 radian. Au bout d'un temps $\tau$, on applique une impulsion de radio-fréquence à $180^{\circ}$, qui renverse les directions des aimantations $\mathbf{M}_{i}$ des "paquets de spins " (d). On observe alors une " refocalisation " au bout d'un temps $\tau$, c'est-à-dire au bout de $2 \tau$ après l'impulsion à $90^{\circ}$ initiale (e). L'aimantation transverse a décru sous l'effet de la relaxation, et vaut à cet instant : $\mathrm{M} \mathrm{e}^{-2 \tau / T_{2 n}}$. Ce signal, appelé "écho", consiste donc en une émission de radio-fréquence perpendiculairement à $H_{0}$, et décroît en un temps de l'ordre de $T_{2}^{*} \sim 1 / \Gamma_{\mathrm{ex}}$. La mesure de $T_{2 \mathrm{n}}$ par cette méthode exige d'une part que la durée $\delta t$ de l'impulsion à $90^{\circ}$ soit bien inférieure à $T_{2 n}$, et d'autre part que le champ radio-fréquence $h_{1}$ soit tel que : $g_{\mathrm{n}} \mu_{\mathrm{n}} h_{1}>\Gamma_{\mathrm{ex}}$, pour obtenir une rotation de l'ensemble des « paquets de spins " de la raie.

La R.M.N. dans les alliages dilués a été utilisée avec succès dans l'étude de la dynamique des impuretés de transition possédant des propriétés Kondo (CuFe [48], CuMn [49]). La résonance électronique de telles impuretés est impossible à observer à cause de l'effet de "goulot d'étranglement" par les électrons de conduction. Cet effet apparaît pour les ions de transition, car ceux-ci, ayant leur moment orbital " bloqué " par le champ cristallin, possèdent un facteur spectroscopique : $g=2$ égal à celui des électrons de conduction. Le seul alliage Kondo connu d'Ytterbium, $\mathrm{AuYb}$, a été également étudié par R.M.N. [50].

\section{3 EFfacement De LA STRUCTURe DES SPECTRes PAR} LA RELAXATION. - Lorsque le système atomique possède plusieurs transitions spectroscopiques (c'està-dire dont les niveaux peuvent être couplés par l'interaction avec la sonde), le spectre en énergie des probabilités de transition se compose, en l'absence de relaxation, de plusieurs raies (expression (41)).

La levée de dégénérescence peut être due soit à l'interaction hyperfine ("structure hyperfine" des spectres en spectroscopie Mössbauer ou R.P.E.), soit au champ cristallin (structure des spectres de diffusion inélastique de neutrons) ou à une combinaison de ce dernier et de l'énergie Zeeman, lorsque ces deux inter-

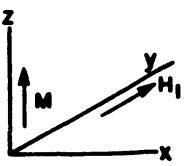

(a)

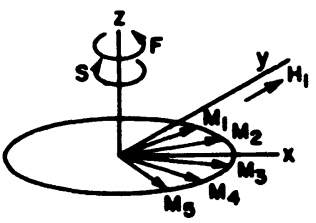

(c)

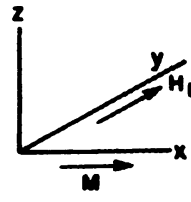

(b)

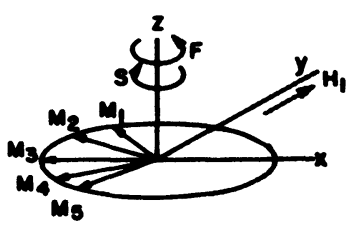

(d)

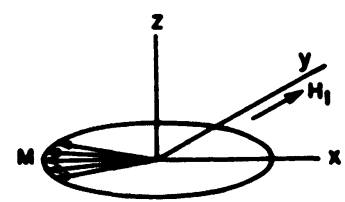

(e)

Fig. 14. - (D'après [47]) Aimantation $M$ des "paquets de spins " pendant une séquence à deux impulsions génératrice d'échos de spin (dans le référentiel tournant) $: a$ et $b$ : Avant et après l'impulsion à $90^{\circ}$. $\mathrm{c}$ et $\mathrm{d}$ : Avant et après l'impulsion à $180^{\circ} . F$ (resp. $S$ ) représente le sens de rotation des paquets de spin les plus rapides $M_{1}$ et $M_{2}$ (resp. les plus lents $M_{4}$ et $M_{5}$ ). e : Refocalisation de tous les paquets de spin à l'instant de l'écho.

[(After [47]) Magnetization $M$ of « spin packets " during a two-pulse sequence which generates the spin echos (in the rotating frame) $: a$ and $b$ : before and after the $90^{\circ}$-pulse. $\mathrm{c}$ and $\mathrm{d}$ : before and after the $180^{\circ}$-pulse. $F$ (resp. $S$ ) represents the rotation direction of the most rapid spin packets $M_{1}$ and $M_{2}$ (resp. of the slowest spin packets $M_{4}$ and $M_{5}$ ). $\mathrm{e}:$ Focusing of all the spin packets at the instant of the echo.]

actions sont du même ordre de grandeur (« structure fine » des spectres de R.P.E. de l'ion $S \mathrm{Gd}^{3+}$ ). En résonance magnétique nucléaire, les spectres possèdent souvent une structure due à l'interaction hyperfine quadrupolaire, qui peut être du même ordre de grandeur que l'énergie Zeeman.

Le problème de l'effet de la relaxation électronique sur le spectre ne peut alors se réduire à un élargissement de chaque raie, sauf dans des circonstances très particulières. Nous avons vu, par exemple, que dans le cas de la spectroscopie Mössbauer, l'effet des transitions induites par la relaxation équivaut à une modulation de la fréquence spectroscopique. Cet effet de modulation a été analysé dans le cas de la R.M.N. d'un proton sautant entre deux (ou plusieurs) environnements chimiques dans lesquels la fréquence de résonance est différente [51, 1], ainsi que dans le cas de la raie de résonance électronique d'un ensemble de moments magnétiques couplés par l'échange [52]. Si $\Delta \omega$ est l'écart entre les deux fréquences de réso- 
nance du proton, ou la largeur qu'aurait la raie R.P.E. en présence des seules interactions dipolaires, on obtient un effacement de la structure (« motional narrowing ") ou un affinement de la raie (" exchange narrowing ") lorsque $: W \sim \Delta \omega$, où $W$ est la fréquence de saut du proton ou la constante d'échange entre spins électroniques.

Dans la limite : $W \gg \Delta \omega$, la résonance a lieu pour une fréquence moyenne $\bar{\omega}$, et la raie est élargie d'une quantité :

$$
\Delta \Gamma \simeq \frac{(\Delta \omega)^{2}}{W}
$$

Ces effets sont décrits en détail à l'aide d'une théorie stochastique de la forme de raie par Kubo [53].

Dans le cas de la relaxation d'une impureté paramagnétique, deux phénomènes peuvent être présents : une modulation des énergies des transitions spectroscopiques, induite par les couplages de relaxation entre des états appartenant à deux transitions différentes, et un effet d'élargissement, induit par les couplages de relaxation entre deux états d'une même transition spectroscopique.

Pour mettre en évidence l'analogie entre le rétrécissement du spectre R.M.N. du proton sautant entre deux fréquences de résonance (illustré par la Fig. 15) et le rétrécissement dû à la relaxation électronique, on peut considérer un système ayant deux transitions spectroscopiques $|1\rangle \leftrightarrow|2\rangle$ et $|3\rangle \leftrightarrow|4\rangle$ (Fig. 16), le hamiltonien de relaxation n'ayant d'éléments de matrice qu'entre les états $|1\rangle$ et $|3\rangle$ d'une part, $|2\rangle$ et $|4\rangle$ d'autre part (un tel système peut simuler la situation rencontrée en spectroscopie Mössbauer). La forme de raie d'absorption s'obtient à partir des équations d'évolution de $\sigma_{12}(t)$ et $\sigma_{34}(t)$.

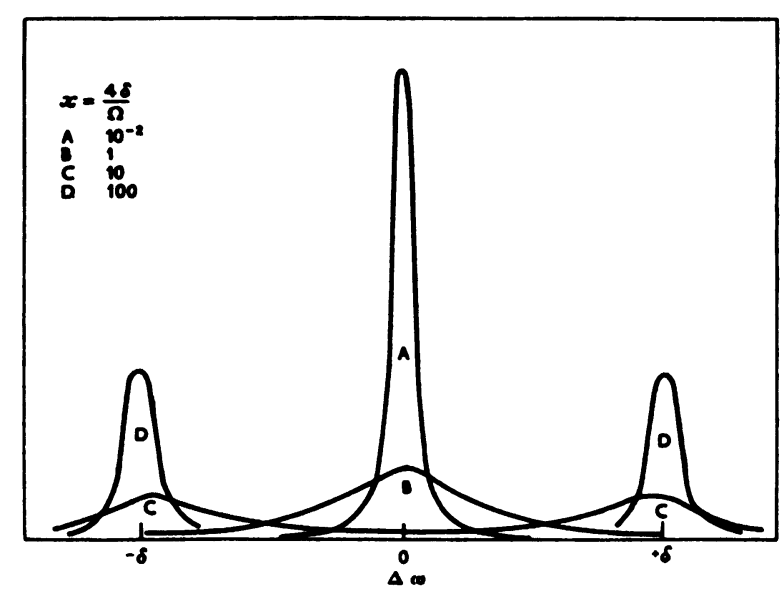

Fig. 15. - (D'après [1]) Forme théorique du spectre d'un système ayant deux fréquences de résonance $\pm \delta$, sautant de l'une à l'autre avec une fréquence moyenne $: 1 / \tau=\Omega$.

[(After [1]) Theoretical lineshape of a system possessing two resonance frequencies $\pm \delta$, jumping from one to the other with an average frequency $: 1 / \tau=\Omega$.]

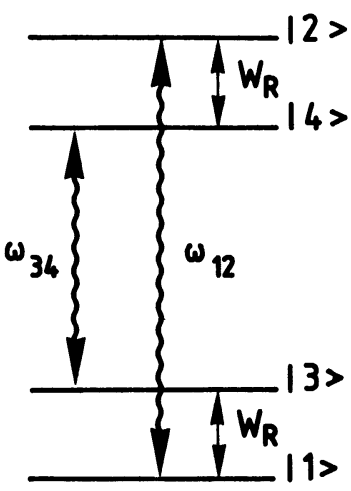

Fig. 16. - Schéma d'énergie fictif illustrant le rétrécissement par le mouvement ; flèches droites : transitions de relaxation; flèches ondulées : transitions spectroscopiques.

[Fictitious energy diagram illustrating the " motional narrowing "; straight arrows : relaxation transitions; waved arrows : spectroscopic transitions.]

Avec les hypothèses simplificatrices de haute température, d'isotropie de la relaxation et de "forces d'oscillateur » identiques pour les deux transitions, on montre sans peine que les équations d'évolution (14) s'écrivent :

$$
\left.\begin{array}{l}
\frac{\mathrm{d} \sigma_{12}}{\mathrm{~d} t}=+i \omega_{12} \sigma_{12}-2 W_{\mathrm{R}} \sigma_{12}+2 W_{\mathrm{R}} \sigma_{34} \\
\frac{\mathrm{d} \sigma_{34}}{\mathrm{~d} t}=+i \omega_{34} \sigma_{34}-2 W_{\mathrm{R}} \sigma_{34}+2 W_{\mathrm{R}} \sigma_{12},
\end{array}\right\}
$$

où :

$$
\begin{aligned}
W_{\mathrm{R}} & =-\frac{1}{2} S_{12,34}=+\frac{1}{2} S_{12,12}=+\frac{1}{2} S_{34,34} \\
& =-\frac{1}{2} S_{34,12} .
\end{aligned}
$$

Ces équations sont formellement identiques aux équations de la référence [51] décrivant l'évolution des deux composantes de la fonction de relaxation, et le calcul de la forme de raie conduit à un résultat identique, dépendant du paramètre : $x=\frac{\omega_{12}-\omega_{34}}{W_{\mathbf{R}}}$ (Fig. 15). Lorsque : $x \gg 1$, c'est-à-dire lorsque la fréquence de relaxation électronique est bien inférieure à l'écart en fréquence entre les raies (relaxation "lente»), le spectre se compose de deux raies Lorentziennes, sans recouvrement, centrées en $\omega_{12}$ et $\omega_{34}$, dont l'élargissement dynamique vaut : $W_{\mathbf{R}}$.

L'accélération de la relaxation élargit progressivement les raies et brouille le spectre, jusqu'à ce que, lorsque : $W_{\mathbf{R}} \sim \omega_{12}-\omega_{34}$, la structure ait disparu. Enfin, en relaxation « rapide» $(x \leqslant 1)$, la raie est 
Lorentzienne, centrée en $\bar{\omega}=\frac{\omega_{12}+\omega_{34}}{2}$, et possède un élargissement dynamique :

$$
\Delta \Gamma_{\mathrm{R}}=\frac{\left(\omega_{12}-\omega_{34}\right)^{2}}{16 W_{\mathrm{R}}}
$$

En fait, on peut distinguer trois situations quant à l'influence de la relaxation électronique sur un spectre à plusieurs raies :

i) "Rétrécissement extrême " lorsque la relaxation devient très rapide : c'est le cas que nous venons de décrire. Il se produit lorsque les niveaux spectroscopiques ne sont pas couplés par l'interaction de relaxation électronique comme en spectrométrie Mössbauer, où l'évolution du spectre est analogue à celle de la figure 15. La région : $W_{\mathbf{R}} \lesssim \omega_{\text {hpf }}$ constitue alors la " fenêtre » où l'on peut mesurer la fréquence de relaxation avec le plus de précision. Le domaine de la relaxation rapide est inexploitable par suite de la largeur «naturelle" finie $\Gamma_{\text {in }}$ de la raie Mössbauer; par contre, les mesures de corrélations angulaires perturbées $\gamma-\gamma$, n'effectuant pas d'analyse en énergie, permettent de mesurer les temps de relaxation très courts, car l'inverse de $\Delta \Gamma_{\mathrm{R}}$ (relation (97)) peut alors être considéré comme le temps de relaxation nucléaire $T_{\text {nucl }}$ :

$$
\Delta \Gamma_{\mathrm{R}} \sim \frac{1}{T_{\text {nucl }}} \sim \omega_{\mathrm{hpf}}^{2} T_{1} \quad(\text { cf. relation }(90)) .
$$

On voit que, lorsque la fréquence de relaxation électronique $1 / T_{1}$ devient très rapide, le noyau " n'a pas le temps de suivre ", et le temps de relaxation nucléaire $T_{\text {nucl }}$ augmente, permettant une mesure aisée par les méthodes d'analyse en temps.

Les expériences de R.M.N. dans les alliages dilués sont en général réalisées dans cette situation de " rétrécissement extrême ": $1 / T_{1} \gg \omega_{\mathrm{n}}$.

ii) Rétrécissement du spectre lorsque : $W_{\mathbf{R}} \sim$ $\omega_{21}-\omega_{34}$, puis élargissement progressif de la raie unique lorsque la relaxation s'accélère : ce phénomène se produit lorsque, outre l'effet de modulation, sont présents des couplages de relaxation entre les niveaux définissant les transitions spectroscopiques. Par exemple, la structure fine du spectre de résonance de $\mathrm{Gd}^{3+}$ dans le palladium (Fig. 17, d'après la Réf. [54]) est effacée par la relaxation due au couplage d'échange avec les électrons de conduction lorsque : $\boldsymbol{W}_{\mathrm{R}} \sim$ $1 \mathrm{GHz}$, c'est-à-dire est du même ordre de grandeur que les écarts de champ cristallin (au second ordre) entre les raies $(\sim 0,1 \mathrm{~K})$. A plus haute température, la raie de résonance s'élargit de la même manière que pour un spin $S=1 / 2$, toute structure ayant disparu.

On retrouve un comportement analogue en spectroscopie de perte ou gain d'énergie des neutrons : la structure de champ cristallin disparait lorsque $W_{\mathbf{R}} \sim$ $\Delta_{\mathrm{cc}}\left(\Delta_{\mathrm{cc}}\right.$ : écart entre deux doublets de champ cristallin) puis le spectre se fond en une raie quasi-élastique, dont

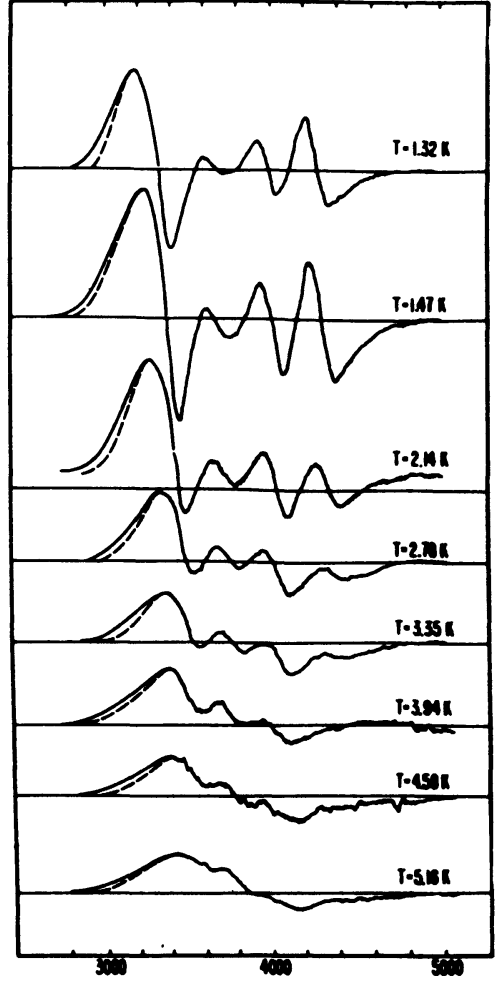

Fig. 17. - (D'après [54]) Spectres R.P.E. dans $\mathbf{P d}: \mathbf{G d}^{3+}$ $(300 \mathrm{ppm})$ à différentes températures en fonction du champ magnétique appliqué suivant la direction [001].

[(After [54]) E.P.R. spectra of $\mathbf{P d}: \mathrm{Gd}^{3+}(300 \mathrm{ppm})$ at different temperatures as a function of the applied magnetic field along [001].]

l'élargissement dynamique vaut $W_{R}$ et croît lorsque la température augmente [33c].

iii) Enfin, en l'absence d'effet de modulation, les différentes raies du spectre évoluent indépendamment : le cas de la résonance électronique en présence d'interaction hyperfine, lorsque : $A_{\mathrm{hpf}} \ll \omega_{0}=g \mu_{\mathrm{B}} H_{0}$, illustre cet effet (Fig. 18). La relaxation électronique conserve le nombre quantique nucléaire $m_{\mathrm{I}}$ et n'induit de transitions qu'entre les niveaux spectroscopiques (énergies $\omega_{0}-A$ et $\omega_{0}+A$ ). Chaque raie est une lorentzienne de largeur dynamique $1 / T_{2}$, et le spectre

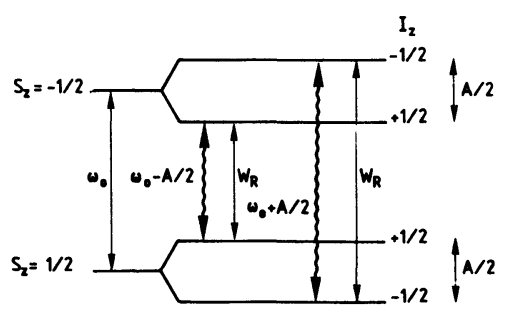

Fig. 18. - Schéma de niveaux hyperfins en champ fort $\left(g \mu_{\mathrm{B}} H_{0} \gg A_{\mathrm{hpf}}\right)$ pour $S=1 / 2$ et $I=1 / 2$; transitions R.P.E. : $\uparrow$; transitions de relaxation : $\uparrow$.

[Hyperfine level scheme in the strong field regime $\left(g \mu_{\mathrm{B}} H_{0} \gg\right.$ $\left.A_{\mathrm{hf}}\right)$ for $S=1 / 2$ and $I=1 / 2$; E.P.R. transitions : $\$$; relaxation transitions : $\uparrow$.] 
s'élargit sans se brouiller lorsque $1 / T_{2}$ augmente. Les études par R.P.E. des alliages dilués de terre rare $\mathrm{AgEr}^{3+}$ [55], et $\mathrm{AuEr}^{3+}$ et $\mathrm{AuYb}^{3+}$ [56] ont mis cet effet en évidence, tout phénomène de "bottleneck » étant absent dans ces alliages $\left(g_{\mathrm{TR}} \neq 2\right)$. Par contre, lorsque l'impureté est un ion de transition ou un ion $\mathrm{S}$ comme $\mathrm{Gd}^{3+}\left(g_{\text {ion }}=2\right)$, la résonance est profondément affectée par le "goulot d'étranglement ", qui se traduit dans les métaux par une précession conjointe des aimantations ioniques et des électrons de conduction. On peut alors montrer que dans ce cas, le spectre hyperfin peut être "effacé " par l'échange avec les électrons itinérants, lorsque : $W_{\mathrm{R}} \gtrsim A_{\mathrm{hpf}}[57,58]$.

Dans les situations i) et ii) que nous venons de décrire, l'effet complexe de la relaxation sur le spectre en énergie nécessite le calcul de la fonction de relaxation :

$$
G(t)=\left\langle M^{+}(t) M\right\rangle .
$$

Dans le cadre d'un traitement en perturbation de la relaxation, il existe deux méthodes principales : l'une à partir de l'évolution de la matrice densité, que nous avons utilisée dans cet article, et une méthode dite de la "résolvante", fondée sur le formalisme des projecteurs de Mori [64]. L'application de ces méthodes au calcul de la forme de raie en spectroscopie Mössbauer est décrite en détail dans la référence [42]. La susceptibilité magnétique $\chi(\omega)$ d'une impureté paramagnétique dans un métal en présence de relaxation a été calculée de diverses manières : par la méthode des projecteurs de Mori dans le cas de la R.P.E. [60] et de la diffusion inélastique des neutrons [62], par une méthode diagrammatique [61] et à partir d'équations de Bloch « microscopiques » [59], toujours en R.P.E. La moyenne sur le bain apparaissant dans la fonction de relaxation $G(t)$ peut également être effectuée par des méthodes probabilistes, ou "stochastiques". Celles-ci sont particulièrement bien adaptées lorsque la relaxation se réduit à un effet de modulation des fréquences de transition. Une bonne revue des calculs stochastiques de forme du signal observé par les techniques " hyperfines" est parue récemment [63].

\section{Dépendances thermiques des fréquences de relaxa- tion.}

Nous allons examiner dans ce paragraphe les mécanismes de relaxation d'une impureté paramagnétique par l'interaction avec les vibrations du réseau et les électrons de conduction. Nous avons vu que, dans ces deux cas, un traitement perturbatif de la relaxation est valable. De plus, il est possible de calculer quantiquement les densités spectrales associées. La dépendance thermique des éléments de la matrice de relaxation $S_{a a^{\prime}, b b^{\prime}}(T)$ est contenue toute entière dans ces densités spectrales $J(\omega, T)$, et nous allons voir que les modèles utilisés pour calculer $J(\omega, T)$ reproduisent à une très bonne approximation les dépendances thermiques observées expérimentalement.
5.1 Relaxation par les phonons. - La partie dynamique de l'interaction de champ cristallin, associée aux vibrations thermiques du réseau ionique, crée sur un site donné des champs électriques fluctuants qui perturbent le mouvement orbital des électrons atomiques. Si $V(\mathbf{r})$ est l'énergie potentielle électrostatique au point $r$ proche d'un site atomique, en supposant toutes les charges du réseau fixes, l'énergie $V^{\prime}(\mathbf{r})$ obtenue après un petit déplacement relatif $\left(u_{i}, v_{i}, w_{i}\right)$ de l'ion $\underline{i}$ peut être développée en puissances des déplacements :

$$
\begin{aligned}
V^{\prime}(\mathbf{r})=V(\mathbf{r})+\sum_{i}\left(u_{i} \frac{\partial V}{\partial x}\right. & \left.+v_{i} \frac{\partial V}{\partial y}+w_{i} \frac{\partial V}{\partial z}\right)+ \\
& +\frac{1}{2} \sum_{i} u_{i}^{2} \frac{\partial^{2} V}{\partial x^{2}}+\cdots
\end{aligned}
$$

La partie statique $V(\mathbf{r})$ détermine les niveaux de champ cristallin de l'ion, et la partie dynamique, où les déplacements peuvent être considérés comme des fonctions aléatoires du temps $u_{i}(t), v_{i}(t)$ et $w_{i}(t)$, induit des transitions entre les fonctions d'onde orbitales.

Cet hamiltonien peut être transformé en utilisant la quantification des vibrations du réseau en phonons; on exprime alors les déplacements $u_{i}, v_{i}, w_{i}$ en fonction des opérateurs de création $a_{k}^{+}$et d'annihilation $a_{k}$ d'un phonon de vecteur d'onde $\mathbf{k}[65,66]$. Les fonctions $\partial V / \partial x, \partial V / \partial y$, etc... s'expriment en fonction des composantes de l'opérateur moment orbital $\mathbf{L}$ total de l'ion, ou du moment total $\mathbf{J}$ pour une terre rare (théorème de Wigner-Eckart). L'interaction dynamique orbite-réseau prend alors la forme [65] :

$$
\begin{aligned}
\mathscr{H}_{\mathrm{or}}=\sum_{\mathbf{k}} & |\mathbf{k}| \sqrt{\frac{\hbar}{2 M \omega_{k}}}\left(a_{k}+a_{k}^{+}\right) U_{k}(\mathbf{J})+ \\
& +\sum_{k, \mathbf{k}^{\prime}}|\mathbf{k}|\left|\mathbf{k}^{\prime}\right| \sqrt{\frac{\hbar}{2 M \omega_{k}}} \sqrt{\frac{\hbar}{2 M \omega_{k^{\prime}}}} \\
& \times\left(a_{k}+a_{k}^{+}\right)\left(a_{k^{\prime}}+a_{k^{\prime}}^{+}\right) U_{k k^{\prime}}(\mathbf{J})+\cdots
\end{aligned}
$$

Dans cette formule, $\omega_{k}$ est l'énergie de la branche de phonons concernée, $M$ la masse de l'atome, $U_{k}(\mathbf{J})$ et $U_{k k^{\prime}}(\mathbf{J})$ des " opérateurs-équivalent " de champ cristallin ayant la dimension d'une énergie (nous avons particularisé au cas des terres rares). Comme les opérateurs du champ cristallin statique, $U_{k}(J)$ et $U_{k k^{\prime}}(J)$ n'ont pas d'éléments de matrice entre deux états conjugués d'un doublet de Kramers. En outre, dans le cas d'un ion $S(L=0), U_{k}(J)$ est une quantité du second ordre, et l'on doit s'attendre à ce que le couplage spin-phonons pour les ions $\mathrm{S}\left(\mathrm{Mn}^{2+}, \mathrm{Fe}^{3+}\right.$, $\mathrm{Eu}^{2+}, \mathrm{Gd}^{3+}$ ) soit beaucoup plus faible que pour les ions possédant un moment orbital. Ceci se traduit par le fait que les fréquences de relaxation spin-phonons des ions $S$ sont relativement « lentes ", même à haute température (cf. Fig. 27, $\S 5.1$.iv).

Nous allons ici indiquer les grandes lignes du calcul 
des dépendances thermiques des fréquences de relaxation entre deux états électroniques $|a\rangle$ et $|b\rangle$ séparés par une énergie $\hbar \omega_{a b}$. Nous nous limiterons aux processus les plus fréquemment rencontrés.

Schématiquement, nous considérerons les phonons acoustiques dans l'approximation de Debye $(\omega=v k$, où $v$ est la vitesse du son dans le solide), et une branche de phonons optiques dans l'approximation d'Einstein. La densité d'états $g(\omega)$ des branches acoustiques et optiques de phonons est représentée schématiquement sur la figure 19.

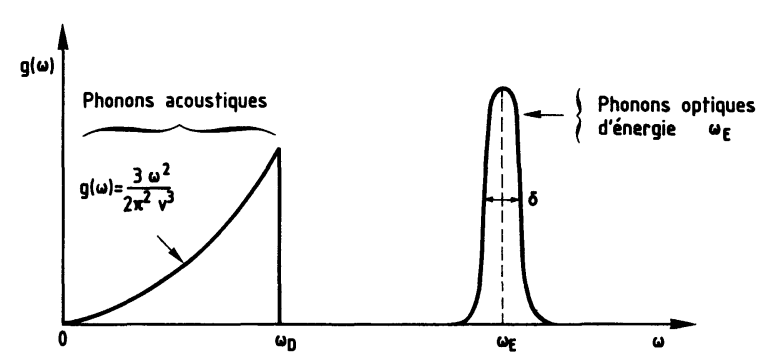

Fig. 19. - Densité d'états schématique des phonons (par unité de volume). $\omega_{\mathrm{D}}$ est la fréquence de coupure des phonons acoustiques (fréquence de Debye, reliée à la température de Debye $\theta_{\mathrm{D}}$ par : $\left.\hbar \omega_{\mathrm{D}}=k_{\mathrm{B}} \theta_{\mathrm{D}}\right) ; \omega_{\mathrm{E}}$ est l'énergie de la branche optique, supposée avoir une largeur $: \delta \ll \omega_{\mathrm{E}}$.

[Schematic phonon density of states (per unit volume). $\omega_{\mathrm{D}}$ is the acoustic phonon cut-off frequency (Debye frequency, related to the Debye temperature $\theta_{\mathrm{D}}$ by : $\left.\hbar \omega_{\mathrm{D}}=k_{\mathrm{B}} \theta_{\mathrm{D}}\right)$; $\omega_{\mathrm{E}}$ is the energy of the optical branch, supposed to have a width : $\left.\delta \ll \omega_{\mathrm{E}} \cdot\right]$

Dans l'hamiltonien de couplage $\mathscr{H}_{\text {or }}$, donné par l'expression (99), les termes en $a_{k}$ (resp. $a_{k}^{+}$) décrivent un processus accompagné d'absorption (resp. d'émission) d'un phonon de vecteur d'onde $\mathbf{k}$, les termes en $a_{k} a_{k}^{+}$, des processus à deux phonons. On néglige les processus à 3 phonons dans les calculs de perturbation. Nous considérons d'abord les processus mettant en jeu les branches acoustiques (i, ii, iii), puis les phonons optiques (iv).

i) Les processus directs à un phonon (branche acoustique).

Le terme en $a_{k} U(\mathbf{J})$ de $\mathscr{H}_{\text {or }}$ induit une transition entre les deux états $|a\rangle$ et $|b\rangle$ du doublet, accompagnée d'absorption d'un phonon d'énergie $\hbar \omega_{a b}$. Pour que l'énergie soit conservée, seul le terme en $k_{0}=\omega_{a b} / v$ contribue à ce processus, avec un poids : $g\left(\omega_{a b}\right)$. La probabilité de ce processus s'écrit ( $\mathrm{V}$ est le volume de l'échantillon) :

$$
\begin{array}{r}
W_{a \rightarrow b}=\frac{2 \pi}{\hbar^{2}} k_{0}^{2} \frac{\hbar V}{2 M \omega_{a b}} \mid\left\langle n_{k_{0}}, b\left|a_{k} U_{k_{0}}(\mathbf{J})\right| n_{k_{0}}+\right. \\
+1, a\rangle\left.\right|^{2} g\left(\omega_{a b}\right) \\
W_{a \rightarrow b}=\frac{2 \pi}{\hbar} \frac{k_{0} V}{2 M v} n\left(\omega_{a b}, T\right)\left|\left\langle b\left|U_{k_{0}}(\mathbf{J})\right| a\right\rangle\right|^{2} \times \\
\times g\left(\omega_{a b}\right),(100)
\end{array}
$$

où :

$$
n\left(\omega_{a b}, T\right)=\frac{1}{\mathrm{e}^{h \omega_{a b} / k_{\mathbf{B}} T}-1}
$$

et

$$
g(\omega)=\left\{\begin{array}{ccc}
\frac{3 \omega^{2}}{2 \pi^{2} v^{3}} & \text { si } & \omega<\omega_{\mathrm{D}} \\
0 & \text { si } & \omega>\omega_{\mathrm{D}}
\end{array}\right.
$$

La probabilité d'absorption (par atome) d'un phonon dans un tel processus direct s'écrit alors :

$$
W_{a \rightarrow b}^{\mathrm{d}}=\frac{2 \pi}{\hbar^{2}}\left|\left\langle b\left|U_{k_{0}}(\mathrm{~J})\right| a\right\rangle\right|^{2} J_{a}^{\mathrm{d}}\left(\omega_{a b}\right)
$$

Dans cette expression, $J_{a}^{\mathrm{d}}\left(\omega_{a b}\right)$ est la densité spectrale des phonons acoustiques associée au processus direct d'absorption :

$\begin{cases}J_{a}^{\mathrm{d}}(\omega)=\frac{3 \hbar \omega^{3}}{4 \pi^{2} \rho v^{5}} \frac{1}{\mathrm{e}^{h \omega / k_{\mathrm{B}} T}-1} & \text { pour } \omega<\omega_{\mathrm{D}}, \\ J_{a}^{\mathrm{d}}(\omega)=0 & \text { pour } \omega>\omega_{\mathrm{D}},\end{cases}$

où, $\rho$ est la masse volumique du solide supposé formé de $N$ atomes identiques : $\rho=N M / V$.

On obtiendrait un résultat analogue pour un processus direct mettant en jeu l'absorption d'un photon, avec la différence que la densité d'états $g(\omega)$ des photons s'écrit : $g(\omega)=2 \omega^{2} / 2 \pi^{2} c^{3}$, c'est-à-dire qu'elle est $:(v / c)^{3} \simeq 10^{-18}$ fois plus faible que la densité d'états de phonons. On a représenté schématiquement sur la figure 20 les densités spectrales associées au processus direct d'absorption d'un phonon et d'un photon. A cause du facteur $(v / c)^{3}$, les transitions accompagnées d'absorption de photons ont des probabilités négligeables pour des énergies $\hbar \omega_{a b}$ faibles.

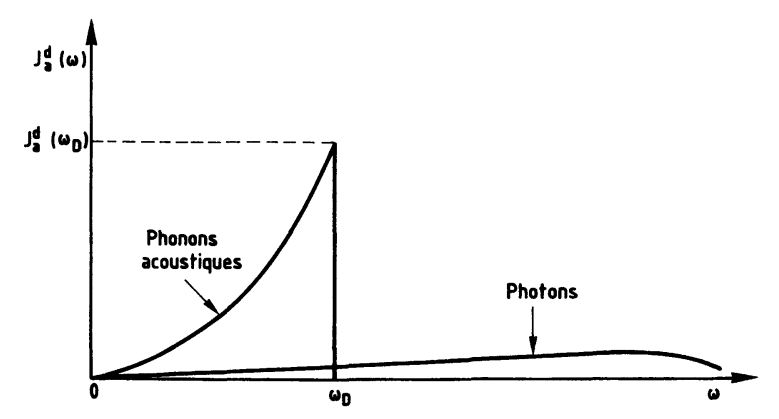

Fig. 20. - Densité spectrale associée au processus direct d'absorption pour les phonons acoustiques et les photons (voir texte).

[Spectral density associated with the direct absorption process for acoustic phonons and photons (see text).]

Par contre, pour des transitions plus énergétiques, comme celles séparant deux multiplets électroniques $\left(\omega_{a b} \simeq\right.$ quelque $\left.1000 \mathrm{~K}\right)$, l'émission spontanée d'un 
photon conduit à basse température à des probabilités de transition " radiative " du même ordre de grandeur que celles des transitions "non-radiatives " mettant en jeu plusieurs phonons optiques. Ces phénomènes de relaxation "intermultiplets " peuvent être étudiés par spectroscopie optique [35].

Il est clair sur la figure 20 que la largeur de la densité spectrale $J^{\mathrm{d}}(\omega)$ est de l'ordre de $\omega_{\mathrm{D}}$, et qu'il en sera de même pour les densités spectrales associées à d'autres processus de relaxation mettant en jeu les phonons acoustiques. Ceci justifie l'application du traitement de perturbation à la relaxation spin-phonon lorsque les fréquences de relaxation sont inférieures à : $1 / \tau_{\mathrm{c}} \sim \omega_{\mathrm{D}} \sim 10^{12}-10^{13} \mathrm{~Hz}$.

La probabilité d'émission d'un phonon entre $|a\rangle$ et $|b\rangle, W_{b \rightarrow a}^{\mathrm{d}}$, se déduit de $W_{a \rightarrow b}^{\mathrm{d}}$ à l'aide du principe de l'équilibre microscopique :

$$
W_{b \rightarrow a}^{\mathrm{d}}=\frac{2 \pi}{\hbar^{2}}\left|\left\langle a\left|U_{k_{0}}(\mathbf{J})\right| b\right\rangle\right|^{2} J_{e}^{\mathrm{d}}\left(\omega_{a b}\right)
$$

où :

$$
J_{e}^{\mathrm{d}}\left(\omega_{a b}\right)=\mathrm{e}^{n \omega_{a b} / k_{\mathrm{B}} T} J_{a}^{\mathrm{d}}\left(\omega_{a b}\right)
$$

L'expression du temps de relaxation $T_{1}$, mesuré par exemple en R.P.E. dans une expérience de saturation par impulsion, est :

$$
\frac{1}{T_{1}^{\mathrm{d}}}=W_{a \rightarrow b}^{\mathrm{d}}+W_{b \rightarrow a}^{\mathrm{d}}(\operatorname{expression}(74)) .
$$

Donc :

$$
\frac{1}{T_{1}^{\mathrm{d}}}=\frac{1}{\hbar}\left|\left\langle a\left|U_{k_{0}}(\mathbf{J})\right| b\right\rangle\right|^{2} \frac{3 \omega_{a b}^{3}}{2 \pi \rho v^{5}} \operatorname{coth} \frac{\hbar \omega_{a b}}{2 k_{\mathrm{B}} T} .
$$

Cette formule est valable pour des états $|a\rangle$ et $|b\rangle$ qui ne sont pas conjugués de Kramers ; si, au contraire, $|a\rangle$ et $|b\rangle$ forment un doublet de Kramers dégénéré (en l'absence de champ magnétique) : $\left\langle a\left|U_{k_{0}}(\mathbf{J})\right| b\right\rangle$ $=0$, et les processus directs sont inexistants.

En présence de champ magnétique $H_{0}$, les états $|a\rangle$ et $|b\rangle$ sont mélangés avec les états $\left|a^{\prime}\right\rangle$ et $\left|b^{\prime}\right\rangle$ du premier doublet excité de champ cristallin, situé à une énergie $\Delta$. Le doublet fondamental devient : $\left(\left|a_{1}\right\rangle,\left|b_{1}\right\rangle\right)$ tels que :

$$
\left\{\begin{array}{l}
\left|a_{1}\right\rangle=|a\rangle+\frac{g \mu_{\mathrm{B}} H_{0}}{\Delta}\left(\alpha\left|a^{\prime}\right\rangle+\beta\left|b^{\prime}\right\rangle\right) \\
\left|b_{1}\right\rangle=|b\rangle+\frac{g \mu_{\mathrm{B}} H_{0}}{\Delta}\left(\alpha^{\prime}\left|a^{\prime}\right\rangle+\beta^{\prime}\left|b^{\prime}\right\rangle\right) .
\end{array}\right.
$$

Donc :

$$
\begin{aligned}
\left\langle a_{1}\left|U_{k_{0}}(\mathbf{J})\right| b_{1}\right\rangle \simeq \frac{g \mu_{\mathrm{B}} H_{0}}{\Delta}\left[\alpha^{\prime}\left\langle a\left|U_{k_{0}}(\mathbf{J})\right| a^{\prime}\right\rangle+\right. \\
+\beta^{\prime}\left\langle a\left|U_{k_{0}}(\mathbf{J})\right| b^{\prime}\right\rangle+\alpha\left\langle a^{\prime}\left|U_{k_{0}}(\mathbf{J})\right| b\right\rangle \\
\left.+\beta\left\langle b^{\prime}\left|U_{k_{0}}(\mathbf{J})\right| b\right\rangle\right],
\end{aligned}
$$

ou bien :

$$
\left\langle a_{1}\left|U_{k_{0}}(\mathbf{J})\right| b_{1}\right\rangle=\frac{\hbar \omega_{a b}}{\Delta} V_{k_{0}}(\mathbf{J}),
$$

en négligeant les termes du second ordre en $\left(\hbar \omega_{a b} / \Delta\right)^{2}$.

On obtient alors pour le temps $T_{1}$ associé aux processus directs entre les deux états d'un doublet de Kramers en présence de champ magnétique :

$$
\frac{1}{T_{1}^{\mathrm{d}}}=\left|V_{k_{0}}(\mathbf{J})\right|^{2} \frac{3 \hbar \omega_{a b}^{5}}{2 \pi \rho v^{5} \Delta^{2}} \operatorname{coth} \frac{\hbar \omega_{a b}}{2 k_{\mathrm{B}} T}
$$

ii) Les processus «Raman" à deux phonons (branche acoustique). Ils proviennent des termes en $a_{k_{1}} a_{k_{2}}^{+} U_{k_{1} k_{2}}(J)$ au premier ordre de perturbation en $\mathscr{H}_{\text {or }}$ (processus " réels ») et des termes croisés en $a_{k_{1}} U_{k_{1}}(\mathbf{J}) a_{k_{2}}^{+} U_{k_{2}}(\mathbf{J})$ obtenus au deuxième ordre de perturbation en $\mathscr{H}_{\text {or }}$ (processus « virtuels ").

Les processus "réels " sont inexistants pour les doublets de Kramers, en l'absence de champ magnétique, car, si $|a\rangle$ et $|b\rangle$ sont conjugués de Kramers : $\left\langle a\left|U_{k_{1} k_{2}}(J)\right| b\right\rangle=0$. Pour les ions non de Kramers, ces processus du $1^{\mathrm{er}}$ ordre sont plus importants que les processus "virtuels " du $2^{e}$ ordre. On peut donc dire que la dépendance thermique des processus Raman vient principalement des processus " réels " pour les ions non de Kramers et des processus « virtuels » pour les ions de Kramers.

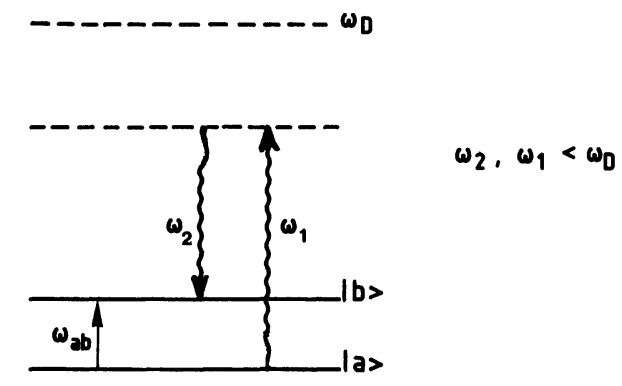

Fig. 21. - Processus Raman "réel " d'absorption à deux phonons acoustiques entre les niveaux ioniques $|a\rangle$ et $|b\rangle$.

[«Real » absorption Raman process with two acoustic phonons between the ionic levels $|a\rangle$ and $|b\rangle$.]

a) Un processus « réel » d'absorption est représenté sur la figure 21. La transition ionique de $|a\rangle$ vers $|b\rangle$ est accompagnée de l'absorption d'un phonon d'énergie $\hbar \omega_{1}$ et de l'émission d'un phonon d'énergie $\hbar \omega_{2}$. La conservation de l'énergie est satisfaite dans ce cas si : $\omega_{a b}=\omega_{2}-\omega_{1}$. La probabilité de transition entre $|a\rangle$ et $\left|b_{2}\right\rangle$ vaut :

$$
\begin{aligned}
& W_{a \rightarrow b}^{\mathrm{R}}=\frac{2 \pi}{\hbar^{2}} \sum_{k_{1}, k_{2}} \frac{\hbar^{2}}{4 M^{2} \omega_{1} \omega_{2}} k_{1}^{2} k_{2}^{2} \times \\
& \times\left|\left\langle b, n_{k_{1}}, n_{k_{2}}+1\left|a_{k_{1}} a_{k_{2}}^{+} U_{k_{1} k_{2}}\right| a, n_{k_{1}}+1, n_{k_{2}}\right\rangle\right|^{2} \\
& \times \delta\left(\omega_{2}-\omega_{1}-\omega_{a b}\right) .
\end{aligned}
$$


Les $n_{k_{i}}$ sont les nombres d'occupation des états $\boldsymbol{k}_{\boldsymbol{i}}$ de phonons.

En s'aidant de la représentation continue des phonons acoustiques, caractérisés par une densité d'états par unité de volume : $g(\omega)=3 \omega^{2} / 2 \pi^{2} v^{3}$, on obtient :

$$
\begin{aligned}
& W_{a \rightarrow b}^{\mathrm{R}}=\frac{2 \pi V^{2}}{4 M^{2}} \int_{0}^{\omega_{\mathrm{D}}} \mathrm{d} \omega_{1} \mathrm{~d} \omega_{2} g\left(\omega_{1}\right) g\left(\omega_{2}\right) \times \\
& \times \frac{\omega_{1} \omega_{2}}{v^{4}} n\left(\omega_{1}, T\right)\left[n\left(\omega_{2}, T\right)+1\right]\left|\left\langle b\left|U_{k_{1} k_{2}}\right| a\right\rangle\right|^{2} \\
& \times \delta\left(\omega_{a b}-\omega_{2}+\omega_{1}\right) .
\end{aligned}
$$

Dans l'approximation où l'on peut considérer que : $\omega_{a b} \ll \omega_{1}, \omega_{2}$, et lorsque : $\hbar \omega_{a b} \ll k_{\mathrm{B}} T$, on a, en posant : $\omega=\omega_{1}=\omega_{2}$ :

$$
\begin{aligned}
& W_{a \rightarrow b}^{\mathrm{R}}=\frac{9}{8 \pi^{3} \rho^{2} v^{10}} \times \\
& \quad \times\left\{\int_{0}^{\omega_{\mathrm{D}}} \mathrm{d} \omega \omega^{6} \frac{\mathrm{e}^{n \omega / k_{B} T}}{\left(\mathrm{e}^{\hbar \omega / k_{B} T}-1\right)^{2}}\right\}\left|\left\langle b\left|U_{2}\right| a\right\rangle\right|^{2},
\end{aligned}
$$

où l'on a supposé que l'élément de matrice $\left\langle b\left|U_{k_{1} k_{2}}\right| a\right\rangle$ est indépendant de $k_{1}$ et $k_{2}$ et vaut : $\left\langle b\left|U_{2}\right| a\right\rangle$.

Donc :

$W_{a \rightarrow b}^{\mathrm{R}}=\frac{9\left|\left\langle b\left|U_{2}\right| a\right\rangle\right|^{2}}{8 \pi^{3} \rho^{2} v^{10}}\left(\frac{k_{\mathrm{B}} T}{\hbar}\right)^{7} I^{6}\left(\frac{\theta_{\mathrm{D}}}{T}\right)$,

où : $\theta_{\mathrm{D}}=\frac{\hbar \omega_{\mathrm{D}}}{k_{\mathrm{B}}}$.

La fonction $I^{6}(x)$ est définie par :

$$
I^{6}(x)=\int_{0}^{x} \frac{y^{6} \mathrm{e}^{-y}}{\left(1-\mathrm{e}^{-y}\right)^{2}} \mathrm{~d} y,
$$

et à température suffisamment basse $\left(\theta_{\mathrm{D}} / T>10\right)$, $I^{6}\left(\theta_{\mathrm{D}} / T\right)$ peut être remplacé par : $I^{6}(\infty)=6$ !.

Le temps de relaxation $T_{1}$, associé aux processus Raman " réels ", pour un ion non de Kramers vaut alors, dans le domaine de température : $\hbar \omega_{a b} \ll$ $k_{\mathrm{B}} T<k_{\mathrm{B}} \theta_{\mathrm{D}} / 10$ :

$$
\begin{aligned}
\frac{1}{T_{1}^{\mathrm{R}}} & =W_{a \rightarrow b}^{\mathrm{R}}+W_{b \rightarrow a}^{\mathrm{R}} \simeq 2 W^{\mathrm{R}} \\
& =\frac{9\left|\left\langle b\left|U_{2}\right| a\right\rangle\right|^{2}}{4 \pi^{3} \rho^{2} v^{10}} 6 !\left(\frac{k_{\mathrm{B}} T}{\hbar}\right)^{7} .
\end{aligned}
$$

Signalons que lorsque l'approximation « haute température » n'est pas vérifiée $\left(\hbar \omega_{a b} \lesssim k_{\mathrm{B}} T\right)$, la dépendance thermique de $1 / T_{1}^{R}$ consiste en un polynôme en $k_{\mathrm{B}} T / \hbar$ comprenant des' termes de degré $7,6,5$ et $4[67]$. b) Les processus « virtuels » font intervenir un état de champ cristallin excité $|c\rangle$. Les probabilités de transition entre $|a\rangle$ et $|b\rangle$ contiennent des éléments de matrice du type :

$$
\begin{aligned}
\sum_{c}\left\langle b, n_{k_{1}}, n_{k_{2}}\right| a_{k_{2}}^{+} & U_{k_{2}}\left|c, n_{k_{1}}, n_{k_{2}}-1\right\rangle \times \\
& \times\left\langle c, n_{k_{1}}, n_{k_{2}}-1\left|\frac{a_{k_{1}} U_{k_{1}}}{\hbar\left(\omega_{1}-\omega_{a c}\right)}\right|\right. \\
& \left.\times a, n_{k_{1}}+1, n_{k_{2}}-1\right\rangle
\end{aligned}
$$

Ce processus d'absorption est schématisé sur la figure 22. La conservation de l'énergie dans un tel processus implique : $\omega_{a b}=\omega_{2}-\omega_{1}$.

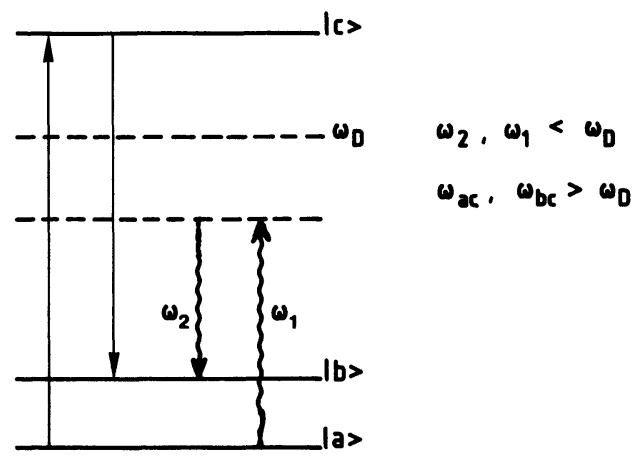

Fig. 22. - Processus Raman « virtuel » d'absorption à deux phonons acoustiques entre les niveaux ioniques $|a\rangle$ et $|b\rangle$.

["Virtual" absorption Raman process with two acoustic phonons between the ionic levels $|a\rangle$ and $|b\rangle$.]

Pour que le traitement au $2^{\mathrm{e}}$ ordre en perturbation converge, il faut que les dénominateurs du type $\omega_{1}-\omega_{a c}$ ne s'annulent pas, c'est-à-dire que le niveau excité de champ cristallin $|c\rangle$ soit tel que : $\omega_{a c}>\omega_{\mathrm{D}}$. Dans le cas contraire, s'il existe un niveau de champ cristallin proche du fondamental, il faut effectuer le calcul de perturbation au $1^{\mathrm{er}}$ ordre à partir des 3 états $|a\rangle,|b\rangle$ et $|c\rangle$ (processus « Orbach », cf. iii).

Lorsqu'on peut considérer que : $\omega_{1} \ll \omega_{a c}$ alors :

$$
\frac{1}{\omega_{1}-\omega_{a c}} \simeq-\frac{1}{\omega_{a c}}\left[1+\frac{\omega_{1}}{\omega_{a c}}+\cdots\right] \text {. }
$$

Pour un ion non de Kramers, on ne considère que le premier terme du développement, et on obtient alors, en parfaite analogie avec le calcul des processus « réels ", un terme en $\left(k_{\mathrm{B}} T / \hbar\right)^{7}$.

Par contre, pour un doublet de Kramers :

$$
\sum_{c} \frac{\left\langle b\left|U_{k_{2}}\right| c\right\rangle\left\langle c\left|U_{k_{1}}\right| a\right\rangle}{\omega_{a c}}=0,
$$

et on doit considérer le $2^{\mathrm{e}}$ terme, qui ne s'annule pas, car les états mixtes $\left|b, n_{k_{1}}, n_{k_{2}}\right\rangle$ et $\mid a, n_{k_{1}}+1, n_{k_{2}}-$ 1 ) ne sont pas conjugués de Kramers. On obtient 
alors, par exemple pour un doublet dégénéré, sans champ magnétique $\left(\omega_{a b}=0\right)$ :

$$
\begin{aligned}
W_{a \rightarrow b}^{\mathrm{R}}=\frac{9}{8 \pi^{3} \hbar^{2} \rho^{2} v^{10}} \times \\
\times \frac{\mid\left\langle\left. b|U| c\right|^{2}|\langle c|U| a\rangle|^{2}\right.}{\omega_{a c}^{4}} \\
\times\left\{\int_{0}^{\omega_{\mathrm{D}}} \mathrm{d} \omega \omega^{8} \frac{\mathrm{e}^{\mathrm{n} \omega / k_{\mathrm{B}} T}}{\left(\mathrm{e}^{\mathrm{n} / k_{\mathrm{B}} T}-1\right)^{2}}\right\},
\end{aligned}
$$

où $\langle b|U| c\rangle$ et $\langle a|U| c\rangle$ sont les éléments de matrice de champ cristallin, supposés indépendants de $k_{1}$ et $k_{2}$.

Lorsque : $\theta_{\mathrm{D}} / T>10$, on obtient le temps de relaxation $T_{1}^{R}$ associé aux processus Raman "virtuels", pour un doublet de Kramers dégénéré :

$$
\begin{aligned}
\frac{1}{T_{1}^{\mathrm{R}}}= & \frac{9}{4 \pi^{3} \hbar^{2} \rho^{2} v^{10}} \times \\
& \times \frac{|\langle b|U| c\rangle|^{2}|\langle a|U| c\rangle|^{2}}{\omega_{a c}^{4}} 8 !\left(\frac{k_{\mathrm{B}} T}{\hbar}\right)^{9} .
\end{aligned}
$$

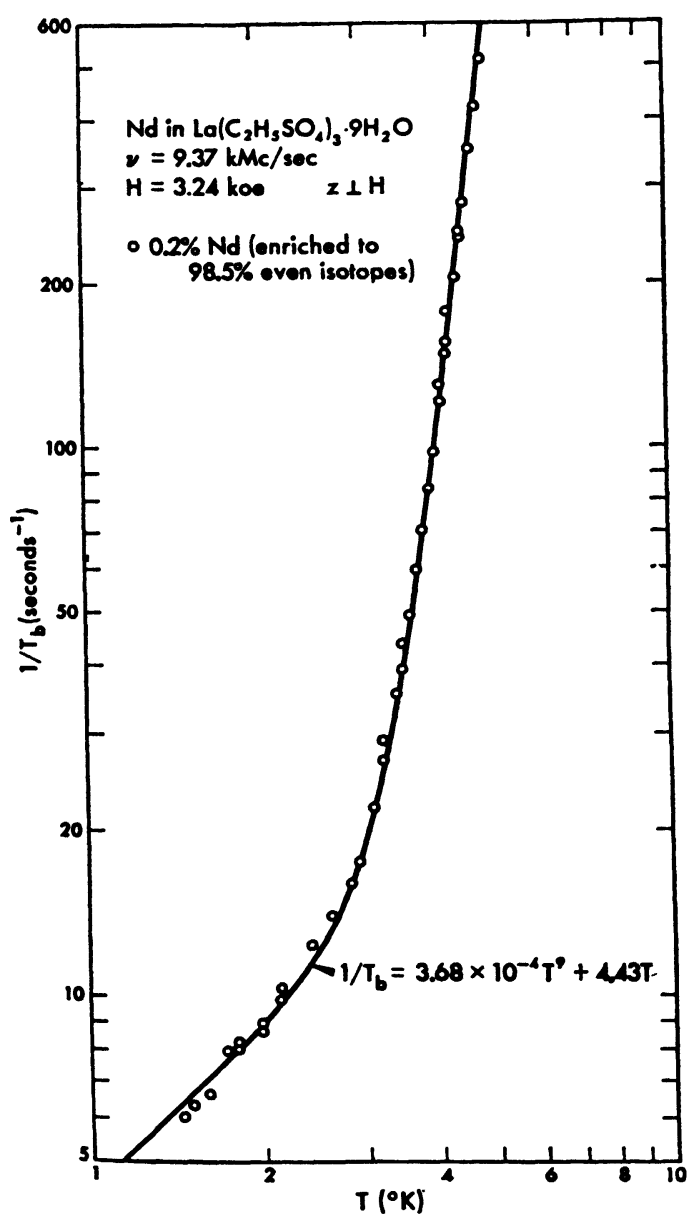

Fig. 23. - (D'après [7]) Variation thermique de la fréquence de relaxation spin-phonons $1 / T_{1}$ pour $\mathrm{Nd}^{3+}$ dilué à $0,2 \%$ at. dans LaES (voir texte).

[(After [7]) Thermal variation of the spin-phonon relaxation rate $1 / T_{1}$ for $\mathrm{Nd}^{3+}$ diluted at $0.2 \%$ at. in LaES (see text).]
La figure 23 [7] reproduit la variation thermique du temps de relaxation $1 / T_{1}$, mesuré par R.P.E., du doublet fondamental de l'impureté de Kramers $\mathrm{Nd}^{3+}$ dans un sel de lanthane. La loi ajustée montre une superposition de processus direct et Raman.

iii) Les processus «Orbach" (branche acoustique) :

Lorsque le niveau excité de champ cristallin $|c\rangle$ est «à portée des phonons " $\left(\omega_{a c}<\omega_{\mathrm{D}}\right)$, la théorie de perturbation au $1^{\mathrm{er}}$ ordre montre qu'une transition entre $|a\rangle$ et $|b\rangle$ peut être induite par deux processus directs entre $|a\rangle$ et $|c\rangle$ d'une part, entre $|c\rangle$ et $|b\rangle$ d'autre part (cf. Fig. 24).

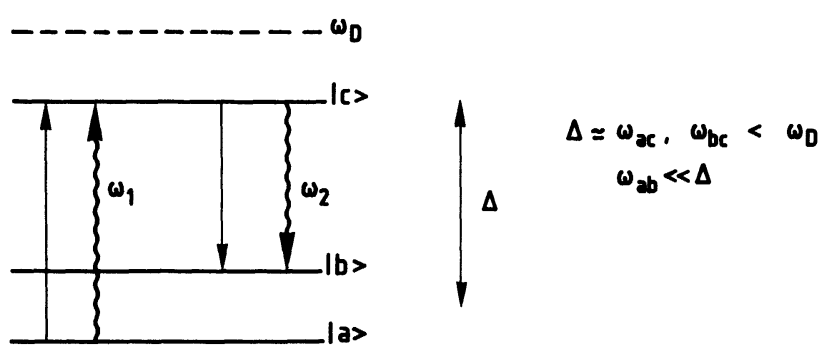

Fig. 24. - Processus Orbach d'absorption entre trois niveaux ioniques $|a\rangle,|b\rangle$ et $|c\rangle$ (phonons acoustiques).

[Orbach absorption process between three ionic levels $|a\rangle,|b\rangle$ and $|c\rangle$ (acoustic phonons).]

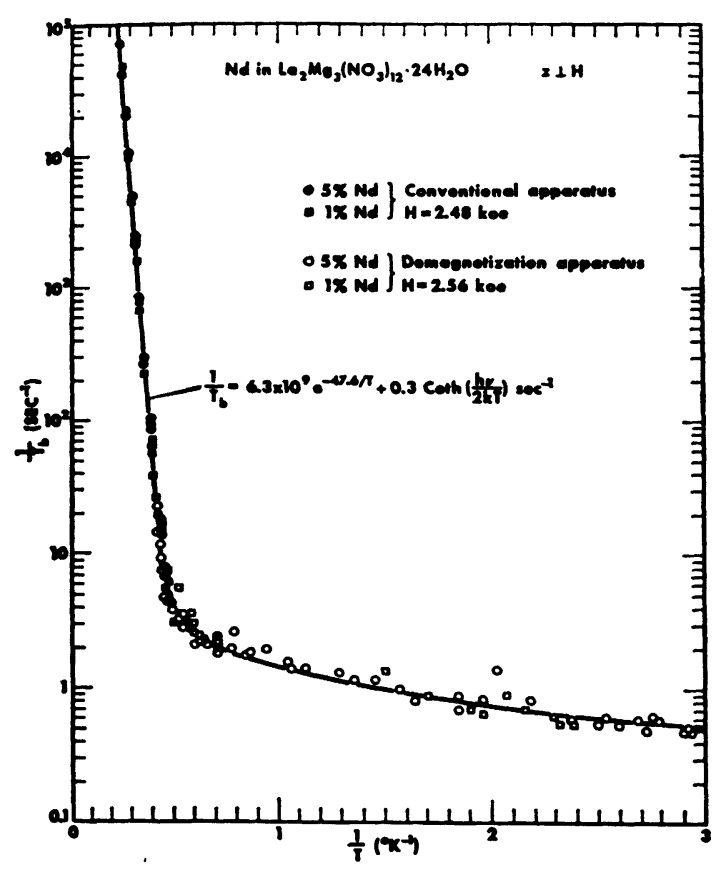

Fig. 25. - (D'après [68]) Variation thermique de la fréquence de relaxation spin-phonons $1 / T_{1}$ pour $\mathrm{Nd}^{3+}$ dilué dans des monocristaux de LaMN dans le domaine de température : $0,3 \mathrm{~K}<T<4,3 \mathrm{~K}$.

[(After [68]) Thermal variation of the spin-phonon relaxation rate $1 / T_{1}$ for $\mathrm{Nd}^{3+}$ diluted in LaMN single crystals in the temperature range : $0.3 \mathrm{~K}<T<4.3 \mathrm{~K}$.] 
La dépendance thermique de $1 / T_{1}$ associé au processus Orbach se déduit des dépendances thermiques des densités spectrales $J_{\mathrm{a}}^{\mathrm{d}}(\Delta)$ et $J_{\mathrm{e}}^{\mathrm{d}}(\Delta)$ des processus directs (formules (102) et (104)); lorsque : $k_{\mathrm{B}} T \ll \Delta$, on a :

$$
J_{\mathrm{a}}^{\mathrm{d}}(\Delta) \propto \frac{1}{\mathrm{e}^{\Delta / k_{\mathrm{B}} T}-1} \simeq \mathrm{e}^{-\Delta / k_{B} T}
$$

et : $J_{\mathrm{e}}^{\mathrm{d}}(\Delta)$ est indépendant de la température.

Donc :

$$
\frac{1}{T_{1}^{\text {or }}} \propto \frac{1}{\mathrm{e}^{\Delta / k_{B} T}-1} \simeq \mathrm{e}^{-\Delta / k_{B} T}
$$

La figure 25 [68] montre la superposition de processus directs et Orbach. La présence de tels processus rend possible la mesure de l'écart de champ cristallin $\Delta$ à des températures telles que : $k_{\mathrm{B}} T \ll \Delta$.

iv) Les processus " Raman" (branches optiques) :

Lorsqu'il est possible de réaliser des mesures de relaxation spin-phonon à haute température, par exemple sur un ion $S$, les branches optiques de phonons, situées à environ : $\omega_{\mathrm{E}} \sim 1000 \mathrm{~K}$, peuvent contribuer aux processus Raman (Fig. 26), lorsque la largeur $\delta$ de la branche optique est supérieure à $\omega_{a b}$ (conservation de l'énergie).

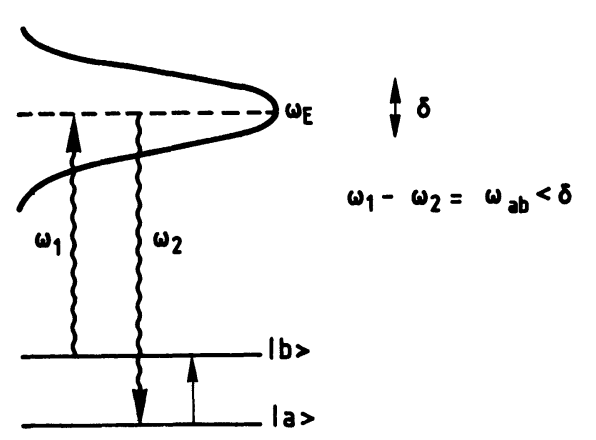

Fig. 26. - Processus Raman « réel " d'absorption à deux phonons optiques entre les niveaux ioniques $|a\rangle$ et $|b\rangle$.

["Real " absorption Raman process with two optical phonons between the ionic levels $|a\rangle$ and $|b\rangle$.]

L'intégrale (110) donnant la probabilité d'absorption devient, en considérant que : $g_{\mathrm{E}}(\omega) \propto \delta\left(\omega-\omega_{\mathrm{E}}\right)$, et en négligeant $\omega_{a b}$ devant $\omega_{\mathrm{E}}$ :

$$
\begin{aligned}
& W_{a \rightarrow b}^{\mathrm{R}} \propto \int_{\text {br. optique }} \mathrm{d} \omega \delta\left(\omega-\omega_{\mathrm{E}}\right) \frac{\omega^{2}}{v^{4}} n(\omega, T) \times \\
& \times[n(\omega, T)+1]\left|\left\langle b\left|U_{k_{1} k_{2}}\right| a\right\rangle\right|^{2}, \\
& W_{a \rightarrow b}^{\mathrm{R}} \propto \frac{\left|\left\langle b\left|U_{2}\right| a\right\rangle\right|^{2}}{v^{4}} \omega_{\mathrm{E}}^{2} n\left(\omega_{\mathrm{E}}, T\right) \times
\end{aligned}
$$$$
\times\left[n\left(\omega_{\mathrm{E}}, T\right)+1\right],
$$

c'est-à-dire :

$$
W_{a \rightarrow b}^{\mathrm{R}} \propto \frac{1}{\sinh ^{2} \frac{\omega_{\mathrm{E}}}{2 k_{\mathrm{B}} T}} .
$$

L'examen de la dépendance thermique de $1 / T_{1}=$ $2 W^{\mathrm{R}}$ permet alors de mesurer l'énergie $\omega_{\mathrm{E}}$ des branches optiques. Par exemple les mesures de la largeur de raie R.P.E. de l'ion $S \mathrm{Mn}^{2+}$ entre $100 \mathrm{~K}$ et $1000 \mathrm{~K}$ [69] (Fig. 27) montrent une superposition de processus Raman « acoustiques » en $T^{7}$ (la symétrie de Kramers est brisée par le champ magnétique appliqué, qui est du même ordre de grandeur que le champ cristallin) et " optiques" suivant la loi donnée par (118). On voit sur la figure 27, que l'énergie ajustée de la branche de phonons optiques est : $\omega_{\mathrm{E}} \simeq 425 \mathrm{~K}$, et que la fréquence de relaxation $1 / T_{1}$ à $T=1000 \mathrm{~K}$ est très «lente " : $1 / T_{1}(1000 \mathrm{~K}) \sim 4 \times 10^{7} \mathrm{~Hz}$.

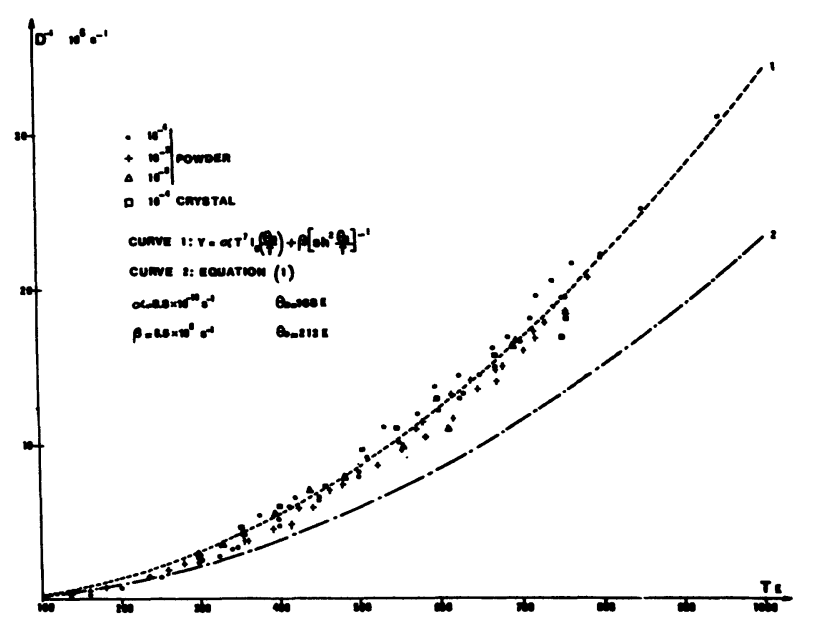

Fig. 27. - (D'après [69]) Variation thermique de la fréquence de relaxation spin-phonons $1 / T_{1}$ pour $\mathrm{Mn}^{2+}$ dilué dans $\mathrm{ZnS}$. La courbe (2) est un ajustement à l'aide des seuls processus Raman acoustiques $\left(T^{7}\right)$.

[(After [69]) Thermal variation of the spin-phonon relaxation rate $1 / T_{1}$ for $\mathrm{Mn}^{2+}$ diluted in $\mathrm{ZnS}$. Curve (2) is a fit with acoustic Raman processes alone $\left(T^{7}\right)$.]

Signalons enfin que, lorsque le multiplet ionique fondamental comporte plus de deux niveaux proches en énergie, le processus Raman conduit à un terme supplémentaire en $T^{5}$ dans l'expression de $1 / T_{1}$ [70]. Cette situation est observée dans le cas des ions $S$. Un terme en $T^{5}$ a été également mis en évidence (par des mesures de susceptibilité alternative) dans le cas de l'ion non de Kramers $\mathrm{Ni}^{++}$, comportant un triplet fondamental $(S=1)$ quasi dégénéré [71]. L'importance relative des termes en $T^{5}$ et en $T^{7}$ pour les ions $S$ est discutée dans la référence [72].

5.2 Relaxation par Les électrons De CONDUCTION. - Nous allons considérer deux états ioniques $|a\rangle$ et $|b\rangle$, séparés par une énergie $\hbar \omega_{a b}$, provenant 
d'un ion de terre rare en impureté dans une matrice métallique. Le spin vrai $\mathbf{S}$ de cet ion est couplé par échange au spin $\mathbf{s}$ d'un électron de conduction

$$
\mathscr{H}_{1}=-2 J_{\mathrm{sf}} \mathrm{SS},
$$

où $J_{\mathrm{sf}}$ est la constante d'échange (en principe positive pour l'échange " atomique ").

A basse température, on a la relation suivante entre les quantités $\mathbf{S}$ (spin vrai) et $\mathbf{J}$ (moment angulaire) : $\mathbf{S}=\left(g_{\mathrm{J}}-1\right) \mathbf{J}$, où $g_{\mathrm{J}}$ est le facteur de Landé de l'ion.

L'interaction d'échange isotrope s'écrit alors :

$$
\mathscr{H}_{1}=-2\left(g_{\mathrm{s}}-1\right) J_{\mathrm{sf}} \mathbf{s . J} .
$$

La probabilité de diffusion inélastique d'un électron de conduction par l'impureté se calcule par la règle d'or de Fermi (cf. Fig. 28), par exemple pour une transition d'absorption :

$$
\begin{aligned}
& W_{a \rightarrow b}^{\mathbf{k} \sigma \rightarrow \mathbf{k}^{\prime} \sigma^{\prime}}=\frac{2 \pi}{\hbar^{2}} \mid\left\langle\mathbf{k}^{\prime}, \sigma^{\prime}, \text { inoccupé } ;\right. \\
& \quad b\left|\mathscr{H}_{1}\right| \mathbf{k}, \sigma, \text { occupé } ; a>\left.\right|^{2} \delta\left(\omega_{a b}-\omega_{k}+\omega_{k^{\prime}}\right),
\end{aligned}
$$

où : $\hbar \omega_{k}=\hbar^{2} k^{2} / 2 m$ est l'énergie cinétique de l'électron itinérant dans le cristal. Soit, en remplaçant $H_{1}$ par l'expression (121) :

$$
\begin{aligned}
\underset{a \rightarrow b}{\mathbf{k} \sigma \rightarrow \mathbf{k}^{\prime} \sigma^{\prime}}=\frac{2 \pi}{\hbar^{2}} & \cdot 4 J_{\mathrm{sf}}^{2}\left(g_{\mathrm{J}}-1\right)^{2} \times \\
& \times\left|\sum_{i}\left\langle\mathbf{k}^{\prime}, \sigma^{\prime}\left|s_{i}\right| \mathbf{k} \sigma\right\rangle\left\langle b\left|J_{i}\right| a\right\rangle\right|^{2} \\
& \times \delta\left(\omega_{a b}-\omega_{k}+\omega_{k^{\prime}}\right)
\end{aligned}
$$

où $\left|\mathbf{k}^{\prime}, \sigma^{\prime}\right\rangle$ est inoccupé et $|\mathbf{k} \sigma\rangle$ occupé.

$$
\text { Si : } f\left(\omega_{k}\right)=\frac{1}{\exp \left(\frac{\hbar \omega_{k}-E_{\mathrm{F}}}{k_{\mathrm{B}} T}\right)-1} \text { est la distribu- }
$$

tion de Fermi, on obtient, pour la probabilité totale de transition :

$$
\begin{aligned}
W_{a \rightarrow b}=\frac{8 \pi}{\hbar^{2}} J_{\mathrm{sf}}^{2}\left(g_{\mathrm{j}}-1\right)^{2} \times \\
\left.\times \sum_{\sigma, \sigma^{\prime}}\left|\sum_{i}\left\langle\sigma^{\prime}\left|s_{i}\right| \sigma\right\rangle\left\langle b\left|J_{i}\right| a\right\rangle\right|^{2}\right\} \\
\times \int_{0}^{\infty} \mathrm{d} \omega_{k} \mathrm{~d} \omega_{k^{\prime}} f\left(\omega_{k}\right)\left[1-f\left(\omega_{k^{\prime}}\right)\right] \\
\times n\left(\omega_{k}\right) n\left(\omega_{k^{\prime}}\right) \delta\left(\omega_{a b}-\omega_{k}+\omega_{k^{\prime}}\right) .
\end{aligned}
$$

Dans cette formule, $n\left(\omega_{k}\right)$, qui représente la densité d'états électroniques par direction de spin de la bande de conduction à l'énergie $\omega_{k}$ est proportionnel à $\sqrt{\omega_{k}}$ dans l'approximation des électrons libres.

Après intégration sur $\omega_{k^{\prime}}$, on obtient [29], en consi-

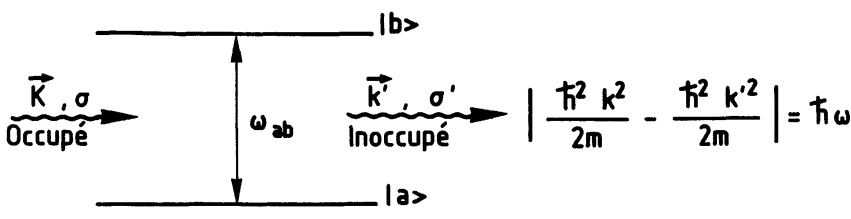

Fig. 28. - Diffusion inélastique d'un électron de conduction $(\mathbf{k}, \sigma) \rightarrow\left(\mathbf{k}^{\prime}, \sigma^{\prime}\right)$ par un ion paramagnétique (états $|a\rangle$ et $|b\rangle)$.

[Inelastic scattering of a conduction electron $(\mathbf{k}, \sigma) \rightarrow$ $\left(\mathbf{k}^{\prime}, \sigma^{\prime}\right)$ by a paramagnetic ion (states $|a\rangle$ and $\left.|b\rangle\right)$.]

dérant que $n\left(\omega_{k}\right)$ et $n\left(\omega_{k^{\prime}}\right)$ varient lentement au voisinage de $E_{\mathrm{F}}$ :

$$
\begin{aligned}
& W_{a \rightarrow b}=\frac{2 \pi}{\hbar^{2}} J_{\mathrm{sf}}^{2}\left(g_{\mathrm{J}}-1\right)^{2}\left\{2\left|\left\langle b\left|J_{\mathrm{z}}\right| a\right\rangle\right|^{2}+\right. \\
& \left.\quad \times\left|\left\langle b\left|J_{+}\right| a\right\rangle\right|^{2}+\left|\left\langle b\left|J_{-}\right| a\right\rangle\right|^{2}\right\} J_{\uparrow}\left(\omega_{a b}\right),
\end{aligned}
$$

où la densité spectrale d'absorption $J_{\uparrow}\left(\omega_{a b}\right)$ vaut :

$J_{\uparrow}\left(\omega_{a b}\right)=\hbar^{2} n^{2}\left(E_{\mathrm{F}}\right) \int_{0}^{\infty} \mathrm{d} \omega f(\omega)\left[1-f\left(\omega-\omega_{a b}\right)\right]$.

L'intégrale se calcule par le changement de variable : $\mu=\frac{\hbar \omega-E_{\mathrm{F}}}{k_{\mathrm{B}} T}$.

On obtient alors :

$$
J_{\uparrow}\left(\omega_{a b}\right)=n^{2}\left(E_{\mathrm{F}}\right) \frac{\hbar^{2} \omega_{a b}}{\mathrm{e}^{n \omega_{a b} / k_{\mathrm{B}} T}-1} .
$$

La densité spectrale d'émission $J_{\downarrow}\left(\omega_{a b}\right)$ s'obtient simplement :

$$
J_{\downarrow}\left(\omega_{a b}\right)=\mathrm{e}^{h \omega_{a b} / k_{B} T} J_{\uparrow}\left(\omega_{a b}\right) .
$$

Lorsque : $\hbar \omega_{a b} / k_{\mathrm{B}} T \rightarrow 0$, on voit que $J_{\uparrow}\left(\omega_{a b}\right)$ et $J_{\downarrow}\left(\omega_{a b}\right)$ tendent vers une limite commune $n^{2}\left(E_{\mathrm{F}}\right) k_{\mathrm{B}} T$ indépendante de $\omega_{a b}$. Ceci correspond à la diffusion élastique sur un doublet de Kramers dégénéré; la fréquence de relaxation s'écrit dans ce cas :

$$
\begin{aligned}
W^{\text {E.C. }}=W_{a \rightarrow b}= & W_{b \rightarrow a}=\frac{2 \pi}{\hbar} J_{\mathrm{sf}}^{2}\left(g_{\mathrm{J}}-1\right)^{2} \times \\
& \times n^{2}\left(E_{\mathrm{F}}\right)\left|\left\langle b\left|J_{+}\right| a\right\rangle\right|^{2} k_{\mathrm{B}} T .
\end{aligned}
$$

Comme le spin fictif $\mathbf{S}^{\prime}$ du doublet vaut $: \mathbf{S}^{\prime}=\frac{g_{\mathrm{J}}}{g} \mathbf{J}$, on a :

$$
\begin{gathered}
W^{\text {E.c. }=} \frac{1}{2 T_{2}}=\frac{1}{2 T_{1}}=\frac{2 \pi}{\hbar} J_{\mathrm{sf}}^{2} \alpha^{2} n^{2}\left(E_{\mathrm{F}}\right) k_{\mathrm{B}} T \\
\left(\alpha=g \frac{g_{\mathrm{J}}-1}{g_{\mathrm{J}}}\right) .
\end{gathered}
$$


Cette loi de variation thermique linéaire, valable lorsque $\hbar \omega_{a b} \ll k_{\mathrm{B}} T$ est appelée loi de Korringa; elle a été obtenue initialement pour la relaxation nucléaire dans les métaux [73], sous l'influence de l'interaction de contact avec les électrons de conduction : $\mathscr{H}_{\mathrm{c}}=A_{\mathrm{c}} \mathbf{s I} \delta(\mathbf{r})$,où I est le spin nucléaire.

Remarquons que la diffusion élastique sur un doublet de Kramers s'accompagne du renversement du spin de l'électron diffusé (seul le terme en $\left\langle\mathbf{k}^{\prime}, \sigma\right.$, $\left.b\left|s_{-} J_{+}\right| \mathbf{k},-\sigma, a\right\rangle$ est non nul).

Dans la limite opposée, lorsque $\hbar \omega_{a b} \gg k_{\mathrm{B}} T$, les deux densités spectrales $J_{\uparrow}$ et $J_{\downarrow}$ ont des comportements txès différents (cf. Fig. 29, où on a tracé en fait : $\left.J(\omega) / \hbar n^{2}\left(E_{\mathrm{F}}\right)\right): J_{\uparrow}(\omega)$ tend exponentiellement vers 0 , ce qui signifie que lorsque l'énergie de la transition ionique à effectuer est beaucoup plus grande que la " plage d'excitation " $k_{\mathrm{B}} T$ des électrons de conduction, ceux-ci ne peuvent fournir l'énergie nécessaire. $\mathrm{Par}$ contre, $J_{\downarrow}(\omega)$ tend asymptotiquement vers $\hbar^{2} \omega n^{2}\left(E_{\mathrm{F}}\right)$, c'est-à-dire devient indépendant de la température : l'ion peut fournir de l'énergie avec une probabilité finie à la bande de conduction, à condition qu'il se trouve dans l'état excité $|b\rangle$, dont la probabilité $\mathrm{e}^{-h \omega_{a b} / k_{B} T}$ est également exponentiellement faible à l'équilibre de Boltzmann.

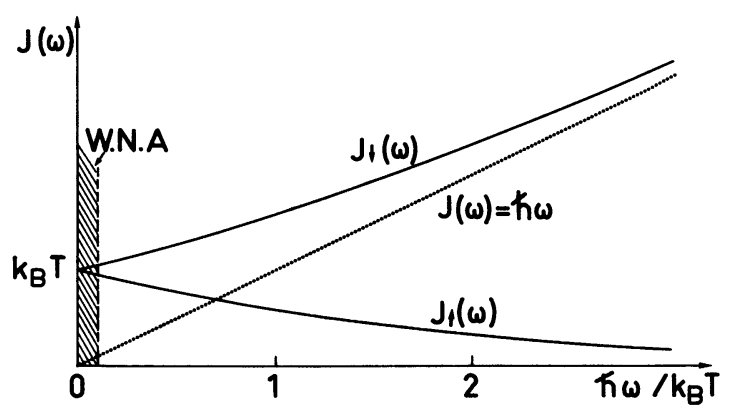

Fig. 29. - Densités spectrales d'absorption $J_{\uparrow}(\omega) / \hbar n^{2}\left(E_{\mathrm{F}}\right)$ (expression (125)) et d'émission $J_{\downarrow}(\omega) / \hbar n^{2}\left(E_{F}\right)$ (expression (126)) associées à la diffusion des électrons de conduction par un ion à deux niveaux électroniques. La région hachurée correspond au domaine de validité de l'approximation du spectre blanc : $\hbar \omega \ll k_{\mathrm{B}} T$.

[Spectral densities for absorption $J_{\uparrow}(\omega) / \hbar n^{2}\left(E_{\mathrm{F}}\right)$ (expression (125)) and for emission $J_{\downarrow}(\omega) / \hbar n^{2}\left(E_{\mathrm{F}}\right)$ (expression (126)) associated with scattering of a conduction electron by a two-level paramagnetic system. The hatched region corresponds to the zone where the white noise approximation is valid : $\hbar \omega \ll k_{\mathrm{B}} T$.]

Les mesures de la dépendance thermique de la largeur de la raie R.P.E. d'impuretés de Kramers $\left(\mathrm{Dy}^{3+}, \mathrm{Er}^{3+}, \mathrm{Yb}^{3+}\right)$ dans les métaux ont très bien vérifié les prédictions représentées par les expressions (124) et (127). Lorsqu'un état $|c\rangle$ de champ cristallin est proche du doublet fondamental, on observe, superposée à la loi de Korringa (127) décrivant la relaxation au sein du fondamental, une dépendance thermique de type (124), due à des processus dits de " Hirst-Orbach ", dans lesquels un électron de conduction est diffusé inélastiquement, l'ion transitant de $|a\rangle$ ou $|b\rangle$ vers $|c\rangle$ [27].

Dans ce cas, on $\mathrm{a}$, en première approximation, et lorsque : $\hbar \omega_{a b} \ll k_{\mathrm{B}} T \ll \hbar \omega_{a c}$ :

$$
\frac{1}{T_{1}}=\frac{1}{T_{2}}=\frac{1}{T_{2}^{\text {is }}}+W_{a \rightarrow c}+W_{b \rightarrow c}
$$

où $1 / T_{2}^{\text {is }}$ représente la contribution à $1 / T_{2}$ provenant du doublet fondamental supposé isolé. Ceci conduit à une variation thermique du type :

$$
\frac{1}{T_{2}}=A T+B \frac{1}{\mathrm{e}^{4 / k_{\mathrm{B}} T-1}},
$$

où : $\Delta \simeq \omega_{a c} \simeq \omega_{b c}$.

On remarque que la dépendance thermique des processus de "Hirst-Orbach " est identique à celle des processus Orbach induits par les phonons, et qu'on ne peut pas en principe les distinguer (la relaxation par phonons est évidemment présente dans les substances métalliques). La figure 30 illustre une telle dépendance thermique dans le cas de $\mathrm{Nd}^{3+}$ dilué dans $\operatorname{LaIr}_{2}$ [74].

Pour terminer ce paragraphe, on peut conclure que les mécanismes de relaxation électronique induits par les phonons ou les électrons de conduction se prêtent bien à la vérification expérimentale des modèles théoriques utilisés pour calculer les dépen-

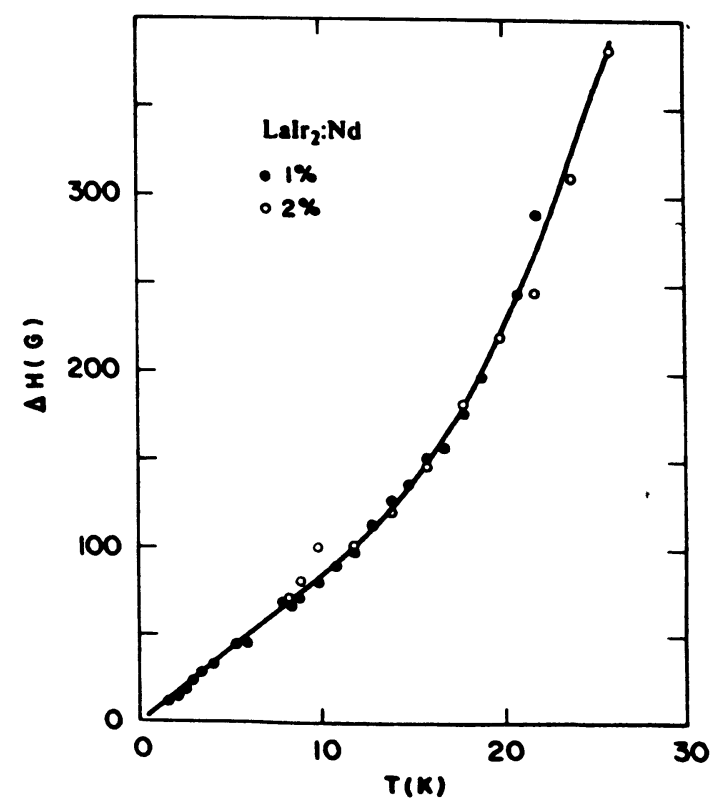

Fig. 30. - (D'après [74]) Variation thermique de la largeur de la raie R.P.E. de $\mathrm{Nd}^{3+}$ dans $\mathrm{LaIr}_{2}$. La ligne continue est un ajustement à une loi du type (129), conduisant à une valeur de l'écart de champ cristallin : $\Delta=94 \mathrm{~K}$.

[(After [74]) Thermal variation of the E.P.R. linewidth of $\mathrm{Nd}^{3+}$ in $\mathrm{LaIr}_{2}$. The full line is a fit to a law of the type (129), leading to a value of the crystal field splitting : $\Delta=94 \mathrm{~K}$.] 
dances thermiques des fréquences de relaxation correspondantes. L'ajustement de ces lois théoriques donne accès à deux types principaux de constantes microscopiques : les énergies de champ cristallin et les constantes de couplage d'échange entre électrons localisés (4f) et électrons itinérants. Toutes les illustrations données dans ce paragraphe montrent que, dans ce domaine, l'accord entre théorie et expérience est très satisfaisant.

\section{La relaxation « spin-spin ».}

Jusqu'à présent, notre intérêt a porté principalement sur le comportement dynamique d'un ion individuel, soumis à l'interaction de relaxation considérée comme une perturbation. Cette description est valable tant que l'on peut négliger les interactions d'échange $\left(\mathfrak{H}_{\text {ech }}\right)$ ou dipolaires magnétiques $\left(\mathscr{H}_{\text {dip }}\right)$, par exemple dans une substance où la concentration en ions magnétiques résonnants est faible. Par contre, dans les corps magnétiquement concentrés, et lorsque les interactions $\mathcal{H}_{\text {ech }}$ et/ou $\mathcal{H}_{\text {dip }}$ sont importantes, les niveaux d'énergie du système de spins, et donc les signaux mesurés par les différentes techniques, sont déterminés par le hamiltonien total :

$$
\mathscr{H}=\mathscr{H}_{0}+\mathscr{H}_{\mathrm{ech}}+\mathscr{H}_{\mathrm{dip}},
$$

où $\mathcal{H}_{0}$ comprend l'interaction de champ cristallin et de spin-orbite (ou l'interaction hyperfine) ainsi que l'interaction Zeeman si l'on applique un champ magnétique.

L'interaction d'échange $\mathfrak{H}_{\mathrm{ech}}$ (supposée isotrope) s'écrit :

$$
\mathfrak{H}_{\mathrm{ech}}=\frac{1}{2} \sum_{i, j} \mathfrak{J}_{i j} \mathbf{S}_{i} \cdot \mathbf{S}_{j}
$$

où $\mathbf{S}_{i}$ et $\mathbf{S}_{j}$ sont les spins vrais des ions $i$ et $j$.

Pour les terres rares, où $\mathbf{J}$ est un bon nombre quantique, on a :

$$
\mathscr{H}_{\mathrm{ech}}=\frac{1}{2}\left(g_{\mathrm{J}}-1\right)^{2} \sum_{i, j} \mathfrak{J}_{i j} \mathbf{J}_{i} \cdot \mathbf{J}_{j} .
$$

L'interaction dipolaire magnétique s'écrit en fonction des moments magnétiques $\mathbf{m}_{i}$ de chaque ion $\left(r_{i j}\right.$ est la distance entre les ions $\underline{i}$ et $\underline{j}$ ) :

$\mathcal{H}_{\mathrm{dip}}=\frac{1}{2} \sum_{i j} \frac{1}{r_{i j}^{3}}\left[\mathbf{m}_{i} \cdot \mathbf{m}_{j}-\frac{3\left(\mathbf{m}_{i} \cdot \mathbf{r}_{i j}\right)\left(\mathbf{m}_{j} \cdot \mathbf{r}_{i j}\right)}{r_{i j}^{2}}\right]$.

Traditionnellement, on décompose $\mathfrak{H}_{\text {ech }}$ et $\mathfrak{H}_{\text {dip }}$ en 3 sortes de termes désignés par: $A$ et $B, C$ et $D\left(C=D^{*}\right)$, et $E$ et $F\left(E=F^{*}\right)$. Les quantités $A$ et $B$ contiennent respectivement les termes en : $S_{i \mathbf{z}} S_{j \mathrm{z}}$ et : $S_{+i} S_{-j}+$ $S_{-i} S_{+j}$. L'interaction d'échange isotrope ne contient évidemment que des termes du type $A$ et $B$.
On peut montrer que : $\left[\mathrm{A}+\mathrm{B}, \sum_{i} S_{i \mathrm{z}}\right]=0$; ceci signifie que ces termes "séculaires " de l'interaction spin-spin ne modifient pas l'énergie Zeeman :

$$
E=-g \mu_{\mathrm{B}} H_{0} \sum_{i} S_{i \mathrm{z}},
$$

au sein d'un système de spins identiques.

La quantité $C$ (resp. D) contient les termes en $S_{i \mathrm{z}} S_{j+}$ (resp. $S_{i \mathrm{z}} S_{j-}$ ), et $E$ (resp. $F$ ) les termes en $S_{+i} S_{+j}\left(\right.$ resp. $S_{-i} S_{-j}$ ).

On pourra trouver l'expression de ces différents termes pour l'interaction dipolaire dans la référence [42].

Ces interactions spin-spin conduisent en général à l'apparition d'un ordre magnétique en dessous d'une certaine température $T_{\mathrm{c}}\left(k_{\mathrm{B}} T_{\mathrm{c}} \sim \mathscr{H}_{\mathrm{ech}}\right.$ ou $\left.\mathcal{H}_{\mathrm{dip}}\right) . \mathrm{La}$ majorité des études de relaxation a lieu dans la zone paramagnétique $\left(T>T_{\mathrm{c}}\right)\left({ }^{9}\right)$. Dans ce domaine de température, les interactions entre moments magnétiques, bien qu'elles n'induisent pas d'aimantation spontanée microscopique, sont à prendre en compte dans le calcul de l'évolution des observables du système. Les signaux mesurés par les techniques spectroscopiques, qui mettent en jeu les opérateurs en représentation de Heisenberg $M(t)$, nécessitent le calcul des fonctions de corrélations :

$$
G(t)=\left\langle M(t) M^{+}(0)\right\rangle=\left\langle\mathrm{e}^{-i 3 t} M \mathrm{e}^{i j t} M^{+}\right\rangle,
$$

où $\mathcal{H}$ représente le hamiltonien du système résonnant, ainsi que les diverses interactions de ce dernier avec son environnement.

Le calcul de $G(t)$ en présence d'interaction entre les spins est en pratique irréalisable, et, de fait, ce problème n'est pas résolu à l'heure actuelle. On a donc recours, pour calculer l'influence des interactions spin-spin sur les formes de raies, à diverses approximations, qui dépendent de chaque situation expérimentale.

Il convient de signaler ici une différence importante, dans le cas d'une assemblée de moments magnétiques identiques, entre les signaux mesurés en R.P.E. (ou R.M.N.) et en susceptibilité alternative d'une part, et par spectroscopie Mössbauer d'autre part.

Dans le cas de la R.P.E., l'interaction avec le champ magnétique de radiofréquence s'écrit :

$\mathscr{H}_{\mathrm{rf}}=-\left(\sum_{i} \mathbf{m}_{i}\right) \mathbf{h} \cos \omega t=-\mathbf{m h} \cos \omega t$,

où $\mathbf{m}$ est l'aimantation totale des ions résonnants. $\mathrm{La}$ R.P.E. est donc une technique sensible au mouvement

(9) Voir cependant le $\S 6.3 .2$ pour l'observation des phénomènes de relaxation dans la zone ordonnée à l'aide de la spectrométrie Mössbauer. 
du moment magnétique total, et les fonctions de corrélation $G(t)$ correspondantes valent :

$$
G_{i}(t)=\left\langle\mathrm{e}^{-i\left(-m_{z} H_{0}+J_{s s}\right) t} m_{\mathrm{i}} \mathrm{e}^{i\left(-m_{z} H_{0}+S_{s s}\right) t} m_{\mathrm{i}}^{+}\right\rangle,
$$

où : $\mathscr{H}_{\mathrm{ss}}=\mathscr{H}_{\mathrm{dip}}+\mathscr{H}_{\mathrm{ech}}$ (et des expressions analogues pour $\chi_{\|}(\omega)$ et l'aimantation nucléaire en R.M.N.).

En spectroscopie Mössbauer, l'interaction entre le noyau et le rayonnement électromagnétique est "locale", l'opérateur mis en jeu étant le moment $2^{l}$-polaire « dynamique " $T_{l}^{M}$ d'un noyau. La fonction de corrélation correspondante est :

$$
G_{M}(t)=\left\langle\mathrm{e}^{-i\left(\mathcal{H}_{\mathrm{hpf}}+\mathfrak{S}_{\mathrm{ss}}\right) t} T_{l}^{M} \mathrm{e}^{i\left(\mathcal{S e}_{\mathrm{hpr}}+\mathcal{H}_{\mathrm{ss}}\right) t} T_{l}^{-M}\right\rangle,
$$

où $\mathfrak{H}_{\mathrm{hpf}}$ représente l'interaction hyperfine entre un noyau résonnant et la couche électronique qui l'entoure.

Dans les approximations utilisées en effet Mössbauer, on pourra donc faire l'hypothèse que, même dans les systèmes concentrés en ions magnétiques, l'effet des interactions spin-spin sur le spectre est bien décrit par des fluctuations individuelles des ions. Par contre, cette approximation ne peut en général pas être utilisée pour calculer la forme de raie R.P.E., sauf lorsque l'ion résonnant est une impureté que l'on suppose sans interactions avec les autres impuretés de même type présentes dans le solide.

Dans la suite de ce paragraphe, nous allons examiner diverses études de relaxation spin-spin, principalement par R.P.E. et spectroscopie Mössbauer. Tout d'abord, nous considérerons l'influence de l'échange isotrope sur les spectres de R.P.E., dans le cas d'ions identiques dont les niveaux d'énergie sont équidistants, puis lorsqu'on observe la résonance d'une impureté substituée dans une matrice d'ions couplés entre eux par échange.

Nous introduirons ensuite brièvement la notion de relaxation croisée, qui est un processus mettant en jeu un petit nombre d'ions dont les énergies de transition sont légèrement différentes. Enfin, nous examinerons divers aspects de la mesure des fréquences de relaxation spin-spin par spectroscopie Mössbauer, dans la zone paramagnétique et dans la zone d'ordre magnétique.

Les exemples choisis représentent des cas où il a été possible d'obtenir une interprétation quantitative des spectres, donnant en général accès aux constantes d'échange inter-ioniques.

6.1 INFLUENCE DE L'ÉCHANGE ISOTROPE SUR LES SPECTRES R.P.E. - $-a$ ) Considérons pour commencer un ensemble de moments magnétiques identiques soumis à un champ magnétique extérieur $H_{0}$, et interagissant entre eux par une interaction spin-spin $\mathfrak{H}_{\mathbf{s s}}$.

Lorsque : $g \mu_{\mathrm{B}} H_{0}=\hbar \omega_{0} \gg \mathscr{H}_{\mathrm{ss}}$, on peut montrer [1] que seule la partie "séculaire" $(A+B)$ de $\mathcal{H}_{\mathrm{ss}}$ contribue à la raie R.P.E. en $\omega=\omega_{0}$. Les termes $C$, $D, E$ et $F$ contribuent à des raies situées en $\omega=0$,
$2 \omega_{0}$ et $3 \omega_{0}$. La forme de la raie en $\omega=\omega_{0}$ en présence d'interactions doit donc être calculée à partir de l'hamiltonien $\mathcal{H}_{\mathrm{ss}}^{\prime}$ "tronqué ":

$$
\mathcal{H}_{\mathrm{ss}}^{\prime}=A+B \text {. }
$$

Il n'est pas possible en général de mener un tel calcul à bien; on peut alors utiliser la "méthode des moments " [1] pour avoir quelques renseignements sur la forme de raie. Pour une courbe (normalisée) $f(\omega)$, ayant un maximum en $\omega_{0}$, le moment d'ordre $n$ est défini par :

$$
M_{n}=\int_{-\infty}^{+\infty}\left(\omega-\omega_{0}\right)^{n} f(\omega) \mathrm{d} \omega
$$

Les moments d'ordre impair sont nuls si la courbe est symétrique par rapport à $\omega_{0}$. En pratique, on ne peut que calculer (ou estimer) les moments d'ordres les plus bas $M_{2}$ et $M_{4}$.

Pour une courbe lorentzienne, on ne peut pas en fait définir de moments, car les intégrales mises en jeu divergent. Cependant, on peut montrer que le rapport : $M_{2} / \sqrt{M_{4}}$ tend vers zéro. Le tableau II donne les valeurs de ce rapport $M_{2} / \sqrt{M_{4}}$, ainsi que l'expression de la demi-largeur à mi-hauteur $\Delta \omega$ pour une courbe gaussienne et pour une lorentzienne " tronquée dans les ailes".

Tableau II. - Valeurs du rapport $M_{2} / \sqrt{M_{4}}$ et de la demi-largeur à mi-hauteur $\Delta \omega$ pour deux types de courbes.

\begin{tabular}{|l|c|c|}
\hline & $M_{2} / \sqrt{M_{4}}$ & $\Delta \omega$ \\
\hline Gaussienne & 0,57 & $1,18 \sqrt{M_{2}}$ \\
\hline $\begin{array}{c}\text { Lorentzienne } \\
\text { «tronquée " }\end{array}$ & $0,1-0,2$ & $0,91 M_{2}^{3 / 2} / \sqrt{M_{4}}$ \\
\hline
\end{tabular}

La forme de raie R.P.E. est proportionnelle à :

$$
I(\omega)=\operatorname{Re} \int_{0}^{\infty} \mathrm{e}^{i \omega t}\left\langle m_{x}(t) m_{x}\right\rangle \mathrm{d} t,
$$

où : $m_{x}(t)=\mathrm{e}^{-i \Im e t} m_{x} \mathrm{e}^{i j e t}, m_{x}$ étant l'aimantation transversale totale des ions résonnants, et :

$$
\mathscr{H}=-m_{\mathrm{z}} H_{0}+\mathscr{H}_{\mathrm{ss}}^{\prime} \text {. }
$$

$\mathcal{H}_{\mathrm{ss}}^{\prime}$ représente ici le hamiltonien " tronqué » $A+B$ de l'interaction spin-spin.

On peut montrer [1] que, à haute température, le second moment associé à cette forme de raie vaut :

$$
M_{2}=-\frac{\operatorname{Tr}\left\{\left[\mathcal{H}_{\mathrm{ss}}^{\prime}, m_{x}\right]^{2}\right\}}{\operatorname{Tr}\left(m_{x}^{2}\right)} .
$$

En présence des seules interactions dipolaires, la 
raie R.P.E. d'un système concentré (en fait, il suffit que la concentration en ions magnétiques identiques soit supérieure à $10 \%$ [75]) est assez proche d'une gaussienne, de largeur : $\Delta \omega_{\text {dip }}=1,18 \sqrt{M_{2}}$.

L'échange isotrope, défini par (131), commute avec :

$$
m_{x}=g \mu_{\mathrm{B}} \sum_{i} S_{i x} .
$$

Il ne contribue donc pas au second moment, tandis qu'il donne une contribution positive au quatrième moment $M_{4}$. Le rapport $M_{2} / \sqrt{M_{4}}$ passe donc de la valeur $\sim 0,57$ (sans l'échange) à une valeur plus faible, qui tend vers zéro lorsque $: \overline{\mathfrak{J}} \gg \Delta \omega_{\text {dip }}$, où $\bar{J}$ est une valeur moyenne de la constante d'échange $J_{i j}$. La forme de raie en présence d'échange se rapproche donc d'une lorentzienne : c'est le "rétrécissement par échange " [52], dont il a déjà été question au $\S$ 4.3. La demi-largeur à mi-hauteur de la lorentzienne vaut alors (lorsque $\overline{\mathfrak{J}} \gg \Delta \omega_{\text {dip }}$ ) :

$$
\Delta \omega \simeq \frac{\left(\Delta \omega_{\text {dip }}\right)^{2}}{\overline{\mathfrak{J}}} \ll \Delta \omega_{\text {dip }}
$$

Lorsque, dans le hamiltonien $\mathscr{H}$ de (136), il faut tenir compte du champ électrostatique cristallin, les énergies de transition entre niveaux électroniques ne sont plus égales (pour un spin $S>1 / 2$ ). Par exemple, le « hamiltonien de spin " de l'ion $\mathrm{Ni}^{++}$, dans un site à symétrie axiale peut s'écrire, lorsque le champ appliqué est parallèle à l'axe cristallin :

$$
\mathscr{H}_{0}=-\mu_{\mathrm{B}} g_{\|} S_{\mathrm{z}} H_{0}-D S_{\mathrm{z}}^{2}, \quad(S=1)
$$

$D$ étant une constante positive décrivant l'écart en énergie à champ nul. On a dans ce cas deux raies R.P.E., et le calcul du second moment pour chaque raie montre que la contribution de l'échange isotrope n'est pas nulle [76].

Donc, dans le cas d'un système d'ions identiques, lorsque l'interaction Zeeman est du même ordre de grandeur que l'interaction de champ cristallin $(D)$, l'influence de l'échange isotrope ne se réduit pas à un " rétrécissement" du spectre, mais peut conduire à un élargissement de chaque raie, qui dépend de la température lorsque : $k_{\mathrm{B}} T \lesssim D$. Ce phénomène d'élargissement par l'échange de la raie R.P.E. a été également observé sur l'ion de Kramers $\mathrm{Er}^{3+}$ dans les alliages métalliques cubiques $\mathrm{Er}_{c} \mathrm{La}_{1-c} \mathrm{Be}_{13}$, où la concentration $c$ varie entre $2 \%$ et $8 \%$ [77]. L'état fondamental de champ cristallin de $\mathrm{Er}^{3+}$ est le doublet $\Gamma_{7}$, séparé d'environ $\Delta_{\mathrm{cc}} \simeq 10 \mathrm{~K}$ du quadruplet excité $\Gamma_{8}$. Les auteurs de la référence [77] développent une nouvelle méthode pour calculer le quatrième moment $M_{4}$ de la raie en présence de l'interaction d'échange isotrope (les interactions dipolaires entre ions $\mathrm{Er}^{3+}$ sont négligeables par rapport à l'échange RKKY), et obtiennent le rapport $M_{2} / \sqrt{M_{4}}$ en fonction de la température. Ils montrent en outre que, lorsque la constante d'échange $\overline{\mathfrak{J}}$ est inférieure

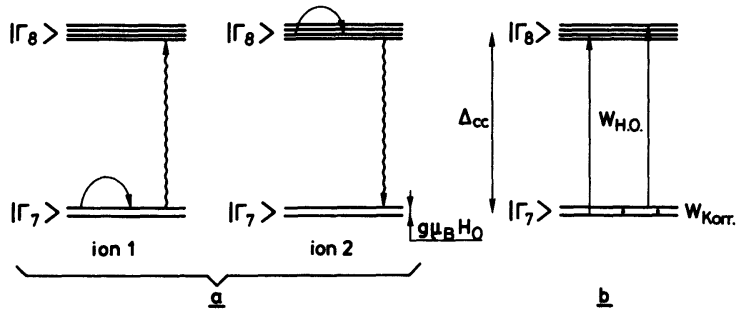

Fig. 31. - a) Représentation schématique des processus à deux ions induits par l'échange isotrope dans $\mathrm{Er}_{\mathrm{c}} \mathrm{La}_{1-\mathrm{c}} \mathrm{Be}_{13} ; \curvearrowright$ : premier terme de (140); \{\} : deuxième terme de (140), correspondant à un "flip-flop " entre états de champ cristallin. b) Processus de relaxation à un ion dû à la diffusion inélastique des électrons de conduction; $W_{\text {Korr }}$ : processus quasi élastiques de Korringa; $W_{\text {H.o. }}$ : processus inélastiques de Hirst-Orbach.

[a) Schematic representation of two-ion processes induced by isotropic exchange in $\mathrm{Er}_{c} \mathrm{La}_{1-c} \mathrm{Be}_{13} ; \curvearrowright$ : first term of (140); \{\} : second term of (140), corresponding to a «flipflop " between crystal field states. $b$ ) Single-ion relaxation process due to inelastic diffusion of conduction electrons; $W_{\text {Korr }}$ : Korringa quasi-elastic process; $W_{\text {H.o. }}$ : HirstOrbach inelastic process.]

à $\Delta_{\text {cc }}$ (ce qui est le cas ici : $\bar{\jmath} \sim 1,5 \mathrm{~K}$ ), les seules contributions aux $2^{\mathrm{e}}$ et $4^{\mathrm{e}}$ moments proviennent des termes suivants (cf. Fig. 31a) :

$$
\left\langle\Gamma_{7}^{i}, \Gamma_{8}^{j}\left|\mathbf{J}_{i} \cdot \mathbf{J}_{j}\right| \Gamma_{7}^{i}, \Gamma_{8}^{j}\right\rangle \text { et }\left\langle\Gamma_{7}^{i}, \Gamma_{8}^{j}\left|\mathbf{J}_{i} \cdot \mathbf{J}_{j}\right| \Gamma_{8}^{i}, \Gamma_{7}^{j}\right\rangle \text {, }
$$

où $\mathbf{J}_{i}$ est le moment angulaire de l'ion $i,\left|\Gamma_{a}^{i}\right\rangle$ représentant la fonction d'onde de champ cristallin $\left|\Gamma_{\alpha}\right\rangle$ de l'ion $i$.

Le calcul du rapport $M_{2} / \sqrt{M_{4}}$ (dépendant de $\bar{\jmath}$ ) donne des valeurs inférieures à 0,25 , ce qui conduit les auteurs à adopter une forme lorentzienne tronquée pour la raie d'absorption. La figure 32 représente la variation thermique de la largeur de raie interprétée comme une superposition de processus Korringa $\left(\Delta H_{\text {Korr }}\right)$ et Hirst-Orbach $\left(\Delta H_{\text {H.o. }}\right)$ dus à la diffusion des électrons de conduction par une impureté $\mathrm{Er}^{3+}$ (cf. Fig. 31b), et d'un terme provenant de l'échange Er-Er :

$$
\Delta H(T)=a+\Delta H_{\text {Korr }}+\Delta H_{\text {H.o. }}+\frac{\hbar}{g \mu_{\mathrm{B}}} 0,91 \frac{M_{2}^{3 / 2}}{\sqrt{M_{4}}},
$$

où $a$ est une largeur résiduelle statique.

La courbe en pointillés sur la figure 32 correspond aux mesures effectuées sur un échantillon dilué (500 ppm de $\mathrm{Er}^{3+}$ ), dans lequel sont seuls présents les processus Korringa et Hirst-Orbach. Les mesures à plus forte concentration (courbes continues) mettent clairement en évidence la contribution à l'élargissement dynamique due à l'échange isotrope, et l'ajuste- 


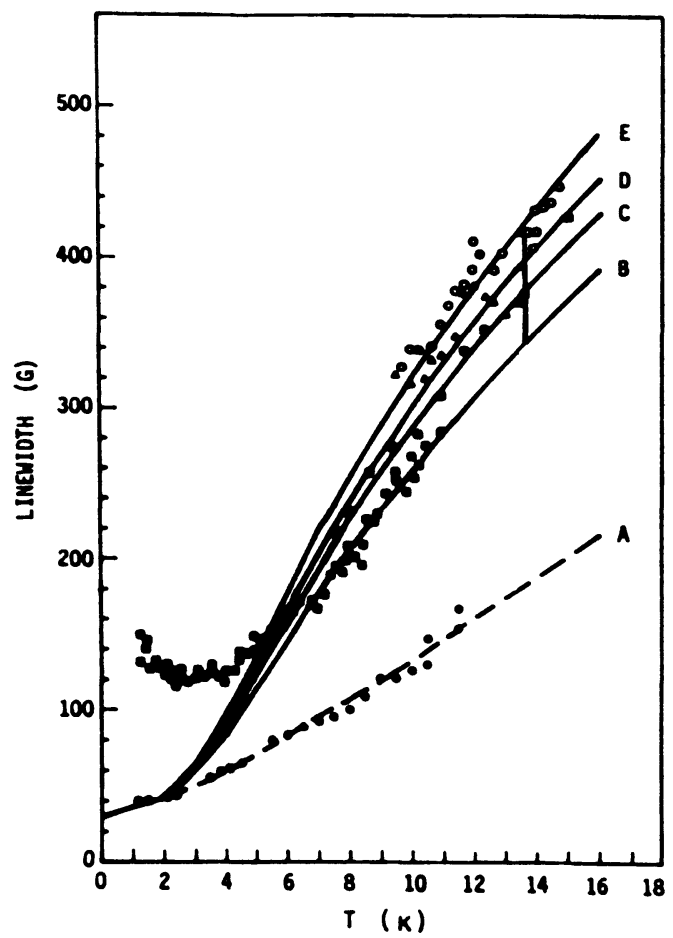

Fig. 32. - (D'après [77]) Variation thermique expérimentale de la largeur de raie R.P.E. de $\mathrm{Er}^{3+}$ dans $\mathrm{LaBe}_{13}$ pour différentes concentrations d'erbium : $500 \mathrm{ppm}$ et $5000 \mathrm{ppm}$; $\square 5 \%$ at. ; $\Delta 7 \%$ at. ; $08 \%$ at. (voir texte).

[(After [77]) Experimental thermal variation of the E.P.R. linewidth of $\mathrm{Er}^{3+}$ in $\mathrm{LaBe}_{13}$ for various erbium concentrations : $500 \mathrm{ppm}$ and $5000 \mathrm{ppm} ; \square 5 \%$ at. ; $\Delta 7 \%$ at. ; O $8 \%$ at. (see text).]

ment à l'aide de l'expression (141) permet d'obtenir la constante d'échange moyenne : $\overline{\mathfrak{J}} \sim 1,5 \mathrm{~K}$.

b) Il est également possible d'observer la résonance d'une impureté substituée (en général l'ion $\mathrm{S} \mathrm{Gd}^{3+}$ ) dans une matrice magnétique dont les ions sont couplés par l'échange. Nous citerons ici les études de la R.P.E. de $\mathrm{Gd}^{3+}$ dans la zone paramagnétique de composés intermétalliques de praséodyme [78] et de thulium [79], dans lesquels l'ion $\mathrm{Pr}^{3+}$ ou $\mathrm{Tm}^{3+}$ possède un état fondamental singulet, donc non magnétique, de champ cristallin. Dans ces composés, la constante d'échange J entre les ions Van-Vleck de la matrice est faible (quelques $\mathbf{K}$ ), et en général supérieure à la constante $J^{\prime}$ d'échange entre l'impureté $\mathbf{G d}^{3+}$ et l'ion hôte. On se trouve donc dans une situation où l'on peut considérer l'échange $\mathrm{Gd}^{3+}$-hôte comme une interaction de relaxation, et la traiter en perturbation (cf. $\S 2.3$ ).

Le hamiltonien $\mathscr{H}_{1}$ vaut :

$$
\mathscr{H}_{1}=\left(\dot{g}_{\mathrm{J}}-1\right) \mathfrak{J}^{\prime} \mathbf{S} \sum_{k=1}^{z_{0}} \mathbf{J}_{k}
$$

où $\mathbf{S}$ est le spin de $\mathrm{Gd}^{3+}, \mathbf{J}_{k}$ le moment angulaire de l'ion de la matrice sur le site $k$ et $z_{0}$ le nombre de premiers voisins $\mathrm{Tm}^{3+}$ ou $\mathrm{Pr}^{3+}$ interagissant par échange avec $\mathrm{Gd}^{3+}$.
La structure fine de champ cristallin de la raie de $\mathrm{Gd}^{3+}$ est, dans la plupart de ces composés, effacée par l'échange Gd-Pr (ou Gd-Tm) sauf dans le cas de $\mathrm{Gd}^{3+}:$ PrSb [78b], où l'on a pu observer une structure résolue à basse température, et suivre sa disparition progressive lorsque la température augmente. En effet, nous allons voir que la fréquence de relaxation transverse $1 / T_{2}$ de $\mathrm{Gd}^{3+}$, due à l'échange (142), augmente avec la température.

Dans l'expression (55), on a, en symétrie cubique :

$$
\begin{aligned}
\frac{1}{T_{2}}=\frac{\pi}{2 \hbar^{2}}\left(J^{\prime}\right)^{2}\left(g_{\mathrm{J}}-1\right)^{2} \times \\
\times\left[2 K_{z z}(0)+K_{x x}\left(\omega_{0}\right)+K_{y y}\left(\omega_{0}\right)\right],
\end{aligned}
$$

où l'on a choisi la direction du champ appliqué comme axe $\mathrm{Oz} ; K_{\alpha \alpha}(\omega)$ est la densité spectrale des fluctuations des ions de la matrice couplés entre eux par l'échange ("bain d'échange ») :

$$
\begin{array}{r}
K_{\alpha \alpha}(\omega)=\int_{-\infty}^{+\infty} \mathrm{d} t \mathrm{e}^{i \omega t}\left\langle\left[\sum_{k=1}^{z_{0}} J_{\alpha k}(t)\right]\left[\sum_{k=1}^{z_{0}} J_{\alpha k}^{+}\right]\right\rangle= \\
=z_{0} \int_{-\infty}^{+\infty} \mathrm{d} t \mathrm{e}^{i \omega t}\left\langle J_{\alpha k}(t) J_{\alpha j}^{+}\right\rangle .
\end{array}
$$

Dans l'expression (143), $\omega_{0}$ est la fréquence de résonance de $\mathrm{Gd}^{3+}$. On peut se rendre compte que ces densités spectrales du bain d'échange augmentent lorsque la température s'élève, en considérant la structure de champ cristallin des ions $\mathrm{Pr}^{3+}$ ou $\mathrm{Tm}^{3+}$ dans les composés étudiés : un singulet fondamental non magnétique, séparé du premier triplet excité par une énergie $\Delta$ (Fig. 33a). On peut écrire la densité

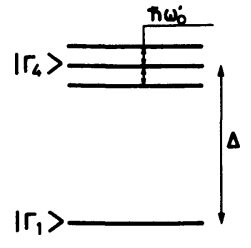

요

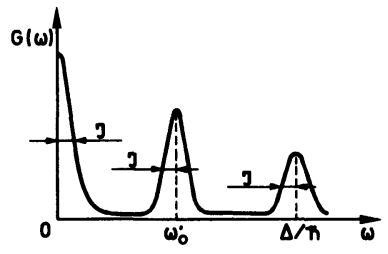

b
Fig. 33. - a) Schéma de champ cristallin de l'ion $\operatorname{Pr}^{3+}$ en symétrie cubique (par exemple, dans $\operatorname{PrP}: \Delta=125 \mathrm{~K}$ [78a]), en présence de champ magnétique

$$
\left(\hbar \omega_{0}^{\prime}=g_{\Gamma_{4}} \mu_{\mathrm{B}} H_{0} \ll \Delta / \hbar\right) .
$$

b) Représentation schématique de la densité spectrale $G(\omega)$ du bain d'échange, dans l'hypothèse où : $\mathfrak{J} \ll \hbar \omega_{0}^{\prime} \ll \Delta$.

[a) Crystal field level scheme of the $\mathrm{Pr}^{3+}$ ion in cubic symmetry (for example, in $\operatorname{PrP}: \Delta=125 \mathrm{~K}[78 a]$ ) in the presence of a magnetic field $\left(\hbar \omega_{0}^{\prime}=g_{\Gamma_{4}} \mu_{\mathrm{B}} H_{0} \ll \Delta / \hbar\right)$. b) Schematic representation of the spectral density $G(\omega)$ of the exchange bath, with the assumption : $J \ll \hbar \omega_{0}^{\prime} \ll \Delta$.] 
spectrale à l'aide d'une fonction $g(t)$ contenant toute la dépendance temporelle :

$$
\begin{aligned}
K_{\alpha \alpha}(\omega)=z_{0}\left\langle J_{\alpha k}^{2}\right\rangle_{T} \int_{-\infty}^{+\infty} \mathrm{d} t \mathrm{e}^{i \omega t} g(t)= \\
\quad=z_{0}\left\langle J_{\alpha k}^{2}\right\rangle_{T} G(\omega),
\end{aligned}
$$

où $\left\langle J_{\alpha k}^{2}\right\rangle_{T}$ est une moyenne thermique sur les états de champ cristallin. La fonction $G(\omega)$ décrit la probabilité que le bain d'échange cède l'énergie $\hbar \omega$ à l'impureté $\mathrm{Gd}^{3+}$ (ou reçoive cette énergie). Dans la limite d'une interaction d'échange $J$ très faible, la fonction $G(\omega)$ possède des pics en $\omega=0$ (fluctuations de $J_{z}$ ), en $\omega=\omega_{0}^{\prime}$ (fluctuations de $J_{x}$ et $J_{y}$ au sein de l'état excité) et en $\omega=\Delta / \hbar$ (fluctuations entre les niveaux de champ cristallin) comme il est montré sur la figure $33 b$. Par contre, lorsque : $\omega_{0}^{\prime} \ll \mathfrak{J} \ll \Delta$, ce qui est le cas rencontré dans ces expériences $(\mathcal{J} \sim$ quelques $\mathrm{K})$, les deux pics "basse fréquence " de $G(\omega)$ se fondent en un seul, de largeur J, et comme : $\omega_{0} \sim \omega_{0}^{\prime} \ll J$, on peut utiliser l'approximation $\mathrm{du}$ spectre blanc pour la densité spectrale, et on obtient le temps de relaxation transverse de $\mathrm{Gd}^{3+}$ :

$$
\frac{1}{T_{2}}=\frac{2 \pi}{\hbar^{2}}\left(\mathfrak{J}^{\prime}\right)^{2}\left(g_{\mathrm{J}}-1\right)^{2} K_{z z}^{\mathrm{EF}}(0) .
$$

$K_{\mathrm{zz}}^{\mathrm{BF}}(0)$ représente la partie «basse fréquence " de la fonction de corrélation, et doit être évaluée à l'aide d'opérateurs " tronqués » (notés $\bar{J}_{z}$, cf. Réf. [78a]) :

$$
K_{z z}^{\mathrm{BF}}(0)=z_{0}\left\langle\bar{J}_{z}^{2}\right\rangle_{T} G(0) \simeq \frac{z_{0}}{J}\left(\sum_{i} p_{i}\left|\left\langle\Gamma_{i}\left|J_{z}\right| \Gamma_{i}\right\rangle\right|^{2}\right),
$$

d'où :

$$
K_{z z}^{\mathrm{BF}}(0) \simeq \frac{z_{0}}{J} \frac{\mathrm{e}^{-\Delta / k_{\mathrm{B}} T}}{Z}\left(\sum_{\alpha}\left|\left\langle\Gamma_{4 \alpha}\left|J_{\mathrm{z}}\right| \Gamma_{4 \alpha}\right\rangle\right|^{2}\right),
$$

dans le cas de $\mathrm{Pr}^{3+}$ (cf. Fig. 33a).

Cette formule montre que $K_{z z}^{\mathrm{BF}}(0)$, donc $1 / T_{2}$ augmente avec la température grâce au peuplement des états excités $\Gamma_{4}$. En fait, ceci n'est vrai qu'à suffisamment basse température; en effet, si on suppose que la densité spectrale normée $G(\omega)$ a une forme gaussienne, on a :

$$
G(0)=\frac{1}{\sqrt{2 \pi M_{2}}}
$$

où $M_{2}$ est le second moment de $G(\omega)$, calculé à l'aide de l'interaction d'échange, convenablement " tronquée ", entre les ions de la matrice. On obtient alors :

$$
K_{z z}^{\mathrm{BF}}(0)=\frac{z_{0}\left\langle\bar{J}_{z}^{2}\right\rangle_{T}}{\sqrt{2 \pi M_{2}(T)}} .
$$

La dépendance en température de la largeur de raie n'est plus simplement due à l'effet de population des niveaux excités, mais également à $\sqrt{M_{2}(T)}$, qui est de l'ordre de $J$, et qui peut montrer une variation thermique importante lorsque : $k_{\mathrm{B}} T \simeq \Delta$.

L'ajustement de la largeur de raie $\Delta H(T)$ dans ces composés métalliques, comme la somme d'une contribution de type Korringa et d'un élargissement dû à l'échange (expression (145)), peut permettre d'obtenir les constantes $J$ et $J^{\prime}$, si les paramètres de champ cristallin et la constante $J_{\text {ex }}$ d'échange avec les électrons de conduction sont connus par des mesures indépendantes.

Signalons enfin que des études très semblables, mais avec un formalisme légèrement différent, ont été réalisées pàr Sugawara et al. [80].

c) Quant aux mesures du temps de relaxation longitudinal $T_{1}$ par les méthodes d'analyse en temps dans un système concentré en spins identiques, elles ne sont pas affectées par l'interaction d'échange isotrope, ni par la partie séculaire de l'interaction dipolaire, qui ne modifient pas la composante $m_{z}$ de l'aimantation totale : $\left[m_{\mathrm{z}}, \mathcal{H}_{\mathrm{ss}}^{\prime}\right]=0$.

Par contre, $\mathcal{H}_{\mathrm{ss}}^{\prime}$ contribue à établir l'équilibre au sein du système Zeeman par exemple après une " impulsion à $90^{\circ}$ ", en un temps de l'ordre de $T_{\mathrm{ss}} \sim T_{2}$, lorsque les niveaux Zeeman sont également espacés (présence du seul champ extérieur). Les processus non séculaires de l'interaction dipolaire (termes $C$, $D, E$ et $F$ ) ne conservant pas l'énergie globale, sont alors très peu probables.

6.2 LA RELAXATION "CROISÉE 》 (CROSS-RELAXATION). - Lorsque plusieurs transitions, d'énergies légèrement différentes, sont possibles au sein du système de spins identiques, ou bien entre deux espèces de spins différentes, apparaissent des processus de "relaxation croisée » [81] mettant en jeu un petit nombre d'ions, et dans lesquels l'énergie est " presque » conservée, le reliquat étant cédé au « bain spin-spin », ou fourni par ce dernier $\left({ }^{10}\right)$.

On commence par chercher à estimer la probabilité qu'un ion isolé (l'ion $i$ ) absorbe (ou cède) l'énergie $\hbar \omega_{0}$, sous l'influence par exemple du terme $S_{+i} S_{z j}$ de la partie non séculaire $(C)$ de $H_{\text {dip }}$, le quantum $\hbar \omega_{0}$ étant fourni (ou dissipé) par un réarrangement au sein du système dipolaire. Pour cela, on suppose que l'on peut appliquer la règle d'or de Fermi :

$$
W_{i}=\frac{1}{\hbar^{2}} \sum_{j}\left|\left\langle m_{j}, m_{i}+1|C| m_{j}, m_{i}\right\rangle\right|^{2} g(\omega=0) .
$$

La fonction $g(\omega)$ joue le rôle d'une densité spectrale de l'ensemble des spins, provenant de la partie séculaire $A+B$ de l'interaction spin-spin. La conservation

$\left({ }^{10}\right)$ Nous supposons ici que la relaxation spin-réseau du réservoir spin-spin est infiniment rapide. 
de l'énergie dans le système de spins (l'ion $i$ absorbe l'énergie $\hbar \omega_{0}$, le reste des spins fournissant $\hbar \omega_{0}$ ) implique la présence de $g(0)$ dans (150). La fonction $g(\omega)$ est centrée en $\omega_{0}$, et sa largeur $\Delta \omega$ peut être estimée par la méthode des moments si l'on suppose qu'elle est de forme gaussienne :

$$
g(\omega)=\frac{1}{\sqrt{2 \pi} \Delta \omega} \exp \left[-\frac{1}{2}\left(\frac{\omega-\omega_{0}}{\Delta \omega}\right)^{2}\right] .
$$

La probabilité $W_{i}$ de renversement du spin $i$ devient :

$$
\begin{array}{r}
W_{i}=\frac{1}{\hbar^{2}} C_{\mathrm{dip}}^{2} \frac{\left[S(S+1)-m_{i}\left(m_{i}+1\right)\right]}{\sqrt{2 \pi} \Delta \omega} \times \\
\times \exp \left[-\frac{1}{2}\left(\frac{\omega_{0}}{\Delta \omega}\right)^{2}\right] .
\end{array}
$$

$C_{\text {dip }}$, qui est une somme sur le réseau dipolaire, représente la « force " de la transition $\left(C_{\mathrm{dip}} \simeq g^{2} \mu_{\mathrm{B}}^{2} / r_{i j}^{3}\right)$.

On voit sur l'expression (152) que ce processus à un ion est d'autant plus probable que la largeur $\Delta \omega$ de la raie est importante (et proche de la fréquence de résonance $\left.\omega_{0}\right)$. Ce type de formule, qui ne représente qu'une approximation (parfois grossière), est à la base des calculs de probabilité de " relaxation croisée », et peut être utilisé pour décrire les probabilités de transition "à un ion" apparaissant dans le calcul des formes de raie Mössbauer en présence de relaxation spin-spin.

Par exemple, si l'on considère un ensemble d'ions identiques décrits par le hamiltonien de spin (expression (139)) :

$$
\mathscr{H}_{0}=-g \mu_{\mathrm{B}} H_{0} S_{\mathrm{z}}-D S_{\mathrm{z}}^{2} \quad(S=1),
$$

des transitions de relaxation croisée à deux ions, induites par le terme $S_{+i} S_{-j}$ de l'interaction d'échange ou dipolaire magnétique, peuvent avoir lieu avec une probabilité non négligeable si $\omega_{2} \simeq \omega_{1}$ (cf. Fig. 34). La petite différence d'énergie $\left|\omega_{2}-\omega_{1}\right|$ est fournie par le "bain spin-spin", et, si l'on suppose que les deux transitions ont la même largeur $\Delta \omega$, la probabilité d'un flip-flop entre les ions $\underline{i}$ et $\underline{j}$ peut s'écrire, de manière approchée [81] :

$$
W_{i j}^{\mathrm{RC}}=\frac{1}{\hbar^{2}} B_{\mathrm{dip}}^{2} \frac{1}{\sqrt{2 \pi} 2 \Delta \omega} \exp \left[-\left(\frac{\omega_{2}-\omega_{1}}{2 \Delta \omega}\right)^{2}\right],
$$

$$
\text { où } B_{\text {dip }} \text { est de l'ordre de } g^{2} \mu_{\mathrm{B}}^{2} / r_{i j}^{3} \text {. }
$$

La relaxation croisée a également été observée lorsque les ions sont couplés par paires par un terme d'échange isotrope, de constante J. En présence de champ magnétique, tel que : $g \mu_{\mathrm{B}} H_{0}>\mathfrak{J}$, les niveaux d'énergie d'un tel système de 2 ions identiques de spin $1 / 2$, sont déterminés par :

$$
\mathscr{H}_{0}=-g \mu_{\mathrm{B}} H_{0} S_{\mathrm{z}}+\mathcal{J} S_{\mathrm{z}}^{2},
$$

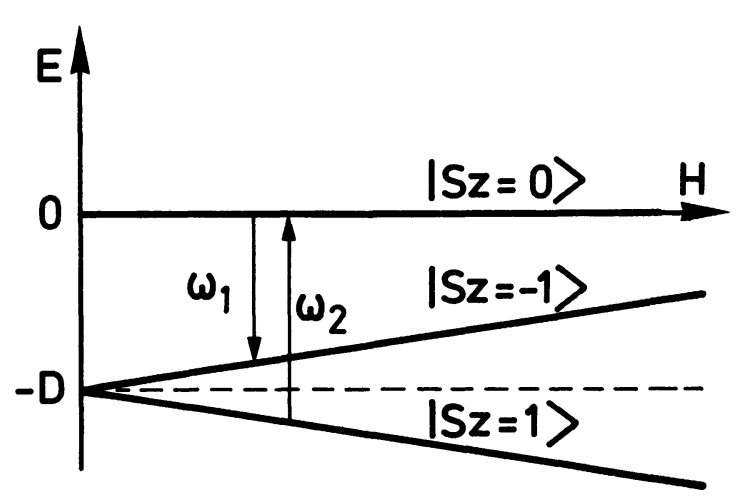

Fig. 34. - Niveaux d'énergie du hamiltonien de spin :

$$
\mathscr{H}_{0}=-g \mu_{\mathrm{B}} H S_{\mathrm{z}}-D S_{\mathrm{z}}^{2}
$$

pour $S=1$, en fonction du champ magnétique appliqué $H$.

[Energy levels of the spin-Hamiltonian :

$$
\mathscr{H}_{0}=-g \mu_{\mathrm{B}} H S_{\mathrm{z}}-D S_{\mathrm{z}}^{2}
$$

for $S=1$, as a function of the applied magnetic field $H$.]

où $S$ est le spin de la paire d'ions, qui peut valoir 0 ou 1 (cf. Fig. 35). Les transitions entre états sont dues à la partie non diagonale de l'échange, supposée avoir un spectre continu d'énergies propres [84]. Pour certaines valeurs " harmoniques » du champ magnétique $\left(H_{0}\right.$ et $H_{1}$ sur la Fig. 35), les différences d'énergie entre les diverses branches des courbes $E(H)$ sont dans un rapport rationnel $n / m$. Des transitions mettant en jeu $n+m$ paires d'ions peuvent alors conserver l'énergie (elles sont représentées par des flèches sur la Fig. 35). Les mesures de susceptibilité alternative sont bien adaptées à l'étude de ce type de relaxation croisée, car les temps caractéristiques $T_{21}$ de tels processus sont intermédiaires entre $T_{1}$ à basse température $\left(T_{1} \gtrsim 10^{-5} \mathrm{~s}\right)$ et $T_{2}\left(T_{2} \sim T_{\mathrm{ss}} \simeq 10^{-8}-10^{-9} \mathrm{~s}\right)$. Les courbes : $\chi^{\prime}=f(H)$ dans le domaine de fréquences autour du $\mathrm{MHz}$ montrent une «structure de résonance ", avec des maxima fortement marqués pour les valeurs harmoniques du champ magnétique, correspondant à un " accord » entre la fréquence $\omega$ de la sonde et la fréquence de relaxation croisée $1 / T_{21}$ $[21 b, c]$.

De nombreux calculs théoriques sur la dépendance en champ magnétique du temps de relaxation spinspin ont été effectués en relation avec les 'mesures de $\chi_{\|}(\omega)$ (voir par exemple les Réfs. [82-84]).

Enfin, la relaxation croisée entre espèces de spins différentes a été beaucoup étudiée dans le rubis $\mathrm{Al}_{2} \mathrm{O}_{3}$ : $\mathrm{Cr}^{3+}$, en présence d'impuretés d'autres ions de transition $\left(\mathrm{Cr}^{2+}, \mathrm{Mn}^{3+}, \mathrm{Ni}^{2+}\right)$ : citons les travaux récents et très complets sur la mesure de $1 / T_{1}$ de $\mathrm{Cr}^{3+}$ dans des monocristaux d'alumine [85], mettant en évidence une "structure de résonance" de $1 / T_{1}=f(\theta)$, où $\theta$ est l'angle entre le champ magnétique et l'axe cristallin, pour certaines valeurs de $\theta$ (angles "magiques») correspondant à des rapports simples entre les énergies 


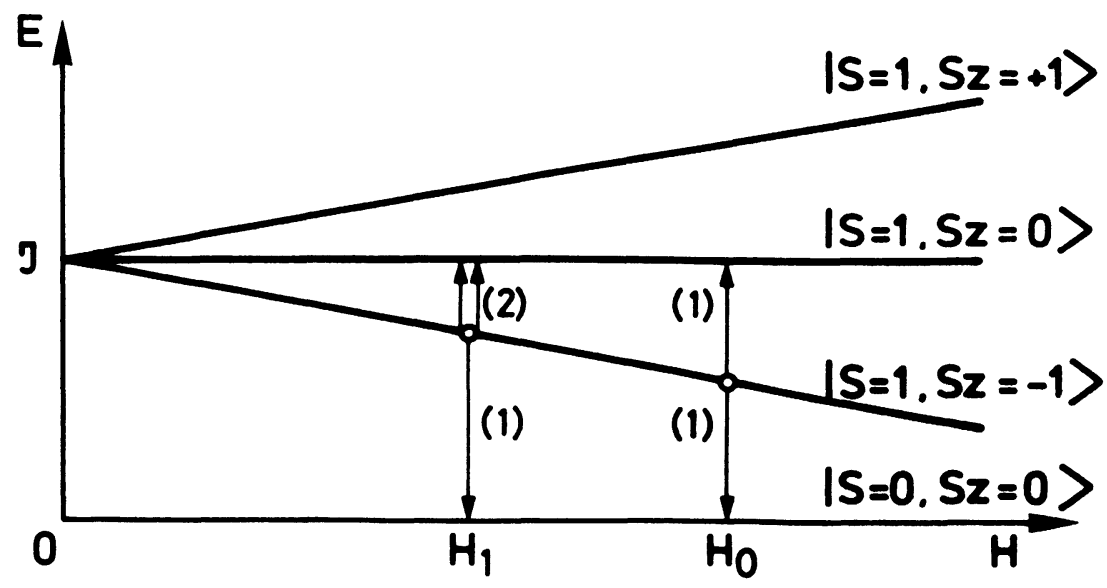

Fig. 35. - Niveaux d'énergie d'une paire d'ions $(S=1 / 2)$ couplés par l'échange (J) en fonction du champ magnétique; $H_{0}$ et $H_{1}$ sont deux valeurs " harmoniques » du champ appliqué $H$ (voir texte).

[Energy levels of a pair of ions $(S=1 / 2)$ coupled by exchange (J) as a function of the magnetic field; $H_{0}$ and $H_{1}$ are two " harmonic » values of the applied field $H$ (see text).]

de transitions des différents ions présents (les mesures sont réalisées à $T=4,2 \mathrm{~K}$ ).

6.3 LA RELAXATION SPIN-SPIN MESURÉE PAR EFFET MösSBAUER. - Les études de la relaxation spin-spin par effet Mössbauer possèdent la particularité de pouvoir être réalisées aussi bien dans la zone paramagnétique (sans champ magnétique extérieur) que dans la zone d'ordre magnétique. Elles concernent en majorité (comme d'ailleurs toutes les études de relaxation) les ions de Kramers : $\mathrm{Fe}^{3+}$ et les terres rares $\mathrm{Yb}^{3+}, \mathrm{Dy}^{3+}, \mathrm{Er}^{3+}$.

Signalons également quelques travaux sur la relaxation spin-spin de l'ion non-de Kramers $\mathrm{Tm}^{3+}[86$, 87]; ces résultats cependant se prêtent difficilement à l'interprétation, à cause de la complexité de la structure de champ cristallin de $\operatorname{Tm}^{3+}(J=6$, donc 13 niveaux).

Pour les ions de Kramers, dont l'état fondamental est un doublet magnétique, la fréquence de relaxation spin-spin à basse température possède la caractéristique d'être indépendante de la température. En effet, en zone paramagnétique sans champ appliqué, les transitions entre les deux états dégénérés du doublet, induites par $\mathfrak{H}_{\mathrm{ss}}$ mettent en jeu une énergie nulle (ou de -l'ordre de l'énergie hyperfine, négligeable lorsque $\left.k_{\mathrm{B}} T \gg \omega_{\mathrm{hpf}} \sim 0,1 \mathrm{~K}\right)$. Par contre, lorsqu'on élève la température, les doublets excités, qui deviennent appréciablement peuplés contribuent à la relaxation, et la fréquence de relaxation spin-spin peut montrer une variation en température. Cet effet est identique à celui décrit dans le paragraphe précédent ( $\$ 6.1$ ) pour la R.P.E. des ions $\mathrm{Er}^{3+}$ dans $\mathrm{LaBe}_{13}$. $\mathrm{La}$ raison pour laquelle, dans ce dernier système, on n'observe pas d'effets de relaxation spin-spin indépendants de la température tient au fait que la forme de raie R.P.E. à basse température n'est pas affectée par les «flip- flop " entre les deux états du doublet fondamental de $\mathrm{Er}^{3+}$.

Pour calculer la probabilité de transition "à un ion " induite par $\mathcal{H}_{\mathrm{ss}}$ entre les deux états d'un doublet dégénéré (ou quasi dégénéré), on peut s'inspirer de la méthode de la référence [81] ; ceci est possible lorsqu'il existe dans le cristal un axe de quantification " local " (axe de symétrie cristalline, champ magnétique appliqué ou moléculaire) que l'on choisit comme axe $\mathrm{O} z$. Dans ce cas, on peut décomposer $\varkappa_{\mathrm{ss}}$ en une partie $(A)$ n'induisant pas de transitions, mais conférant une certaine largeur $\Delta \omega_{\mathrm{ss}}$ au doublet, et une partie $(B+C+D+E+F)$ responsable de transitions à 2 ions $\left(S_{i}+S_{j-}, S_{i}-S_{j-}\right.$, etc...). La probabilité de transition s'écrit alors :

$$
W_{\mathrm{ss}}=\frac{1}{\hbar^{2}}|\mathfrak{B}|^{2} \rho(0)
$$

où : $\rho(0) \simeq \frac{1}{\sqrt{\pi} \Delta \omega_{\mathrm{ss}}}$ si l'on suppose que la « densité spectrale " $\rho(\omega)$ est gaussienne. La quantité $\mathfrak{B}$ représente l'élément de matrice de la partie non diagonale de $\mathcal{H}_{\mathrm{ss}}$ entre les deux états du doublet, ainsi qu'une somme sur les états quantiques possibles de l'autre ion mis en jeu $\left({ }^{11}\right)$.

La relaxation par les interactions dipolaires entre ions $\mathrm{Fe}^{3+}$ est discutée dans les références [41, 42 et 88]. Nous allons nous intéresser ici à la relaxation par échange dans les composés de terre rare, en distinguant les études réalisées en zone ordonnée et en zone paramagnétique.

( $\left.{ }^{11}\right)$ Remarquons que cette formule ne représente qu'une approximation, car, comme il a été dit au début de ce paragraphe, les états quantiques du système de spins sont déterminés par $\mathscr{H}_{0}+\mathscr{H}_{\mathrm{ss}}$, et non pas par $\mathscr{H}_{0}$ seul. 
6.3.1 Relaxation spin-spin en zone paramagnétique. Considérons un doublet de Kramers sur un site à symétrie axiale, de tenseur $\mathbf{g}: g_{\perp}, g_{\|}$. Dans le domaine de température : $k_{\mathrm{B}} T \ll \Delta_{\text {cc }}$ (énergie du premier doublet de champ cristallin), l'interaction d'échange (131) s'écrit en fonction du spin effectif $S=1 / 2$ des doublets :

$$
\mathscr{H}_{\mathrm{ech}}=\frac{1}{2}\left(g_{\mathrm{J}}-1\right)^{2} \sum_{i, j} \mathfrak{J}_{i j} \mathbf{J}_{i} \cdot \mathbf{J}_{j},
$$

d'où :

$$
\mathcal{H}_{\mathrm{ech}}=\frac{1}{2}\left(\frac{g_{\mathrm{J}}-1}{g_{\mathrm{J}}}\right)^{2} \sum_{i, j} \mathfrak{J}_{i j}\left(g \mathbf{S}_{i}\right)\left(\mathbf{g} \mathbf{S}_{j}\right) .
$$

Lorsque le doublet est fortement anisotrope $\left(g_{\|} \gg g_{\perp}\right)$, on peut quantifier les états électroniques suivant l'axe cristallin et les transitions "flip-flop » sont induites par la partie transverse de $\mathscr{H}_{\text {éch }}$ :

$\mathcal{H}_{\mathrm{ech}}^{\perp}=\frac{1}{4}\left(\frac{g_{\mathrm{J}}-1}{g_{\mathrm{J}}}\right)^{2} g_{\perp}^{2} \sum_{i, j} J_{i j}\left(S_{+i} S_{-j}+S_{+j} S_{-i}\right)$

La fréquence de relaxation $W_{\text {ss }}$ peut alors être évaluée à l'aide de l'expression (154) :

$$
W_{\mathrm{ss}}=\frac{1}{\hbar^{2}}|\mathfrak{B}|^{2} \rho(0)
$$

où :

$$
\Re \simeq \frac{1}{4}\left(\frac{g_{\mathrm{J}}-1}{g_{\mathrm{J}}}\right)^{2} g_{\perp}^{2} \bar{J}
$$

et

$$
\rho(0) \simeq \frac{1}{\sqrt{\pi} \Delta \omega_{\mathrm{ss}}} \simeq \frac{\hbar}{\sqrt{\pi} \frac{g_{\mathrm{J}}-1}{g_{\mathrm{J}}} g_{\mathrm{z}} \bar{J}}
$$

$\bar{\jmath}$ est ici une constante d'échange moyenne.

On a donc, pour un ensemble de doublets de Kramers sur des sites équivalents :

$$
W_{\mathrm{ss}} \simeq \frac{1}{16 \hbar}\left(\frac{g_{\mathrm{J}}-1}{g_{\mathrm{J}}}\right)^{3} \frac{g_{\perp}^{4}}{g_{\mathrm{z}}} \overline{\mathrm{J}}
$$

La fréquence de relaxation spin-spin est indépendante de la température, et vaut par exemple, pour $\vec{J}=10 \mathrm{~K}$, environ : $10^{11} \mathrm{~Hz}$.

Un tel calcul n'est pas justifié lorsque la symétrie du site est cubique $\left(g_{\perp}=g_{\|}\right)$: en effet, il n'y a pas d'axe de quantification microscopique en l'absence de champ magnétique, et tous les termes de $\mathscr{H}_{\mathrm{ech}}$ peuvent induire des transitions de relaxation. Cette situation a été rencontrée dans l'étude de la relaxation (induite par les interactions dipolaires) de $\mathrm{Yb}^{3+}$ dans le composé cubique $\mathrm{Cs}_{2} \mathrm{NaYbCl}_{6}$ [89]. Les auteurs ont alors utilisé une formule semi-classique dans l'approximation du spectre blanc $\left(\omega_{\text {hpf }} \ll 1 / \tau_{\text {c }}\right.$, cf. expressions (7) et (8)) pour estimer la fréquence de relaxation :

$$
W_{\mathrm{ss}} \sim g^{2} \mu_{\mathrm{B}}^{2}\left\langle H_{\mathrm{dip}}^{2}\right\rangle \tau_{\mathrm{c}},
$$

où $\left\langle H_{\mathrm{dip}}^{2}\right\rangle$ est la valeur quadratique moyenne du champ dipolaire, qui est considéré, ainsi que $\tau_{\mathrm{c}}$, comme un paramètre ajustable dans l'interprétation des spectres Mössbauer (la valeur obtenue pour $\tau_{c}$ a d'ailleurs montré que : $1 / \tau_{\mathrm{c}} \sim \omega_{\mathrm{hpf}}$, c'est-à-dire que l'approximation du spectre blanc n'est pas valable dans ce cas).

Lorsque le doublet de Kramers est extrêmement anisotrope $\left(g_{\perp} \ll g_{z}\right)$, comme par exemple lorsque l'ion $\mathrm{Dy}^{3+}$ ou $\mathrm{Er}^{3+}$, en symétrie axiale, possède un doublet fondamental de champ cristallin $\left|J_{z}= \pm 15 / 2\right\rangle$, l'expression (157) montre que la relaxation spin-spin peut être lente $\left(W_{\mathrm{ss}} \ll \omega_{\mathrm{hpf}} \sim 10^{9} \mathrm{~Hz}\right)$ [90].

Une exception à cette règle a été rencontrée dans le composé perovskite $\mathrm{YbAlO}_{3}$ [91] : l'ion $\mathrm{Yb}^{3+}$ y possède un tenseur $\mathbf{g}$ extrêmement anisotrope (fondamental $\left.\sim\left|J_{z}= \pm 7 / 2\right\rangle\right)$, mais il existe dans ce composé deux types de sites non équivalents, ayant des axes de facile aimantation faisant entre eux un angle $\alpha$ de $60^{\circ}$. Le calcul de l'interaction d'échange à l'aide de (155) fait alors apparaître des termes croisés $g_{-} g_{z} \sin \alpha S_{i z} S_{j+}$ qui donnent la contribution dominante à la fréquence de relaxation, qui est de l'ordre de $5 \times 10^{10} \mathrm{~Hz}$.

Enfin, la relaxation d'une impureté de $\mathrm{Yb}^{3+}$ dans les perovskites. $\mathrm{TRAlO}_{3}(\mathrm{TR}=$ terre rare $)$ [92] révèle des caractéristiques variées selon la nature de l'ion de la matrice : relaxation dipolaire pour $\mathrm{Yb}^{3+}$ dans $\mathrm{DyAlO}_{3}$, relaxation par échange Yb-TR dans $\mathrm{ErAlO}_{3}$ et $\mathrm{GdAlO}_{3}$, fluctuations très « lentes » dans les matrices $\mathrm{TbAlO}_{3}$ et $\mathrm{HoAlO}_{3}$, où l'ion de Van Vleck $\mathrm{Tb}^{3+}$ ou $\mathrm{Ho}^{3+}$ possède un état singulet de champ cristallin fondamental. Dans le composé $\mathrm{HoAlO}_{3}$, la présence proche du premier singulet excité $(\Delta \simeq 8 \mathrm{~K})$ conduit à une dépendance thermique de la fréquence de relaxation par échange, due au peuplement progressif de l'état excité.

6.3.2 Fluctuations des moments électroniques en zone ordonnée. - Considérons un doublet de Kramers soumis à un champ moléculaire $H_{m}$. L'écart entre les deux états : $\Delta(T)=g \mu_{\mathrm{B}} H_{m}(T)$ est une fonction décroissante de la température, et s'annule à la température critique. A très basse température $\left(k_{\mathrm{B}} T \ll \Delta(T)\right)$, seul le niveau fondamental est peuplé, et le spectre Mössbauer observé montre un champ hyperfin $H_{\mathrm{hpf}}$ correspondant à cet état électronique. L'écart entre les raies est : $\omega_{\mathrm{hpf}}=g_{\mathrm{n}} \mu_{\mathrm{n}} H_{\mathrm{hpf}}$. Lorsqu'on augmente la température, la population du niveau excité augmente, tandis que $\Delta(T)$ diminue. L'aspect du spectre Mössbauer va alors dépendre de manière critique de la fréquence de relaxation $W_{\text {ss }}$ entre les deux états, induite par l'interaction d'échange, que nous supposerons seule en jeu à basse température. Si la relaxation est « lente » $\left(W_{\text {ss }} \ll \omega_{\text {hpf }}\right)$ dans la zone ordonnée, on prévoit une superposition statique des spectres hyperfins identiques provenant des deux états du doublet, pondérés par leur facteur de Boltzmann. On ne doit donc observer aucun changement du spectre Mössbauer lorsqu'on augmente la température. 
$\mathrm{Au}$ contraire, si la relaxation est "rapide" $\left(W_{\mathrm{ss}} \gg \omega_{\mathrm{hpf}}\right)$, le champ hyperfin moyen vu par le noyau vaut :

$$
\left\langle H_{\mathrm{hpf}}\right\rangle_{\mathrm{T}}=H_{\mathrm{hpf}} \operatorname{tgth} \frac{\Delta(T)}{2 k_{\mathrm{B}} T},
$$

lorsqu'on peut considérer que les deux états restent conjugués de Kramers dans le champ d'échange $\left(\Delta(T) \ll \Delta_{\mathrm{cc}}\right)$. Le champ hyperfin mesuré sur le spectre décroît lorsque la température augmente, pour s'annuler à $T=T_{\mathrm{c}}$.

Dans la zone d'ordre magnétique, l'axe de quantification local est déterminé par le champ moléculaire, et la relaxation est induite par le terme non diagonal de $\mathcal{H}_{\text {ech }}$ (expression (156)), lorsque la température n'est pas trop basse devant $T_{\mathrm{c}}$ (lorsque $T \ll T_{\mathrm{c}}$, le niveau fondamental est seul peuplé, et la relaxation est négligeable). L'efficacité de la relaxation par l'échange, qui obéit à la règle de sélection $\Delta J_{z}= \pm 1$, dépend des fonctions d'onde électroniques, qui sont déterminées par la compétition entre l'interaction de champ moléculaire et le champ cristallin.

En règle générale, les états quantiques dans la zone ordonnée sont des combinaisons linéaires d'états $\left|J_{\mathrm{z}}\right\rangle$ (ou $\left|S_{\mathrm{z}}\right\rangle$ pour les ions $S$ ), et la fréquence de relaxation par échange est du même ordre de grandeur que $W_{\text {ss }}$ en zone paramagnétique, donnée par l'expression (157).

Par exemple, dans les composés ferriques ordonnés, les états propres sont les états Zeeman $\left|S_{\mathrm{z}}=m\right\rangle$, d'énergie : $E_{m}=-g \mu_{\mathrm{B}} m H_{m}$, le champ cristallin de $\mathrm{Fe}^{3+}$ (ion $\mathrm{S}$ ) étant très faible. La relaxation par échange est alors " rapide" $\left(W_{\mathrm{ss}} \gg \omega_{\mathrm{hpf}} \simeq 10^{8} \mathrm{~Hz}\right)$. Pour les composés de terre rare, dont la "fenêtre" Mössbauer est centrée autour de $10^{9} \mathrm{~Hz}$, la relaxation en zone ordonnée est en général "rapide", et dans certains cas est mesurable $\left(W_{\mathrm{ss}} \gtrsim \omega_{\mathrm{hpf}}\right)$.

La première observation des effets de la relaxation en zone d'ordre magnétique a été réalisée par $\mathrm{W}$. Wiedemann et al. [93] dans le composé $\mathrm{ErFeO}_{3}$, et interprétée à l'aide d'un modèle stochastique simple par Nowik et al. [94]. Dans ce modèle, la forme du spectre Mössbauer est fonction du rapport : $W_{\mathrm{ss}} / \Delta(T)$, où $\Delta(T)$ est l'écart de champ moléculaire. Lorsque $\Delta(T)$ est connu (par des mesures optiques par exemple), on peut alors mesurer $W_{\mathrm{ss}}$. Sinon, on peut faire l'hypothèse que $W_{\mathrm{ss}}$ est constant dans la zone ordonnée, et égal à la fréquence mesurée en zone paramagnétique juste au-dessus de $T_{c}$. Différentes études sur les composés d'erbium [95], de dysprosium [96], et d'ytterbium [97] en zone ordonnée ont ainsi mesuré des fréquences de relaxation spin-spin de l'ordre de $10^{10}-10^{11} \mathrm{~Hz}$. Plus récemment, la relaxation par échange de l'impureté $\mathrm{Yb}^{3+}$ dans $\mathrm{GdAlO}_{3}$ a été observée dans la zone ordonnée [92]. Les spectres, ajustés à l'aide du modèle de la référence [90], sont représentés sur la figure 36 : remarquons que la variation de la forme du spectre, qui dépend de $W_{\mathrm{ss}} / \Delta$, est due principalement à la variation thermique du champ

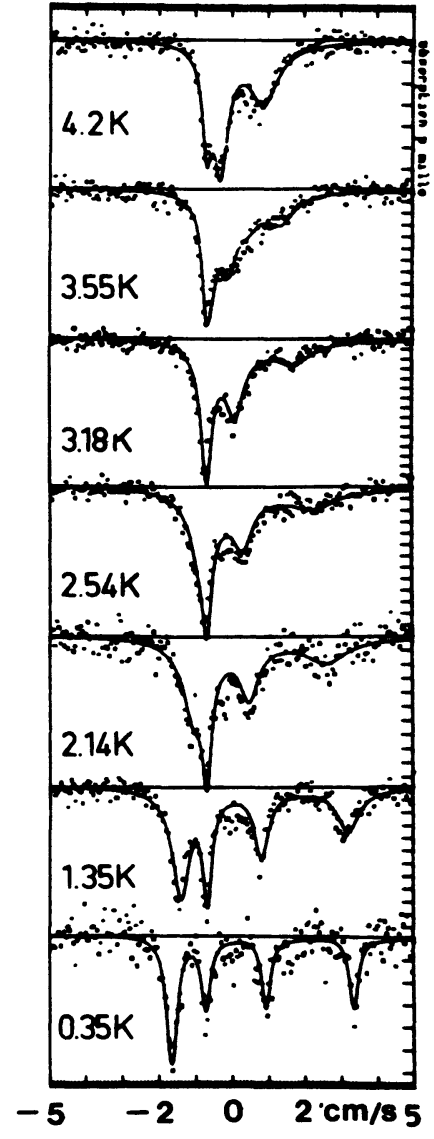

Fig. 36. - (D'après [93]) Spectres Mössbauer d'absorption de ${ }^{170} \mathrm{Yb}^{3+}(2,5 \%$ at. $)$ dans $\mathrm{GdAlO}_{3}$, dans la zone paramagnétique $(T=4,2 \mathrm{~K})$ et dans la zone d'ordre antiferromagnétique des ions $\mathrm{Gd}^{3+}\left(T_{\mathrm{N}}=3,87 \mathrm{~K}\right)$.

[(After [93]) Absorption Mössbauer spectra of ${ }^{170} \mathrm{Yb}^{3+}$ $(2.5 \%$ at. $)$ in $\mathrm{GdAlO}_{3}$, in the paramagnetic zone $(T=4.2 \mathrm{~K})$ and in the zone of antiferromagnetic ordering of the $\mathrm{Gd}^{3+}$ ions $\left(T_{\mathrm{N}}=3.87 \mathrm{~K}\right)$.]

moléculaire $\left(\Delta(T)=g \mu_{\mathrm{B}} H_{m}(T)\right)$ et non à celle de $W_{\mathrm{ss}}$, qui est pratiquement constant et égal à : $W_{\mathrm{ss}} \simeq 0,7 \times$ $10^{10} \mathrm{~Hz}$.

Dans certains cas, l'interaction de champ moléculaire ne modifie pas sensiblement les fonctions d'onde du doublet fondamental de champ cristallin. Si celui-ci est extrêmement anisotrope $\left(g_{\perp} \ll g_{z}\right)$, la relaxation spin-spin, comme nous l'avons vu, est inefficace; cette situation est observée dans le grenat DyAlG [98], ainsi que dans d'autres composés de dysprosium et d'erbium, où le passage de la température critique $T_{\mathrm{c}}$ n'est accompagné d'aucune variation du spectre Mössbauer.

\section{Conclusion.}

Les travaux consacrés à la relaxation paramagnétique dans les solides se sont remarquablement développés depuis une quinzaine d'années. D'une part, à côté des techniques «classiques » (R.P.E., effet Mössbauer), 
on a utilisé des méthodes certainement plus difficiles à mettre en œuvre dans les solides : R.M.N. sur le noyau de l'impureté ou sur un noyau voisin, mesures de corrélations $\gamma-\gamma$ différentielles à la suite d'une réaction nucléaire de transmutation sur un noyau de la matrice, mesure de la section efficace de diffusion magnétique des neutrons lents, qu'il faut soigneusement séparer de toutes les autres contributions (diffusion par les phonons, diffusion élastique). D'autre part, l'outillage théorique nécessaire pour interpréter les spectres en présence de relaxation croît sans cesse en sophistication, comme en témoigne l'article joint de HartmannBoutron [42] sur le calcul des formes de raie en effet Mössbauer.

Ces progrès, dans le domaine tant expérimental que théorique, ont permis une meilleure compréhension des mécanismes microscopiques régissant les échanges d'énergie dans les solides, et une estimation quantitative de divers paramètres physiques tels que les énergies de champ cristallin, les constantes de l'échange interionique et de l'échange entre électrons localisés et itinérants.
Enfin, faute de place, de nombreuses applications importantes des études de relaxation ont été passées sous silence dans cet article. Citons les mesures au voisinage des points critiques des transitions magnétiques (fluctuations critiques), le superparamagnétisme des grains fins, le problème des phénomènes dynamiques dans les " verres de spin » en-dessous de la température de gel, l'étude des fluctuations rapides des ions de transition et de quelques ions de terre rare (Ce, Eu, Sm, Yb) dans les composés Kondo ou à valence intermédiaire, l'observation d'états métastables et de leur relaxation à l'aide de la spectroscopie Mössbauer d'émission, sujet décrit de manière très complète dans l'article joint d'Imbert [38].

\section{Remerciements.}

Je remercie F. Hartmann-Boutron pour de nombreuses et fructueuses discussions sur le problème de la relaxation, et $\mathbf{M}$. Goldman pour ses remarques critiques concernant le manuscrit.

\section{Bibliographie}

[1] Abragam, A., Les principes du magnétisme nucléaire (P.U.F.) 1961.

[2] Gordon, R. G., in Adv. Magn. Reson. 3, ed. J. S. Waugh (Academic Press) 1968, p. 1.

[3] Voir Topics in Current Physics 3, Eds. Lovesey et Springer, 1976.

[4] Abragam, A. et Pound, R. V., Phys. Rev. 92 (1953) 943.

[5] Redfield, A. G., in Adv. Magn. Reson. 1, ed. J. S. Waugh (Academic Press) et références citées, 1965, p. 1.

[6] Hartmann-Boutron, F. et Spanjaard, D., J. Physique 36 (1975) 307.

[7] Scott, P. L. and JefFries, C. D., Phys. Rev. 127 (1962) 32.

[8] Hasegawa, H., Progr. Theor. Phys. 21 (1959) 483.

[9] Spin-lattice Relaxation in lonic Solids, Ed. A. A. Manenkov, R. Orbach (Harper and Row), 1966.

[10] ORBaCH, R. in Proceedings of the 14th Conference on Low. Temp. Physics (LT14) 5 (1975) 375.

[11] Voir par exemple : KoNDo, J., Solid State Phys. 23 (1969) 183

[12] Hartmann-Boutron, F., I, publication jointe.

[13] Blatt, J. M. et WeIsSKopf, V. F., in Theoretical Nuclear Physics (New York) 1952

[14] Voir par exemple : Blume, M., in Hyperfine Structure and Nuclear Radiation (North-Holland, Amsterdam) eds. Matthias and Shirley, 1968, p. 911.

[15] Pour plus de détails, voir : Hartmann-Boutron, F., Ann. Phys. 9 (1975) 285.

[16] GoRTer, C. J., Paramagnetic Relaxation (Amsterdam) 1947.

[17] Cooke, A. H., Rep. Prog. Phys. 13 (1950) 276.

[18] GORTER, C. J., in Fluctuation, Relaxation and Resonance in Magnetic Systems ed. D. Ter Haar (Oliver and Boyd) 1961, p. 87.

[19] Gorter, C. J., 'in Prog. Low Temp. Phys. 2, ed. C. J. Gorter, p. 266.
[20] Par exemple, Eukelhof, H. M. C., Pouw, C. L. M., VAn DuYneveldt, A. J., Physica 62 (1972) 257.

[21] Voir par exemple :

a) Smits, L. J., Derksen, H. E., Verstelle, J. C., GORTER, C. J., Physica 22 (1956) 773.

b) Von Klot, R. et Weber, G., Z. Phys. 209 (1968) 380.

c) Kötzler, J., Physica 60 (1972) 375.

d) Grambow, I., Z. Physik 257 (1972) 245.

[22] Miyako, Y., Chikazawa, S., Saito, T., Yuochunàs, Y. G., J. Phys. Soc. Japan 48 (1980) 329.

[23] Mulder, C. A. M., Van DuYneVeld , A. J., Mydosh, J. A., Phys. Rev. B 23 (1981) 1384.

[24] Standley, K. J., VAughan, R. A., Electron spin relaxation phenomena in solids (London) 1969.

[25] Geschwind, S., in Electron Paramagnetic Resonance, ed. S. Geschwind (Plenum Press) 1972.

[26] Baberschke, K. et Tsang, E., Phys. Rev. Lett. 45 (1980) 1512.

[27] Voir par exemple : Davidov, D., Retrori, C., Dixon, A., BaberschKe, K., ChOCK, E. P. et Orbach, R., Phys. Rev. B 8 (1973) 3563.

[28] Bloch, F., Phys. Rev. 70 (1946) 460.

[29] Hirst, L. L., Phys. Rev. 181 (1969) 597.

[30] Kubo, R., Rep. Prog. Phys. 29 (1966) 255.

[31] Marshall, W. et Lowde, R. D., Rep. Prog. Phys. 31 (1968) 705.

[32] LöWenhaupt, Mr Holland-Moritz, E., J. Magn. Magn. Mat. 14 (1979) 227.

[33] a) Pierre, J., Murani, A. P., Galéra, R. M., J. Phys. F 11 (1981) 679.

b) Pierre, J. et Murani, A., in C.E.F. and structural effects in $4 f$-electron systems, eds. J. E. Crow, R. P. Guertin, T. W. Mihalisin (Plenum Press) 1981.

c) Holland-Moritz, E., Loewenhaupt, M., Schmatz, W., Wohlleben, D. K., Phys. Rev. Lett. 38 (1977) 983. 
[34] Callen, H. B., in Fluctuation, Relaxation and Resonance in Magnetic Systems, ed. D. Ter Haar (Oliver and Boyd) 1961.

[35] Voir par exemple : Weber, M. J., Solid State Commun. 12 (1973) 741.

[36] Yen, W. M., Scott, W. C., Schawlow, A. L., Phys. Rev. 136A (1964) 271;

Flach, R., Hamilton, D. S., Selzer, P. M., Yen, W. M., Phys. Rev. B 15 (1977) 1248.

[37] HüFNER, S., Optical Spectra of Transparent Rare Earth Compounds, chap. 4 (Academic Press) 1978.

[38] Imbert, P., Publication jointe.

[39] Voir par exemple : FurRer, A., KJEMS, J., VoGt, O., J. Phys. C 5 (1972) 2246.

[40] Wickman, H. H., in Hyperfine Structure and Nuclear Radiation (North-Holland, Amsterdam) eds. Matthias and Shirley, 1968, p. 928.

[41] a) Bhargava, S. G., Knudsen, J. E., MфRUP, S., J. Phys. C 12 (1979) 2879.

b) MфRUP, S., Paramagnetic and Superparamagnetic Relaxation Phenomena studied by Mössbauer Spectroscopy (Polyteknisk forlag) (Danemark) 1981.

[42] HartmanN-Boutron, F., II, publication jointe.

[43] a) Hirst, L. L., J. Phys. Chem. Solids 31 (1970) 655.

b) Gonzalez-Jimenez, F., Imbert, P., HartmanNBoutron, F., Phys. Rev. B 9 (1974) 95.

[44] Levy, R. M. and Shirley, D. A., Phys. Rev. 140 (1965) B 811.

[45] a) Barth, H. J., Nishiyama, K., Riegel, D., Phys. Lett. 77A (1980) 365.

b) Barth, H. J., Netz, G., Nishiyama, K., Riegel, D., Phys. Rev. Lett. 45 (1980) 1015.

c) Luszik-Bhadra, M., Barth, H. J., BROCKSCH, H. J., Netz, G., Riegel, D., Bertschat, H. H., Phys. Rev. Lett. 47 (1981) 871.

[46] Pour une revue, voir Narath, A., Physica Scripta 11 (1975) 237.

[47] Mms, W. B., in Electron Paramagnetic Resonance, ed. S. Geschwind (Plenum Press) 1972.

[48] Alloul, H., Physica 86-88B (1977) 449.

[49] Alloul, H., HipPert, F., IsHII, H., J. Phys. F 9 (1979) 725.

[50] Follstaedt, D., Narath, A., Phys. Rev. B 19 (1979) 1374.

[51] Anderson, P. W., J. Phys. Soc. Japan 9 (1954) 316.

[52] Anderson, P. W. et Weiss, P. R., Rev. Mod. Phys. 25 (1953) 269.

[53] Kuво, R., in Fluctuation, Relaxation and Resonance in Magnetic Systems, ed. D. Ter Haar (Oliver and Boyd) 1961.

[54] Moret, J. M., Orbach, R., Peter, M., Shaltiel, D., Süss, J. T., ZINGG, W., Devine, R. A. B., ZIMMERMANN, P. H., Phys. Rev. B 11 (1974) 2002.

[55] Chui, R., Orbach, R., Gehman, B. L., Phys. Rev. B 2 (1970) 2298.

[56] TaO, L. J., Davidov, D., Orbach, R., Chock, E. P., Phys. Rev. B 4 (1971) 5;

Chock, E. P., Davidov, D., ORbaCh, R., Rettori, C., TAO, L. J., Phys. Rev. B 5 (1972) 2735.

[57] Barnes, S. E., Dupraz, J., Orbach, R., J. Appl. Phys. 42 (1971) 1659.

[58] OrbaCH, R., J. Magn. Magn. Mat. 15-18 (1980) 706.

[59] Walker, M. B., Phys. Rev. B 1 (1970) 3690.

[60] PlefKa, T., Phys. Status Solidi (b) 55 (1973) 129.

[61] Barnes, S. E., Phys. Rev. B 9 (1974) 4789.
[62] Becker, K. W., Fulde, P., Keller, J., Z. Physik B 28 (1977) 9.

[63] Dattagupta, S., Hyperfine Interactions 11 (1981) 77.

[64] Mori, H., Progr. Theor. Phys. 34 (1965) 399.

[65] Stevens, K. W. H., Rep. Progr. Phys. 30 (1967) 189.

[66] Orbach, R. et Stapleton, H. J., in Electron Paramagnetic Resonance, ed. S. Geschwind, 1972.

[67] Bonville, P., Garcin, C., Gérard, A., Imbert, P., Jéhanno, G., Phys. Rev. B 23 (1981) 4293.

[68] Ruby, R. H., Benoit, H., JefFries, C. D., Phys. Rev. 127 (1962) 51.

[69] Roger, G., More, C., Blanc, C., J. Physique 41 (1980) 169.

[70] Orbach, R., Blume, M., Phys. Rev. Lett. 8 (1962) 478.

[71] van Der Bilt, A., van DuYneveldt, A. J., Physica 100B (1980) 324.

[72] Blanchard, C., Gaillard, B., Deville, A., J. Physique 40 (1979) 1179.

[73] Korringa, J., Physica 16 (1950) 601.

[74] Barberis, G. E., Davidov, D., Donoso, J. P., RetTori, C., Suassuna, J. F., Dokter, H. D., Phys. Rev. B 19 (1979) 5495.

[75] Kittel, C. et Abrahams, E., Phys. Rev. 90 (1953) 238.

[76] McMillan, M. et Opechowski, W., Can. J. Phys. 38 (1960) 1168.

[77] Bloch, J. M., Davidov, D., Dokter, H. D., Physica $101 B$ (1980) 189.

[78] a) Davidov, D., Zevin, V., Levin, R., Shaltiel, D., BaberschKe, K., Phys. Rev. B 15 (1977) 2771.

b) Davidov, D., Barberis, G., Rettori, C., Suassuna, J. F., Levin, R., Solid State Commun. 31 (1979) 867.

c) Levin, R., Davidov, D., Grayevsky, A., Shaltiel, D., Zevin, V., J. Phys. F 10 (1980) 1285.

[79] Zevin, V., Davidov, D., Levin, R., Shaltiel, D., BaberschKe, K., J. Phys. F 7 (1977) 2193.

[80] Sugawara, K., Huang, C. Y., CoOper, B. R., Phys. Rev. B 11 (1975) 4455;

Sugawara, K., J. Phys. Soc. Japan 42 (1977) 1154.

[81] Bloembergen, N., Shapiro, S., Pershan, P. S., Artman, J. O., Phys. Rev. 114 (1959) 445.

[82] Tuon, J. A., Physica 30 (1964)1.

[83] Sauermann, G., Physica 32 (1966) 2017.

[84] Sauermann, G., Schwegler, H., Z. Physik 209 (1968) 355.

[85] Bates, C. A., Steggles, P., Gavaix, A., Vasson, A. M., Vasson, A., J. Physique 39 (1978) 315;

Bates, C. A., Steggles, P., Vasson, A., Vasson, A. M., J. Physique 40 (1979) 977.

[86] Clauser, M. J., Mössbauer, R. L., Phys. Rev. 178 (1969) 559.

[87] Dixon, N. S., Fritz, L. S., Mahmud, Y., Triplett, B. B., Hanna, S. S., von Eynatten, G., J. Physique Colloq. 41 (1980) C1-25.

[88] Wegener, H., Wimmer, K., Seyboth, D., Zeman, N., Hyp. Int. 12 (1982) 15.

[89] Shenoy, G. K., Dunlap, B. D., Dattagupta, S., As.CH, L., Phys. Rev. Lett. 37 (1976) 539.

[90] NowIK, J., Phys. Lett. 15 (1965) 219.

[91] Bonville, P., Hodges, J. A., Imbert, P., HartmanNBoutron, F., Phys. Rev. B 18 (1978) 2196.

[92] Bonville, P., Hodges, J. A., Imbert, P., J. Physique 41 (1980) 1213.

[93] Wiedemann, W., Zinn, W., Z. Angew. Phys. 20 (1966) 327. 
[94] Nowik, I., Wickman, H. H., Phys. Rev. Lett. 17 (1966) 949.

[95] Zinn, W. et Wiedemann, W., J. Appl. Phys. 39 (1968) 839 ;

Eibschütz, M., Cohen, R. L., West, K. W., Phys. Rev. 178 (1969) 572.

[96] Khurgin, B., Nowik, I., Rakavy, M., OFer, S., J. Phys. Chem. Solids 31 (1970) 49 et références citées.

[97] OFER, S., NowIK, I., Physics Lett. 24A (1967) 88;

Nowik, I., Ofer, S., Wernick, J. H., Physics Lett. 24A (1967) 89.

[98] Nowik, I., Wickman, H. H., Phys. Rev. 140 (1965) A869. 